TRANSACTIONS OF THE

AMERICAN MATHEMATICAL SOCIETY

Volume 362, Number 2, February 2010, Pages 1047-1096

S 0002-9947(09)04896-X

Article electronically published on September 9, 2009

\title{
FORWARD-BACKWARD STOCHASTIC DIFFERENTIAL EQUATIONS WITH MIXED INITIAL-TERMINAL CONDITIONS
}

\author{
JIONGMIN YONG
}

\begin{abstract}
Well-posedness of forward-backward stochastic differential equations (FBSDEs, for short) in $L^{p}$ spaces with mixed initial-terminal conditions is studied. A notion of Lyapunov operator is introduced, whose existence leads to a priori estimates of the adapted solutions sufficient for the well-posedness of the corresponding FBSDEs, via the method of continuation. Various situations are discussed under which Lyapunov operators do exist.
\end{abstract}

\section{INTRODUCTION}

Throughout this paper, we let $(\Omega, \mathcal{F}, \mathbb{F}, \mathbb{P})$ be a complete filtered probability space, on which a standard one-dimensional Brownian motion $W(\cdot)$ is defined, with $\mathbb{F} \equiv\left\{\mathcal{F}_{t}\right\}_{t>0}$ being its natural filtration augmented by all the $\mathbb{P}$-null sets. We consider the following system of stochastic differential equations:

$$
\left\{\begin{array}{l}
d X(t)=b(t, X(t), Y(t), Z(t)) d t+\sigma(t, X(t), Y(t), Z(t)) d W(t) \\
d Y(t)=g(t, X(t), Y(t), Z(t)) d t+Z(t) d W(t) \\
X(0)=\gamma(X(T), Y(0)), \quad Y(T)=h(X(T), Y(0))
\end{array}\right.
$$

where $b, \sigma, g, \gamma$, and $h$ are suitable maps. The above is referred to as a forwardbackward stochastic differential equation (FBSDE, for short; see [10]) with mixed initial-terminal conditions. Roughly speaking, in the above, the first equation with the first condition in the third line is an initial value problem of an SDE (with $Y(\cdot)$ and $Z(\cdot)$ being "parameter processes"), and the second equation with the second condition in the third line is a terminal value problem for an SDE (with $X(\cdot)$ being a "parameter process"), which by now is known to be a backward stochastic differential equation (BSDE, for short). We may refer to the first two equations as a forward equation and a backward equation, respectively, and to the last two equalities as mixed initial-terminal conditions. The 5 -tuple $(b, \sigma, g, \gamma, h)$ is called the generator of FBSDE (1.1). A triple of $\mathbb{F}$-adapted processes $(X(\cdot), Y(\cdot), Z(\cdot))$ having certain properties (see below for precise definitions) is called an adapted

Received by the editors December 28, 2007 and, in revised form, August 1, 2008.

2000 Mathematics Subject Classification. Primary 60H10.

Key words and phrases. Forward-backward stochastic differential equations, mixed initialterminal conditions, Lyapunov operator, method of continuation.

This work was supported in part by the NSF grant DMS-0604309.

(C)2009 American Mathematical Society Reverts to public domain 28 years from publication 
solution to (1.1) if it satisfies (1.1) in the usual Itô sense. Recall that FBSDEs with the following initial-terminal conditions:

$$
X(0)=X_{0}, \quad Y(T)=h(X(T)),
$$

are well studied (see [2], 19], 18], 14, 28], 23], 20], 21], 15], 13], 7], 31, for examples). For convenience, we refer to this as the classical case. The new feature in (1.1) is that both $X(0)$ and $Y(T)$ depend on $(X(T), Y(0))$. Therefore, (1.1) is a natural (mathematical) extension of classical FBSDEs. We will present a couple of more interesting motivations leading to FBSDE (1.1) in the next section. It is known that, due to the nature of the equation, even if all the functions involved are smooth with bounded derivatives in $(X, Y, Z)$ (which leads to the usual uniform Lipschitz conditions), FBSDE (1.1) does not necessarily have an adapted solution (even for the classical case, see [20]). We point out that the study of general nonlinear boundary-value problems for ordinary differential equations is quite mature; see [24, 6], and the extensive references cited therein.

The method that will be used in the current paper is the so-called method of continuity. Roughly speaking, we introduce a family of FBSDEs parameterized by $\rho \in[0,1]$, denoted by $\mathcal{E}(\rho)$ for convenience, so that $\mathcal{E}(1)$ coincides with (1.1) and $\mathcal{E}(0)$ is always well-posed. Moreover, under certain conditions, one can show that there exists an $\varepsilon>0$ such that as long as $\mathcal{E}(\rho)$ is well posed, so is $\mathcal{E}((\rho+\varepsilon) \wedge 1)$. Then, inductively, we obtain the well-posedness of FBSDE (1.1). A key in such a procedure is to establish a uniform a priori estimate for possible adapted solutions to $\mathcal{E}(\rho)$. To this end, certain kinds of compatibility conditions on the generator $(b, \sigma, g, \gamma, h)$ have to be assumed (see [14, 28], 23], 21], for classical cases). In this paper, inspired by [28, we introduce the notion of Lyapunov operator for the considered FBSDE (1.1), which is a solution to a suitable Riccati type differential inequality with constraints. The existence of a Lyapunov operator will lead to $a$ priori estimates needed for establishing the well-posedness of the FBSDEs in $L^{2}$ spaces. With some additional effort we will refine the estimates so that the $L^{p}$ well-posedness (with $p \geq 2$ ) for the FBSDE (1.1) can be obtained. We would like to point out that the $L^{p}$-estimate for adapted solutions (with $p>2$ ) will play a crucial role in studying necessary conditions for optimal controls of FBSDEs with spike variations of controls. Some careful study along this line will be carried out in a different paper.

Since the well-posedness of our FBSDEs is based on the Lyapunov operators, the existence of Lypunov operators becomes a crucial question. For decoupled and uniform monotone cases, one can construct a proper Lyapunov operator without much difficulty. We will make some effort to discuss the so-called partial-monotone cases. It turns out that as long as the "nonmonotone" part can be compensated in some way, one can still construct a proper Lyapunov operator. Furthermore, we will look at generators admitting the so-called diagonal Lyapunov operators, which can be used for those generators for which no partial monotonicity conditions are satisfied. The detailed discussion on the existence of Lyapunov operators is one of the major contributions of the present paper.

The rest of this paper is organized as follows. In Section 2, we will briefly present two motivations for considering FBSDE (1.1). In Section 3, the notion of a Lyapunov operator will be introduced. Section 4 is devoted to the well-posedness of FBSDE (1.1) via the method of continuation. The existence of Lyapunov operators 
under various situations will be discussed in Sections 5 and 6 . An illustrative example will be presented in Section 7.

\section{Motivations From StOchastic optimal CONTROL THEORY}

In this section, we briefly present two motivations leading to the study of FBSDEs with mixed initial-terminal conditions.

First, we consider a controlled SDE:

$$
d X(t)=[b(t, X(t))+B(t) u(t)] d t+[\sigma(t, X(t))+D(t) u(t)] d W(t), t \in[0, T],
$$

where $X(\cdot)$ is called the state process and $u(\cdot)$ is called the control process, taking values in $\mathbb{R}^{n}$ and $\mathbb{R}^{m}$, respectively. Consider the following cost functional:

$$
J(u(\cdot))=\mathbb{E}\left\{\int_{0}^{T}\left[Q(t, X(t))+\frac{1}{2}|u(t)|^{2}\right] d t+\frac{1}{2}|X(T)-G X(0)|^{2}\right\},
$$

where $\mathbb{E}$ stands for the expectation with respect to the given probability measure $\mathbb{P}$. The interested optimal control problem is to minimize (2.2) subject to (2.1). We point out that for optimal control of ordinary differential equations, the cost functional containing the term $f(X(0), X(T))$ is pretty standard (see [4], for example). However, in the classical stochastic case, one usually only considers the case that $f(X(0), X(T))=f(X(T))$ (independent of $X(0)$ ) (see [30]). Note that the above cost functional containing the term $|X(T)-G X(0)|^{2}$ means that one would like the terminal state $X(T)$ to be close to $G X(0)$. (This will be the case if, say, $X(\cdot)$ represents the vector of the production level of certain products, and one would like it to grow steadily and healthily. In general, one would like $G$ to be larger than the identity $I$ in some sense.) We suppose that all the involved functions have needed derivatives. Now, if $u(\cdot)$ is an optimal control and $X(\cdot)$ is the corresponding state process, then by a standard variational technique (similar to [30]; see the appendix at the end of this paper), we can derive the following optimality system:

(2.3)

$$
\left\{\begin{aligned}
& d X(t)= {\left[b(t, X(t))-B(t) B(t)^{T} Y(t)-B(t) D(t)^{T} Z(t)\right] d t } \\
&+\left[\sigma(t, X(t))-D(t) B(t)^{T} Y(t)-D(t) D(t)^{T} Z(t)\right] d W(t), \\
& d Y(t)=- {\left[b_{x}(t, X(t))^{T} Y(t)+\sigma_{x}(t, X(t))^{T} Z(t)+Q_{x}(t, X(t))\right] d t+Z(t) d W(t), } \\
& X(0)=\mathbb{E}\left[G^{-1} X(T)-\left(G^{T} G\right)^{-1} Y(0)\right], \\
& Y(T)=X(T)-\mathbb{E} X(T)+\left(G^{T}\right)^{-1} Y(0) .
\end{aligned}\right.
$$

Here, we have assumed that $G^{-1}$ exists. The above is a coupled FBSDE with mixed initial-terminal conditions. If $(X(\cdot), Y(\cdot), Z(\cdot))$ is the unique adapted solution to the above, and optimal control exists, then the control process must be given by

$$
u(t)=-B(t)^{T} Y(t)-C(t)^{T} Z(t), \quad t \in[0, T], \quad \text { a.s. }
$$

Hence, the problem of finding optimal control is reduced to solving the above FBSDE (with mixed initial-terminal conditions). Clearly, (2.3) is a special case of (1.1) with

$$
\gamma(\xi, y)=G^{-1} \mathbb{E} \xi-\left(G^{T} G\right)^{-1} y, h(\xi, y)=\xi-\mathbb{E} \xi+\left(G^{T}\right)^{-1} y, \forall(\xi, y) \in \mathcal{X}^{2} \times \mathbb{R}^{n},
$$


where $\mathcal{X}^{2}$ is the set of all square-integrable $\mathcal{F}_{T}$-measurable $\mathbb{R}^{n}$-valued random variables.

Next, let us look at another interesting motivation. Consider the following controlled SDE:

$$
\left\{\begin{array}{l}
d X(t)=b(t, X(t), u(t)) d t+\sigma(t, X(t), u(t)) d W(t), \quad t \in[0, T] \\
X(0)=X_{0}
\end{array}\right.
$$

with the following payoff functional:

$$
J(u(\cdot))=\mathbb{E}\left\{\int_{0}^{T} f(t, X(t), u(t)) d t+h(X(T))\right\} .
$$

The functional (2.6) represents the preferences of the controller. Between any two controls, the controller prefers the one that has a larger value for the functional $J(\cdot)$. Hence, $f$ and $h$ can be regarded as a representation of the preferences for the controller. Consequently, they could be very subjective. Different groups of people might have quite different preferences. Essentially, one can regard (2.6) as a sort of utility resulting from taking the control $u(\cdot)$. Therefore, (2.6) is a generalization of the von Neumann-Morgenstern's expected utility (27]).

Since the early 1950s, there have appeared the well-known Allais' ([1]) and Ellsberg's (11]) paradoxes, which indicate that linear expected utility theories (by which we mean that in (2.6) the (linear) expectation $\mathbb{E}$ is used) do not well represent people's preferences in many situations. One way to remedy that is to replace the linear expectation by a nonlinear one (22]). More precisely, let $g(t, y, z):[0, T] \times \mathbb{R} \times \mathbb{R} \rightarrow \mathbb{R}$ be measurable, uniformly Lipschitz continuous in $(y, z)$, and $g(t, y, 0)=0$. Let $p>1$. For any $\xi \in L_{\mathcal{F}_{T}}^{p}(\Omega ; \mathbb{R})$, the set of all $\mathcal{F}_{T^{-}}$ measurable random variables which are $L^{p}$-integrable, we let $(Y(\cdot), Z(\cdot))$ be the unique adapted solution of the following BSDE:

$$
\left\{\begin{array}{l}
d Y(t)=g(t, Y(t), Z(t)) d t+Z(t) d W(t), \quad t \in[0, T], \\
Y(T)=\xi
\end{array}\right.
$$

We define the $g$-expectation of $\xi$ by the following:

$$
\mathcal{E}_{g}[\xi]=Y(0) .
$$

It was proved $([22])$ that the operator $\mathcal{E}_{g}: L_{\mathcal{F}_{T}}^{p}(\Omega) \rightarrow \mathbb{R}$ has all the properties of $\mathbb{E}$, except the linearity property. According to [9], [5], 25], [17], [16], and [26], we could refer to $Y(0)$ as the (initial) differential utility of the future payoff $\xi$. We now replace (2.6) by the following:

$$
J(u(\cdot) ; g)=\mathcal{E}_{g}\left\{\int_{0}^{T} f(t, X(t), u(t)) d t+h(X(T))\right\}=Y(0),
$$

where $(Y(\cdot), Z(\cdot))$ is the unique adapted solution to the following BSDE:

$$
\left\{\begin{array}{l}
d Y(t)=g(t, Y(t), Z(t)) d t+Z(t) d W(t), \quad t \in[0, T], \\
Y(T)=\int_{0}^{T} f(t, X(t), u(t)) d t+h(X(T)) .
\end{array}\right.
$$

If we define

$$
X^{0}(t)=\int_{0}^{t} f(s, X(s), u(s)) d s, \quad t \in[0, T]
$$


then the original problem becomes to maximize

$$
J(u(\cdot) ; g)=Y(0) \equiv \mathcal{E}_{g}\left[X^{0}(T)+h(X(T))\right],
$$

subject to the state equation:

$$
\left\{\begin{array}{l}
d X^{0}(t)=f(t, X(t), u(t)) d t \\
d X(t)=b(t, X(t), u(t)) d t+\sigma(t, X(t), u(t)) d W(t) \\
d Y(t)=g(t, Y(t), Z(t)) d t+Z(t) d W(t), \quad t \in[0, T] \\
X(0)=X_{0}, \quad Y(T)=X^{0}(T)+h(X(T))
\end{array}\right.
$$

Thus we end up with an optimal control problem for an FBSDE, with state process $\left(X^{0}(\cdot), X(\cdot), Y(\cdot), Z(\cdot)\right)$ and control process $u(\cdot)$. It is not hard to imagine that the stochastic differential utility process $Y(\cdot)$ could affect the process $X(\cdot)$. When this happens, the forward equation in (2.13) will depend on $Y(\cdot)$, and we end up with a coupled FBSDE. We point out that by using the so-called forward-backward stochastic differential utility ([3]), one will also be led to a coupled FBSDE. Furthermore, the initial differential utility $Y(0)$ of the future payoff might already be taken into account at time $t=0$, which means that $X(0)$ could depend on $Y(0)$. Now, if, in addition, $(X(0), X(T))$ has to satisfy certain constraints (which is already the case for ODE control problems), represented by $\mathbb{E} \varphi(X(0), X(T))=0$, say, then, taking into account the possible dependence of $X(0)$ on $Y(0)$, we might want to have $X(0)=\gamma(X(T), Y(0))$ for some map $\gamma(\cdot)$. Finally, it is quite plausible that the payoff $Y(T)$ at the final time $T$ should depend directly on the initial state $X(0)$. Combining the above, we end up with a controlled FBSDE:

$$
\left\{\begin{array}{l}
d X(t)=b(t, X(t), Y(t), Z(t), u(t)) d t+\sigma(t, X(t), Y(t), Z(t), u(t)) d W(t) \\
d Y(t)=g(t, X(t), Y(t), Z(t), u(t)) d t+Z(t) d W(t) \\
X(0)=\gamma(X(T), Y(0)), \quad Y(T)=h(X(T), Y(0))
\end{array}\right.
$$

with the payoff functional, to be maximized, given by the following:

$$
J(u(\cdot))=\mathbb{E}[f(X(T), Y(0))]
$$

In (2.14) , the state process is any adapted solution $(X(\cdot), Y(\cdot), Z(\cdot))$ and the control process is $u(\cdot)$.

To study the above optimal control problem, we first need to study the wellposedness of FBSDEs with mixed initial-terminal conditions. This gives our second main motivation of the current paper.

\section{Preliminaries}

The purpose of this section is to present some preliminary results, including the introduction of Lyapunov operators. To begin with, let us first introduce some spaces. For any Euclidean space $H$ (with norm $|\cdot|$ ) and $p, r \geq 1$, we define the 
following basic spaces:

$$
\begin{array}{r}
L_{\mathcal{F}_{T}}^{p}(\Omega ; H)=\left\{\xi: \Omega \rightarrow H \mid \xi \text { is } \mathcal{F}_{\left.T^{-} \text {-measurable, } \mathbb{E}|\xi|^{p}<\infty\right\},},\right. \\
L_{\mathbb{F}}^{p}\left(\Omega ; L^{r}(0, T ; H)\right)=\{\varphi:[0, T] \times \Omega \rightarrow H \mid \varphi(\cdot) \text { is } \mathbb{F} \text {-adapted, } \\
\left.\mathbb{E}\left(\int_{0}^{T}|\varphi(t)|^{r} d t\right)^{\frac{p}{r}}<\infty\right\}, \\
L_{\mathbb{F}}^{p}(\Omega ; C([0, T] ; H))=\left\{\varphi(\cdot) \in L_{\mathbb{F}}^{p}(0, T ; H) \mid t \mapsto \varphi(t)\right. \text { is continuous, } \\
\left.\mathbb{E}\left[\sup _{t \in[0, T]}|\varphi(t)|^{p}\right]<\infty\right\} .
\end{array}
$$

We will denote

$$
L_{\mathbb{F}}^{p}(0, T ; H)=L_{\mathbb{F}}^{p}\left(\Omega ; L^{p}(0, T ; H)\right) .
$$

Next, we denote $\mathcal{X}_{n}^{p \triangleq} L_{\mathcal{F}_{T}}^{p}\left(\Omega ; \mathbb{R}^{n}\right) \equiv\left[L_{\mathcal{F}_{T}}^{p}(\Omega ; \mathbb{R})\right]^{n}$, and we define

(3.1) $\mathcal{M}^{p}[0, T] \triangleq L_{\mathbb{F}}^{p}\left(\Omega ; C\left([0, T] ; \mathbb{R}^{n}\right)\right) \times L_{\mathbb{F}}^{p}\left(\Omega ; C\left([0, T] ; \mathbb{R}^{m}\right)\right) \times L_{\mathbb{F}}^{p}\left(\Omega ; L^{2}\left(0, T ; \mathbb{R}^{m}\right)\right)$.

Any process in $\mathcal{M}^{p}[0, T]$ is denoted by $\Theta(\cdot) \equiv(X(\cdot), Y(\cdot), Z(\cdot))$, and we define

$$
\|\Theta(\cdot)\|_{p} \triangleq\left\{\mathbb{E}\left[\sup _{t \in[0, T]}|X(t)|^{p}+\sup _{t \in[0, T]}|Y(t)|^{p}+\left(\int_{0}^{T}|Z(t)|^{2} d t\right)^{\frac{p}{2}}\right]\right\}^{\frac{1}{p}} .
$$

Clearly, (3.2) is a norm under which $\mathcal{M}^{p}[0, T]$ is a Banach space. By an $L^{p_{-}}$ adapted solution (or simply adapted solution) to FBSDE (1.1) we mean a process $\Theta(\cdot) \equiv(X(\cdot), Y(\cdot), Z(\cdot)) \in \mathcal{M}^{p}[0, T]$ that satisfies (1.1) in the usual Itô sense (see [20]). For convenience, hereafter, any generic point in $\mathbb{M} \equiv \mathbb{R}^{n} \times \mathbb{R}^{m} \times \mathbb{R}^{m}$ will be denoted by $\theta \equiv(x, y, z)$, and the set of all $(k \times k)$ symmetric matrices will be denoted by $\mathcal{S}^{k}$. For any $A \in \mathcal{S}^{k}$, by $A \geq 0$ we mean that $A$ is positive semi-definite, and $A \leq 0$ if $-A \geq 0$. We let $\mathcal{S}_{+}^{k}$ be the set of all $(k \times k)$ positive semi-definite matrices. If $\Phi$ is a function or process valued in $\mathcal{S}^{k}$, by $\Phi \gg 0$, we mean that there exists a $\rho>0$ such that $\Phi \geq \rho I$ for all (or almost all) the arguments of $\Phi$ in its domain, and $\Phi \ll 0$ means that $-\Phi \gg 0$. The meaning $\Phi \gg \Psi$ is $\Phi-\Psi \gg 0$, for any processes $\Phi$ and $\Psi$ valued in the same space $\mathcal{S}^{k}$. With such an order, one can naturally define convexity/concavity for functions $\Phi: \mathbb{R}^{n} \rightarrow \mathcal{S}^{k}$. Moreover, if $\Phi: \mathcal{Z} \rightarrow \mathcal{S}_{+}^{k}$ is a bounded map with $\mathcal{Z}$ being some nonempty set, we let $\{\Phi\}_{\infty}$ be the smallest $\Psi \in \mathcal{S}_{+}^{k}$ such that $\Psi \geq \Phi(z)$ for all $z \in \mathcal{Z}$.

For $p \geq 1$, let $\mathcal{G}_{0}^{p}$ be the set of all 5 -tuples $\left(b_{0}, \sigma_{0}, g_{0}, \gamma_{0}, h_{0}\right)$ satisfying the following:

$$
\left\{\begin{array}{l}
b_{0}(\cdot) \in L_{\mathbb{F}}^{p}\left(\Omega ; L^{1}\left(0, T ; \mathbb{R}^{n}\right)\right), \quad \sigma_{0}(\cdot) \in L_{\mathbb{F}}^{p}\left(\Omega ; L^{2}\left(0, T ; \mathbb{R}^{n}\right)\right), \\
g_{0}(\cdot) \in L_{\mathbb{F}}^{p}\left(\Omega ; L^{1}\left(0, T ; \mathbb{R}^{m}\right)\right), \quad \gamma_{0} \in \mathbb{R}^{n}, \quad h_{0} \in \mathcal{X}_{m}^{p}
\end{array}\right.
$$

Next, we introduce the following standing assumption. Let $p \geq 1$.

$(\mathbf{H 1})_{p}$ The following maps,

$$
\left\{\begin{array}{l}
b, \sigma:[0, T] \times \mathbb{M} \times \Omega \rightarrow \mathbb{R}^{n}, \quad g:[0, T] \times \mathbb{M} \times \Omega \rightarrow \mathbb{R}^{m}, \\
\gamma: \mathcal{X}_{n}^{p} \times \mathbb{R}^{m} \rightarrow \mathbb{R}^{n}, \quad h: \mathcal{X}_{n}^{p} \times \mathbb{R}^{m} \times \Omega \rightarrow \mathcal{X}_{m}^{p},
\end{array}\right.
$$

are all measurable. For each $\theta \in \mathbb{M}$, the process $t \mapsto(b(t, \theta), \sigma(t, \theta), g(t, \theta))$ is $\mathbb{F}$-progressively measurable, and $\omega \mapsto h(x, y, \omega)$ is $\mathcal{F}_{T}$-measurable, such that

$$
(b(\cdot, 0), \sigma(\cdot, 0), g(\cdot, 0), \gamma(0), h(0)) \in \mathcal{G}_{0}^{p} .
$$


For almost all $(t, \omega) \in[0, T] \times \Omega, \theta \mapsto(b(t, \theta, \omega), \sigma(t, \theta, \omega), g(t, \theta, \omega)),(\xi, y) \mapsto \gamma(\xi, y)$, and $(\xi, y) \mapsto h(\xi, y)$ are all (Frechét) differentiable, with all involved (Frechét) derivatives uniformly bounded.

Note that in (3.5),

$$
\begin{aligned}
& b(\cdot, 0)=\left.b(\cdot, \theta)\right|_{\theta=0}, \quad \sigma(\cdot, 0)=\left.\sigma(\cdot, \theta)\right|_{\theta=0}, \quad g(\cdot, 0)=\left.g(\cdot, \theta)\right|_{\theta=0}, \\
& \gamma(0)=\left.\gamma(\xi, y)\right|_{\xi=0, y=0}, \quad h(0)=\left.h(\xi, y)\right|_{\xi=0, y=0} .
\end{aligned}
$$

Thus, $\gamma(0)$ is deterministic and $h(0)$ is $\mathcal{F}_{T}$-measurable. For convenience, we denote the set of all generators $(b, \sigma, g, \gamma, h)$ satisfying $(\mathrm{H} 1)_{p}$ by $\mathcal{G}^{p}$.

For any $(b, \sigma, g, \gamma, h) \in \mathcal{G}^{p},\left(b_{0}, \sigma_{0}, g_{0}, \gamma_{0}, h_{0}\right) \in \mathcal{G}_{0}^{p}$, and $\rho \in[0,1]$, consider the following FBSDE (compare with (1.1)):

$$
\left\{\begin{array}{l}
d X(t)=\left[\rho b(t, \Theta(t))+b_{0}(t)\right] d t+\left[\rho \sigma(t, \Theta(t))+\sigma_{0}(t)\right] d W(t), \\
d Y(t)=\left[\rho g(t, \Theta(t))+g_{0}(t)\right] d t+Z(t) d W(t), \\
X(0)=\rho \gamma(X(T), Y(0))+\gamma_{0}, \quad Y(T)=\rho h(X(T), Y(0))+h_{0} .
\end{array}\right.
$$

It is easy to see that when $\rho=0$, (3.7) is a trivial FBSDE which admits a unique adapted solution, and when $\rho=1$, (3.7) is essentially the same as (although it looks a little more general than) (1.1). Under certain conditions, we can show that when (3.7) is (uniquely) solvable for some $\rho \in[0,1)$, it is (uniquely) solvable for (3.7) with $\rho$ replaced by $(\rho+\varepsilon) \wedge 1$, with $\varepsilon>0$ being an absolute constant. Then, by induction, we obtain the (unique) solvability of FBSDE (1.1) over $[0, T]$. Such an argument is called a method of continuation (see [28]). In doing so, the key is to establish an a priori estimate for the adapted solutions to (3.7) uniform in $\rho \in[0,1]$. To this end, we need to make a little preparation.

Suppose

$$
\left\{\begin{array}{l}
(b, \sigma, g, \gamma, h),(\bar{b}, \bar{\sigma}, \bar{g}, \bar{\gamma}, \bar{h}) \in \mathcal{G}^{2}, \\
\left(b_{0}(\cdot), \sigma_{0}(\cdot), g_{0}(\cdot), \gamma_{0}, h_{0}\right),\left(\bar{b}_{0}(\cdot), \bar{\sigma}_{0}(\cdot), \bar{g}_{0}(\cdot), \bar{\gamma}_{0}, \bar{h}_{0}\right) \in \mathcal{G}_{0}^{2}
\end{array}\right.
$$

Let $\Theta_{\rho}(\cdot) \equiv\left(X_{\rho}(\cdot), Y_{\rho}(\cdot), Z_{\rho}(\cdot)\right), \bar{\Theta}_{\rho}(\cdot) \equiv\left(\bar{X}_{\rho}(\cdot), \bar{Y}_{\rho}(\cdot), \bar{Z}_{\rho}(\cdot)\right) \in \mathcal{M}^{2}[0, T]$ be adapted solutions of FBSDE (3.7) corresponding to the generators $(b, \sigma, g, \gamma, h)$ and $(\bar{b}, \bar{\sigma}$, $\bar{g}, \bar{\gamma}, \bar{h})$, and the nonhomogeneous processes $\left(b_{0}(\cdot), \sigma_{0}(\cdot), g_{0}(\cdot), \gamma_{0}, h_{0}\right)$ and $\left(\bar{b}_{0}(\cdot)\right.$, $\left.\bar{\sigma}_{0}(\cdot), \bar{g}_{0}(\cdot), \bar{\gamma}_{0}, \bar{h}_{0}\right)$, respectively. Denote

$$
\left\{\begin{array}{l}
\widehat{\Theta}(t) \equiv \widehat{\Theta}_{\rho}(\cdot) \triangleq \bar{\Theta}_{\rho}(t)-\Theta_{\rho}(t) \\
\widehat{X}(\cdot)=\bar{X}_{\rho}(\cdot)-X_{\rho}(\cdot), \quad \widehat{Y}(\cdot)=\bar{Y}_{\rho}(\cdot)-Y_{\rho}(\cdot), \quad \widehat{Z}(\cdot)=\bar{Z}_{\rho}(\cdot)-Z_{\rho}(\cdot) .
\end{array}\right.
$$

Set

$$
\left\{\begin{array}{l}
\widehat{b}(t)=\bar{b}\left(t, \bar{\Theta}_{\rho}(t)\right)-b\left(t, \Theta_{\rho}(t)\right), \quad \widehat{\sigma}(t)=\bar{\sigma}\left(t, \bar{\Theta}_{\rho}(t)\right)-\sigma\left(t, \Theta_{\rho}(t)\right), \\
\widehat{g}(t)=\bar{g}\left(t, \bar{\Theta}_{\rho}(t)\right)-g\left(t, \Theta_{\rho}(t)\right), \quad \widehat{\gamma}=\bar{\gamma}\left(\bar{X}_{\rho}(T), \bar{Y}_{\rho}(0)\right)-\gamma\left(X_{\rho}(T), Y_{\rho}(0)\right), \\
\widehat{h}=\bar{h}\left(\bar{X}_{\rho}(T), \bar{Y}_{\rho}(0)\right)-h\left(X_{\rho}(T), Y_{\rho}(0)\right),
\end{array}\right.
$$


and

$$
\left\{\begin{array}{l}
\delta b(t)=\bar{b}\left(t, \bar{\Theta}_{\rho}(t)\right)-b\left(t, \bar{\Theta}_{\rho}(t)\right), \quad \delta b_{0}(t)=\bar{b}_{0}(t)-b_{0}(t), \\
\delta \sigma(t)=\bar{\sigma}\left(t, \bar{\Theta}_{\rho}(t)\right)-\sigma\left(t, \bar{\Theta}_{\rho}(t)\right), \quad \delta \sigma_{0}(t)=\bar{\sigma}_{0}(t)-\sigma_{0}(t), \\
\delta g(t)=\bar{g}\left(t, \bar{\Theta}_{\rho}(t)\right)-g\left(t, \bar{\Theta}_{\rho}(t)\right), \quad \delta g_{0}(t)=\bar{g}_{0}(t)-g_{0}(t), \\
\delta \gamma=\bar{\gamma}\left(\bar{X}_{\rho}(T), \bar{Y}_{\rho}(0)\right)-\gamma\left(\bar{X}_{\rho}(T), \bar{Y}_{\rho}(0)\right), \quad \delta \gamma_{0}=\bar{\gamma}_{0}-\gamma_{0}, \\
\delta h=\bar{h}\left(\bar{X}_{\rho}(T), \bar{Y}_{\rho}(0)\right)-h\left(\bar{X}_{\rho}(T), \bar{Y}_{\rho}(0)\right), \quad \delta h_{0}=\bar{h}_{0}-h_{0} .
\end{array}\right.
$$

Furthermore, for $f(\cdot)=\gamma(\cdot), h(\cdot)$, let

$$
\begin{cases}\widetilde{f}_{\xi}=\int_{0}^{1} f_{\xi}^{\alpha} d \alpha, & f_{\xi}^{\alpha}=f_{\xi}\left(X_{\rho}(T)+\alpha \widehat{X}(T), Y_{\rho}(0)+\alpha \widehat{Y}(0)\right), \\ \widetilde{f}_{y}=\int_{0}^{1} f_{y}^{\alpha} d \alpha, & f_{y}^{\alpha}=f\left(X_{\rho}(T)+\alpha \widehat{X}(T), Y_{\rho}(0)+\alpha \widehat{Y}(0)\right),\end{cases}
$$

and for $f(\cdot)=b(\cdot), \sigma(\cdot), g(\cdot)$, let

$$
\begin{cases}\widetilde{f}_{x}(t)=\int_{0}^{1} f_{x}^{\alpha}(t) d \alpha, & f_{x}^{\alpha}(t)=f_{x}\left(t, \Theta_{\rho}(t)+\alpha \widehat{\Theta}(t)\right), \\ \widetilde{f}_{y}(t)=\int_{0}^{1} f_{y}^{\alpha}(t) d \alpha, & f_{y}^{\alpha}(t)=f_{y}\left(t, \Theta_{\rho}(t)+\alpha \widehat{\Theta}(t)\right), \\ \widetilde{f}_{z}(t)=\int_{0}^{1} f_{z}^{\alpha}(t) d \alpha, & f_{z}^{\alpha}(t)=f_{z}\left(t, \Theta_{\rho}(t)+\alpha \widehat{\Theta}(t)\right) .\end{cases}
$$

In what follows, for any bounded random field $f(\cdot)$, let $\|f\|_{\infty}$ be its essential bounded; for any $\Phi \in \mathcal{S}^{k}$, let $\Lambda(\Phi) \subset \mathbb{R}$ be the set of all its eigenvalues. We will let $K$ be a generic constant which can be different from line to line. With the above notation, we have the following result.

Proposition 3.1. (i) For $\rho \in[0,1]$, let

$$
\begin{array}{r}
\left.L_{b \sigma}^{\rho}(t) \triangleq \underset{\theta \in \mathbb{M}, \omega \in \Omega}{\triangleq} \operatorname{ess} \max \Lambda\left(b_{x}(t, \theta, \omega)+b_{x}(t, \theta, \omega)^{T}+\rho \sigma_{x}(t, \theta, \omega)^{T} \sigma_{x}(t, \theta, \omega)\right)\right], \\
t \in[0, T],
\end{array}
$$

satisfy

$$
\left[\sup _{\rho \in[0,1]} \rho^{2} e^{\int_{0}^{T} L_{b \sigma}^{\rho}(t) d t}\right]\left\|\gamma_{\xi}\right\|_{\infty}^{2}<1
$$

Then

$$
\begin{gathered}
\mathbb{E}\left[\sup _{t \in[0, T]}|\widehat{X}(t)|^{2}\right] \leq K \mathbb{E}\left[\left|\widetilde{\gamma}_{y} \widehat{Y}(0)\right|^{2}+|\delta \gamma|^{2}+\left|\delta \gamma_{0}\right|^{2}\right. \\
+\int_{0}^{T}\left(\left|\widetilde{b}_{y}(t) \widehat{Y}(t)+\widetilde{b}_{z}(t) \widehat{Z}(t)\right|^{2}+\left|\widetilde{\sigma}_{y}(t) \widehat{Y}(t)+\widetilde{\sigma}_{z}(t) \widehat{Z}(t)\right|^{2}\right. \\
\left.\left.+|\delta b(t)|^{2}+\left|\delta b_{0}(t)\right|^{2}+|\delta \sigma(t)|^{2}+\left|\delta \sigma_{0}(t)\right|^{2}\right) d t\right] .
\end{gathered}
$$

(ii) For $\rho \in[0,1]$, let

$$
\begin{array}{r}
L_{g}^{\rho}(t) \triangleq \rho_{\theta \in \mathbb{M}, \omega \in \Omega} \operatorname{ess} \sup \left[\max \Lambda\left(-g_{y}(t, \theta, \omega)-g_{y}(t, \theta, \omega)^{T}+\rho g_{z}(t, \theta, \omega) g_{z}(t, \theta, \omega)^{T}\right)\right], \\
t \in[0, T],
\end{array}
$$


satisfy

$$
\left[\sup _{\rho \in[0,1]} \rho^{2} e^{\int_{0}^{T} L_{g}^{\rho}(t) d t}\right]\left\|h_{y}\right\|_{\infty}^{2}<1, \quad \rho \in[0,1]
$$

Then

$$
\begin{aligned}
\mathbb{E}\left[\sup _{t \in[0, T]}|\widehat{Y}(t)|^{2}+\int_{0}^{T}|\widehat{Z}(t)|^{2} d t\right] \leq K \mathbb{E}\left[\left|\widetilde{h}_{\xi} \widehat{X}(T)\right|^{2}+|\delta h|^{2}+\left|\delta h_{0}\right|^{2}\right. \\
\left.+\int_{0}^{T}\left(\left|\widetilde{g}_{x}(t) \widehat{X}(t)\right|^{2}+|\delta g(t)|^{2}+\left|\delta g_{0}(t)\right|^{2}\right) d t\right] .
\end{aligned}
$$

(iii) Let both (3.15) and (3.18) hold. Then

$$
\begin{aligned}
& \mathbb{E}\left[\sup _{t \in[0, T]}|\widehat{X}(t)|^{2}+\sup _{t \in[0, T]}|\widehat{Y}(t)|^{2}+\int_{0}^{T}|\widehat{Z}(t)|^{2} d t\right] \\
& \leq K \mathbb{E}\left[\left|\widetilde{\gamma}_{y} \widehat{Y}(0)\right|^{2}+|\delta \gamma|^{2}+\left|\delta \gamma_{0}\right|^{2}+|\delta h|^{2}+\left|\delta h_{0}\right|^{2}\right. \\
& \quad+\int_{0}^{T}\left(\left|\widetilde{b}_{y}(t) \widehat{Y}(t)+\widetilde{b}_{z}(t) \widehat{Z}(t)\right|^{2}+\left|\widetilde{\sigma}_{y}(t) \widehat{Y}(t)+\widetilde{\sigma}_{z}(t) \widehat{Z}(t)\right|^{2}\right. \\
& \left.\left.\quad+|\delta b(t)|^{2}+\left|\delta b_{0}(t)\right|^{2}+|\delta \sigma(t)|^{2}+\left|\delta \sigma_{0}(t)\right|^{2}+|\delta g(t)|^{2}+\left|\delta g_{0}(t)\right|^{2}\right) d t\right],
\end{aligned}
$$

and

$$
\begin{aligned}
& \mathbb{E}\left[\sup _{t \in[0, T]}|\widehat{X}(t)|^{2}+\sup _{t \in[0, T]}|\widehat{Y}(t)|^{2}+\int_{0}^{T}|\widehat{Z}(t)|^{2} d t\right] \\
& \leq K \mathbb{E}\left[\left|\widetilde{h}_{\xi} \widehat{X}(T)\right|^{2}+|\delta \gamma|^{2}+\left|\delta \gamma_{0}\right|^{2}+|\delta h|^{2}+\left|\delta h_{0}\right|^{2}+\int_{0}^{T}\left(\left|\widetilde{g}_{x}(t) \widehat{X}(t)\right|^{2}\right.\right. \\
& \left.\left.\quad+|\delta b(t)|^{2}+\left|\delta b_{0}(t)\right|^{2}+|\delta \sigma(t)|^{2}+\left|\delta \sigma_{0}(t)\right|^{2}+|\delta g(t)|^{2}+\left|\delta g_{0}(t)\right|^{2}\right) d t\right] .
\end{aligned}
$$

Proof. (i) Applying Itô's formula to $|\widehat{X}(\cdot)|^{2}$, we have

$$
\begin{aligned}
& \mathbb{E}|\widehat{X}(t)|^{2}=|\widehat{X}(0)|^{2}+\mathbb{E} \int_{0}^{t}\left(2\left\langle\widehat{X}(s), \rho \widehat{b}(s)+\delta b_{0}(s)\right\rangle+\left|\rho \widehat{\sigma}(s)+\delta \sigma_{0}(s)\right|^{2}\right) d s \\
& \leq|\widehat{X}(0)|^{2}+\mathbb{E} \int_{0}^{t}\left(2\left\langle\widehat{X}(s), \rho \widetilde{b}_{x}(s) \widehat{X}(s)+\rho \widetilde{b}_{y}(s) \widehat{Y}(s)+\rho \widetilde{b}_{z}(s) \widehat{Z}(s)+\rho \delta b(s)+\delta b_{0}(s)\right\rangle\right. \\
& \left.\quad+\left|\rho \widetilde{\sigma}_{x}(s) \widehat{X}(s)+\rho \widetilde{\sigma}_{y}(s) \widehat{Y}(s)+\rho \widetilde{\sigma}_{z}(s) \widehat{Z}(s)+\rho \delta \sigma(s)+\delta \sigma_{0}(s)\right|^{2}\right) d s \\
& \leq|\widehat{X}(0)|^{2}+\mathbb{E} \int_{0}^{t}\left\{\left[L_{b \sigma}^{\rho}(s)+\varepsilon\right]|\widehat{X}(s)|^{2}+K_{\varepsilon}\left[\widetilde{b}_{y}(s) \widehat{Y}(s)+\left.\widetilde{b}_{z}(s) \widehat{Z}(s)\right|^{2}\right.\right. \\
& \left.\left.\quad+\left|\widetilde{\sigma}_{y}(s) \widehat{Y}(s)+\widetilde{\sigma}_{z}(s) \widehat{Z}(s)\right|^{2}+|\delta b(s)|^{2}+\left|\delta b_{0}(s)\right|^{2}+|\delta \sigma(s)|^{2}+\left|\delta \sigma_{0}(s)\right|^{2}\right]\right\} d s
\end{aligned}
$$

where $\varepsilon>0$ is small and $K_{\varepsilon}>0$ is a constant depending on $\varepsilon>0$. By Gronwall's inequality, one obtains that for any $t \in[0, T]$,

$$
\begin{aligned}
\mathbb{E}|\widehat{X}(t)|^{2} \leq & e^{\int_{0}^{t}\left[L_{b \sigma}^{\rho}(s)+\varepsilon\right] d s}|\widehat{X}(0)|^{2}+K_{\varepsilon} \int_{0}^{t}\left(\left|\widetilde{b}_{y}(s) \widehat{Y}(s)+\widetilde{b}_{z}(s) \widehat{Z}(s)\right|^{2}\right. \\
& \left.+\left|\widetilde{\sigma}_{y}(s) \widehat{Y}(s)+\widetilde{\sigma}_{z}(s) \widehat{Z}(s)\right|^{2}+|\delta b(s)|^{2}+\left|\delta b_{0}(s)\right|^{2}+|\delta \sigma(s)|^{2}+\left|\delta \sigma_{0}(s)\right|^{2}\right) d s .
\end{aligned}
$$


In particular, at $t=T$, noting the initial condition,

$$
\begin{aligned}
& \mathbb{E}|\widehat{X}(T)|^{2} \leq e^{\int_{0}^{T}\left[L_{b \sigma}^{\rho}(s)+\varepsilon\right] d s}\left|\rho\left[\widetilde{\gamma}_{\xi} \widehat{X}(T)+\widetilde{\gamma}_{y} \widehat{Y}(0)+\delta \gamma\right]+\delta \gamma_{0}\right|^{2} \\
& +K \int_{0}^{T}\left(\left|\widetilde{b}_{y}(s) \widehat{Y}(s)+\widetilde{b}_{z}(s) \widehat{Z}(s)\right|^{2}+\left|\widetilde{\sigma}_{y}(s) \widehat{Y}(s)+\widetilde{\sigma}_{z}(s) \widehat{Z}(s)\right|^{2}\right. \\
& \left.+|\delta b(s)|^{2}+\left|\delta b_{0}(s)\right|^{2}+|\delta \sigma(s)|^{2}+\left|\delta \sigma_{0}(s)\right|^{2}\right) d s \\
& \leq(1+\varepsilon) \rho^{2} e^{\int_{0}^{T}\left[L_{b \sigma}^{\rho}(s)+\varepsilon\right] d s}\left\|\gamma_{\xi}\right\|_{\infty}^{2} \mathbb{E}|\widehat{X}(T)|^{2}+K \mathbb{E}\left[\left|\widetilde{\gamma}_{y} \widehat{Y}(0)\right|^{2}+|\delta \gamma|^{2}+\left|\delta \gamma_{0}\right|^{2}\right. \\
& +\int_{0}^{T}\left(\widetilde{b}_{y}(s) \widehat{Y}(s)+\left.\widetilde{b}_{z}(s) \widehat{Z}(s)\right|^{2}+\left|\widetilde{\sigma}_{y}(s) \widehat{Y}(s)+\widetilde{\sigma}_{z}(s) \widehat{Z}(s)\right|^{2}\right. \\
& \left.\left.+|\delta b(s)|^{2}+\left|\delta b_{0}(s)\right|^{2}+|\delta \sigma(s)|^{2}+\left|\delta \sigma_{0}(s)\right|^{2}\right) d s\right] .
\end{aligned}
$$

Therefore, by (3.15), we have (choosing $\varepsilon>0$ small enough)

$$
\begin{aligned}
& \mathbb{E}|\widehat{X}(T)|^{2} \leq K \mathbb{E}\left[\left|\widetilde{\gamma}_{y} \widehat{Y}(0)\right|^{2}+|\delta \gamma|^{2}+\left|\delta \gamma_{0}\right|^{2}\right. \\
& +\int_{0}^{T}\left(\widetilde{b}_{y}(s) \widehat{Y}(s)+\left.\widetilde{b}_{z}(s) \widehat{Z}(s)\right|^{2}+\left|\widetilde{\sigma}_{y}(s) \widehat{Y}(s)+\widetilde{\sigma}_{z}(s) \widehat{Z}(s)\right|^{2}\right. \\
& \left.\left.+|\delta b(s)|^{2}+\left|\delta b_{0}(s)\right|^{2}+|\delta \sigma(s)|^{2}+\left|\delta \sigma_{0}(s)\right|^{2}\right) d s\right] .
\end{aligned}
$$

Consequently,

$$
\begin{aligned}
& |\widehat{X}(0)|^{2}=\left|\rho \widetilde{\gamma}_{\xi} \widehat{X}(T)+\rho \widetilde{\gamma}_{y} \widehat{Y}(0)+\rho \delta \gamma+\delta \gamma_{0}\right|^{2} \\
& \leq K \mathbb{E}\left[\left|\widetilde{\gamma}_{y} \widehat{Y}(0)\right|^{2}+|\delta \gamma|^{2}+\left|\delta \gamma_{0}\right|^{2}\right. \\
& +\int_{0}^{T}\left(\widetilde{b}_{y}(s) \widehat{Y}(s)+\left.\widetilde{b}_{z}(s) \widehat{Z}(s)\right|^{2}+\left|\widetilde{\sigma}_{y}(s) \widehat{Y}(s)+\widetilde{\sigma}_{z}(s) \widehat{Z}(s)\right|^{2}\right. \\
& \left.\left.+|\delta b(s)|^{2}+\left|\delta b_{0}(s)\right|^{2}+|\delta \sigma(s)|^{2}+\left|\delta \sigma_{0}(s)\right|^{2}\right) d s\right] .
\end{aligned}
$$

Then (3.16) follows from a standard estimate for SDEs.

(ii) Applying Itô's formula to $|\widehat{Y}(\cdot)|^{2}$, we have

$$
\begin{aligned}
& \mathbb{E}|\widehat{Y}(T)|^{2}-\mathbb{E}|\widehat{Y}(t)|^{2}=\mathbb{E} \int_{t}^{T}\left(2\left\langle\widehat{Y}(s), \rho \widehat{g}(s)+\delta g_{0}(s)\right\rangle+|\widehat{Z}(s)|^{2}\right) d s \\
& =\mathbb{E} \int_{t}^{T}\left(2\left\langle\widehat{Y}(s), \rho \widetilde{g}_{x}(s) \widehat{X}(s)+\rho \widetilde{g}_{y}(s) \widehat{Y}(s)+\rho \widetilde{g}_{z}(s) \widehat{Z}(s)+\rho \delta g(s)+\delta g_{0}(s)\right\rangle+|\widehat{Z}(s)|^{2}\right) d s \\
& =\mathbb{E} \int_{t}^{T}\left(\left|\widehat{Z}(s)+\rho \widetilde{g}_{z}(s)^{T} \widehat{Y}(s)\right|^{2}+\left\langle\left[\rho \widetilde{g}_{y}(s)+\rho \widetilde{g}_{y}(s)^{T}-\rho^{2} \widetilde{g}_{z}(s) \widetilde{g}_{z}(s)^{T}\right] \widehat{Y}(s), \widehat{Y}(s)\right\rangle\right. \\
& \left.\quad+2\left\langle\widehat{Y}(s), \rho \widetilde{g}_{x}(s) \widehat{X}(s)+\rho \delta g(s)+\delta g_{0}(s)\right\rangle\right) d s \\
& \geq \mathbb{E} \int_{t}^{T}\left\{\left[-L_{g}^{\rho}(s)-\varepsilon\right]|\widehat{Y}(s)|^{2}-K_{\varepsilon}\left[\left|\widetilde{g}_{x}(s) \widehat{X}(s)\right|^{2}+|\delta g(s)|^{2}+\left|\delta g_{0}(s)\right|^{2}\right]\right\} d s,
\end{aligned}
$$

with $\varepsilon>0$ small. By Gronwall's inequality, for any $t \in[0, T]$,

$$
\mathbb{E}|\widehat{Y}(t)|^{2} \leq \mathbb{E}\left[e^{\int_{t}^{T}\left[L_{g}^{\rho}(s)+\varepsilon\right] d s}|\widehat{Y}(T)|^{2}+K \int_{t}^{T}\left(\left|\widetilde{g}_{x}(s) \widehat{X}(s)\right|^{2}+|\delta g(s)|^{2}+\left|\delta g_{0}(s)\right|^{2}\right) d s\right] .
$$


In particular, by taking $t=0$, one has (noting the terminal condition)

$$
\begin{gathered}
|\widehat{Y}(0)|^{2} \leq \mathbb{E}\left[e^{\int_{0}^{T}\left[L_{g}^{\rho}(s)+\varepsilon\right] d s}\left|\rho^{2}\left[\widetilde{h}_{\xi} \widehat{X}(T)+\widetilde{h}_{y} \widehat{Y}(0)+\delta h\right]+\delta h_{0}\right|^{2}\right. \\
\left.+K \int_{0}^{T}\left(\left|\widetilde{g}_{x}(s) \widehat{X}(s)\right|^{2}+|\delta g(s)|^{2}+\left|\delta g_{0}(s)\right|^{2}\right) d s\right] \\
\leq(1+\varepsilon) \rho^{2} e_{0}^{\int_{0}^{T}\left[L_{g}^{\rho}(s)+\varepsilon\right] d s}\left\|h_{y}\right\|_{\infty}^{2}|\widehat{Y}(0)|^{2}+K \mathbb{E}\left[\left|\widetilde{h}_{\xi} \widehat{X}(T)\right|^{2}+|\delta h|^{2}+\left|\delta h_{0}\right|^{2}\right. \\
\left.+K \int_{0}^{T}\left(\left|\widetilde{g}_{x}(s) \widehat{X}(s)\right|^{2}+|\delta g(s)|^{2}+\left|\delta g_{0}(s)\right|^{2}\right) d s\right] .
\end{gathered}
$$

Consequently, under condition (3.18), we have

$$
|\widehat{Y}(0)|^{2} \leq K \mathbb{E}\left[\left|\widetilde{h}_{\xi} \widehat{X}(T)\right|^{2}+|\delta h|^{2}+\left|\delta h_{0}\right|^{2}+\int_{0}^{T}\left(\left|\widetilde{g}_{x}(s) \widehat{X}(s)\right|^{2}+|\delta g(s)|^{2}+\left|\delta g_{0}(s)\right|^{2}\right) d s\right],
$$

which yields

$$
\begin{aligned}
& \mathbb{E}|\widehat{Y}(T)|^{2}=\mathbb{E}\left|\rho \widetilde{h}_{\xi} \widehat{X}(T)+\rho \widetilde{h}_{y} \widehat{Y}(0)+\rho \delta h+\delta h_{0}\right|^{2} \\
& \leq K \mathbb{E}\left[\left.\widetilde{h}_{\xi} \widehat{X}(T)\right|^{2}+|\delta h|^{2}+\left|\delta h_{0}\right|^{2}+\int_{0}^{T}\left(\left|\widetilde{g}_{x}(s) \widehat{X}(s)\right|^{2}+|\delta g(s)|^{2}+\left|\delta g_{0}(s)\right|^{2}\right) d s\right] .
\end{aligned}
$$

Then by a standard estimate for BSDEs, we obtain (3.19).

(iii) Combining (i) and (ii), together with some standard estimates for SDEs and BSDEs, we can obtain (3.20) and (3.21).

If we denote

$$
\left\{\begin{array}{l}
L_{b \sigma}(t) \triangleq \operatorname{esssup}_{\theta \in \mathbb{M}, \omega \in \Omega}\left[\max \Lambda\left(b_{x}(t, \theta, \omega)+b_{x}(t, \theta, \omega)^{T}+\sigma_{x}(t, \theta, \omega)^{T} \sigma_{x}(t, \theta, \omega)\right)\right], \\
L_{g}(t) \triangleq \operatorname{ess~sup}_{\theta \in \mathbb{M}, \omega \in \Omega}\left[\max \Lambda\left(-g_{y}(t, \theta, \omega)-g_{y}(t, \theta, \omega)^{T}+g_{z}(t, \theta, \omega) g_{z}(t, \theta, \omega)^{T}\right)\right],
\end{array}\right.
$$

then by the definitions of $L_{b \sigma}^{\rho}(t)$ and $L_{g}^{\rho}(t)$ (see (3.14) and (3.17)), we have

$$
L_{b \sigma}(t)=L_{b \sigma}^{1}(t), \quad L_{g}(t)=L_{g}^{1}(t),
$$

and

$$
\int_{0}^{T} L_{b \sigma}^{\rho}(t) d t \leq \rho \int_{0}^{T} L_{b \sigma}(t) d t \equiv \rho \widetilde{L}_{b \sigma}, \quad \int_{0}^{T} L_{g}^{\rho}(t) d t \leq \rho \int_{0}^{T} L_{g}(t) d t \equiv \rho \widetilde{L}_{g} .
$$

Note that $L_{b \sigma}(t)$ and $L_{g}(t)$ are allowed to take negative values, so are numbers $\widetilde{L}_{b \sigma}$ and $\widetilde{L}_{g}$. Now, let us consider the function $f(\rho)=\rho^{2} e^{L \rho}$ for $L \in \mathbb{R}$ ( $L$ could be either positive or negative). Then a direct computation shows that

$$
\max _{\rho \in[0,1]} f(\rho)=\left\{\begin{array}{l}
f(1)=e^{L}, \quad L \geq-2, \\
f\left(\frac{2}{|L|}\right)=\frac{4}{L^{2}} e^{-2}, \quad L<-2 .
\end{array}\right.
$$

This means that

$$
\rho^{2} e^{\rho L} \leq \frac{4}{[(-2) \wedge L]^{2}} e^{(-2) \vee L}, \quad \forall \rho \in[0,1] .
$$

Hence, conditions (3.15) and (3.18) are implied by the following, respectively:

$$
\frac{4}{\left[(-2) \wedge \widetilde{L}_{b \sigma}\right]^{2}} e^{(-2) \vee \widetilde{L}_{b \sigma}}\left\|\gamma_{\xi}\right\|_{\infty}^{2}<1, \quad \frac{4}{\left[(-2) \wedge \widetilde{L}_{g}\right]^{2}} e^{(-2) \vee \widetilde{L}_{g}}\left\|h_{y}\right\|_{\infty}^{2}<1
$$


Although more restrictive, the above two conditions are much easier to check. On the other hand, condition (3.15) trivially holds if $\left\|\gamma_{\xi}\right\|_{\infty}=0$; likewise, (3.18) holds if $\left\|h_{y}\right\|_{\infty}=0$. Hence, for the classical case, i.e., $\left\|\gamma_{\xi}\right\|_{\infty}=\left\|\gamma_{y}\right\|_{\infty}=\left\|h_{y}\right\|=0$, both (3.15) and (3.18) automatically hold.

From (i)-(ii) of the above proposition, we see that due to the coupling of FBSDEs, in general, one can only obtain an estimate of $\widehat{X}(\cdot)$ in terms of $(\widehat{Y}(\cdot), \widehat{Z}(\cdot))$, and an estimate of $(\widehat{Y}(\cdot), \widehat{Z}(\cdot))$ in terms of $\widehat{X}(\cdot)$. From (iii) of the above proposition, we further see that in order to obtain an estimate on $\widehat{\Theta}(\cdot)$, we need to either have an estimate for

$$
\mathbb{E}\left[\left|\widetilde{\gamma}_{y} \widehat{Y}(0)\right|^{2}+\int_{0}^{T}\left(\left|\widetilde{b}_{y}(t) \widehat{Y}(t)+\widetilde{b}_{z}(t) \widehat{Z}(t)\right|^{2}+\left|\widetilde{\sigma}_{y}(t) \widehat{Y}(t)+\widetilde{\sigma}_{z}(t) \widehat{Z}(t)\right|^{2}\right) d t\right]
$$

independent of $\widehat{X}(\cdot)$, or have an estimate for

$$
\mathbb{E}\left[\left|\widetilde{h}_{\xi} \widehat{X}(T)\right|^{2}+\int_{0}^{T}\left|\widetilde{g}_{x}(t) \widehat{X}(t)\right|^{2} d t\right]
$$

independent of $(\widehat{Y}(\cdot), \widehat{Z}(\cdot))$. We now search conditions under which this is possible.

For any $\mathbf{b} \equiv\left(b_{1}, b_{2}, b_{3}\right), \boldsymbol{\sigma} \equiv\left(\sigma_{1}, \sigma_{2}, \sigma_{3}\right) \in \mathbb{R}^{n \times(n+2 m)}, \mathbf{g} \equiv\left(g_{1}, g_{2}, g_{3}\right) \in$ $\mathbb{R}^{m \times(n+2 m)}, \gamma \equiv\left(\gamma_{1}, \gamma_{2}\right) \in \mathbb{R}^{n \times(n+m)}$, and $\mathbf{h} \equiv\left(h_{1}, h_{2}\right) \in \mathbb{R}^{m \times(n+m)}$, with $b_{1}, \sigma_{1}, \gamma_{1} \in \mathbb{R}^{n \times n}, b_{2}, b_{3}, \sigma_{2}, \sigma_{3}, \gamma_{2} \in \mathbb{R}^{n \times m}, g_{1}, h_{1} \in \mathbb{R}^{m \times n}$, and $g_{2}, g_{3}, h_{2} \in \mathbb{R}^{m \times m}$, we denote

$$
\left\{\begin{array}{l}
\mathbf{A}=\left(\begin{array}{ll}
b_{1} & b_{2} \\
g_{1} & g_{2}
\end{array}\right)_{(n+m) \times(n+m)}, \quad \mathbf{B}=\left(\begin{array}{c}
b_{3} \\
g_{3}
\end{array}\right)_{(n+m) \times m}, \\
\mathbf{C}=\left(\begin{array}{cc}
\sigma_{1} & \sigma_{2} \\
0 & 0
\end{array}\right)_{(n+m) \times(n+m)}, \quad \mathbf{D}=\left(\begin{array}{c}
\sigma_{3} \\
0
\end{array}\right)_{(n+m) \times m}, \\
\boldsymbol{\Gamma}=\left(\begin{array}{cc}
\gamma_{1} & \gamma_{2} \\
0 & 0
\end{array}\right)_{(n+m) \times(n+m)}, \quad \mathbf{H}=\left(\begin{array}{cc}
0 & 0 \\
h_{1} & h_{2}
\end{array}\right)_{(n+m) \times(n+m)}, \\
J_{n}=\left(\begin{array}{cc}
I_{n} & 0 \\
0 & 0 I_{m}
\end{array}\right), \quad J_{m}=\left(\begin{array}{cc}
0 I_{n} & 0 \\
0 & I_{m}
\end{array}\right), \\
L_{n}=\left(\begin{array}{c}
I_{n} \\
0
\end{array}\right)_{(n+m) \times n}, \quad L_{m}=\left(\begin{array}{c}
0 \\
I_{m}
\end{array}\right)_{(n+m) \times m} .
\end{array}\right.
$$

Hereafter $I_{n}$ stands for the $(n \times n)$ identity matrix, and when the dimension is clear, we simply use $I$, omitting the subscript. Next, for any continuously differentiable function $P:[0, T] \rightarrow \mathcal{S}^{n+m}$, we define

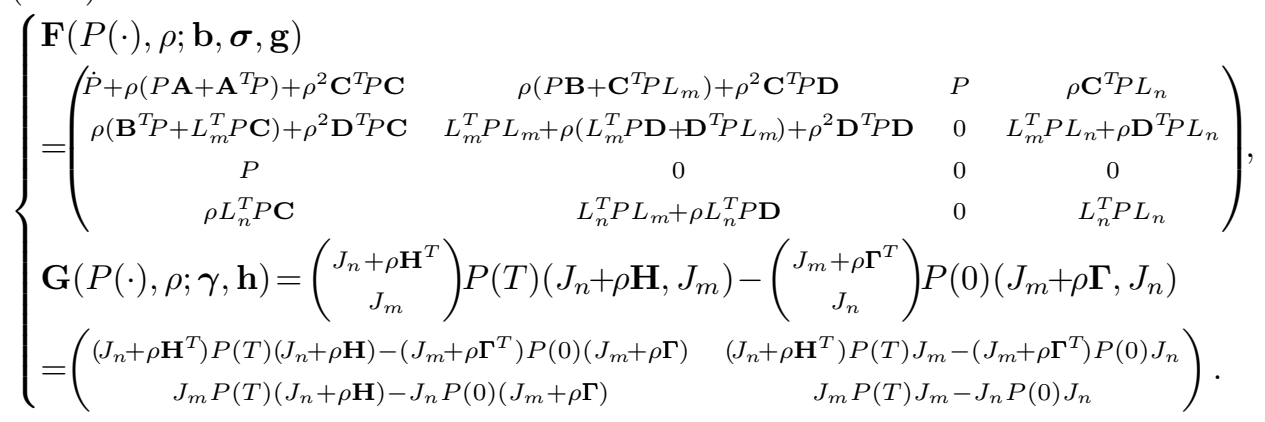


Clearly, $\mathbf{F}(P(\cdot), \rho ; \cdot): \mathbb{R}^{n \times(n+2 m)} \times \mathbb{R}^{n \times(n+2 m)} \times \mathbb{R}^{m \times(n+2 m)} \rightarrow \mathcal{S}^{2 n+4 m}$ and $\mathbf{G}(P(\cdot), \rho ; \cdot): \mathbb{R}^{n \times(n+m)} \times \mathbb{R}^{m \times(n+m)} \rightarrow \mathcal{S}^{2 n+2 m}$. The following lemma about the functions $\mathbf{F}(\cdot)$ and $\mathbf{G}(\cdot)$ defined above will be useful below.

Lemma 3.2. Let $P \equiv\left(\begin{array}{cc}P_{1} & \Psi^{T} \\ \Psi & P_{2}\end{array}\right)$ with $P_{1}:[0, T] \rightarrow \mathcal{S}^{n}, P_{2}:[0, T] \rightarrow \mathcal{S}^{m}$ and $\Psi:[0, T] \rightarrow \mathbb{R}^{m \times n}$ be continuously differentiable such that

$$
P_{1}(t)>0, \quad P_{2}(t)<0, \quad \dot{P}(t)>0, \quad t \in[0, T] .
$$

Then we have the following conclusions.

(i) There exist constants $\mu, K>0$ such that

$$
\mathbf{F}(P(\cdot), 0 ; \mathbf{b}, \boldsymbol{\sigma}, \mathbf{g}) \leq\left(\begin{array}{cc}
-\mu I_{n+2 m} & 0 \\
0 & K I_{2 n+m}
\end{array}\right)
$$

and

$$
\mathbf{G}(P(\cdot), 0 ; \gamma, \mathbf{h}) \geq\left(\begin{array}{cc}
\mu I_{n+m} & 0 \\
0 & -K I_{n+m}
\end{array}\right)
$$

(ii) Map $\rho \mapsto \mathbf{F}(P(\cdot), \rho ; \mathbf{b}, \boldsymbol{\sigma}, \mathbf{g})$ is convex and map $\rho \mapsto \mathbf{G}(P(\cdot), \rho ; \boldsymbol{\gamma}, \mathbf{h})$ is concave.

(iii) $\operatorname{Map}(\mathbf{b}, \boldsymbol{\sigma}, \mathbf{g}) \mapsto \mathbf{F}(P(\cdot), \rho ; \mathbf{b}, \boldsymbol{\sigma}, \mathbf{g})$ is convex and map $(\boldsymbol{\gamma}, \mathbf{h}) \mapsto \mathbf{G}(P(\cdot), \rho ; \boldsymbol{\gamma}, \mathbf{h})$ is concave. Consequently, if $(\mathbf{b}, \boldsymbol{\sigma}, \mathbf{g}, \boldsymbol{\gamma}, \mathbf{h}):[0,1] \rightarrow \mathbb{R}^{n \times(n+2 m)} \times \mathbb{R}^{n \times(n+2 m)} \times$ $\mathbb{R}^{m \times(n+2 m)} \times \mathbb{R}^{n \times(n+m)} \times \mathbb{R}^{m \times(n+m)}$ is continuous, then

$$
\begin{array}{r}
\mathbf{F}\left(P(\cdot), \rho ; \int_{0}^{1} \mathbf{b}(\alpha) d \alpha, \int_{0}^{1} \boldsymbol{\sigma}(\alpha) d \alpha, \int_{0}^{1} \mathbf{g}(\alpha) d \alpha\right) \\
\leq \int_{0}^{1} \mathbf{F}(P(\cdot), \rho ; \mathbf{b}(\alpha), \boldsymbol{\sigma}(\alpha), \mathbf{g}(\alpha)) d \alpha
\end{array}
$$

and

$$
\mathbf{G}\left(P(\cdot), \rho ; \int_{0}^{1} \gamma(\alpha) d \alpha, \int_{0}^{1} \mathbf{h}(\alpha) d \alpha\right) \geq \int_{0}^{1} \mathbf{G}(P(\cdot), \rho ; \gamma(\alpha), \mathbf{h}(\alpha)) d \alpha .
$$

Proof. From the definition, we have

$$
\mathbf{F}(P(\cdot), \rho ; \mathbf{b}, \boldsymbol{\sigma}, \mathbf{g})=\mathbf{F}_{0}(P(\cdot))+\rho \mathbf{F}_{1}(P(\cdot) ; \mathbf{b}, \boldsymbol{\sigma}, \mathbf{g})+\rho^{2} \mathbf{F}_{2}(P(\cdot) ; \mathbf{b}, \boldsymbol{\sigma}, \mathbf{g}),
$$

where

$$
\mathbf{F}_{0}(P(\cdot))=\mathbf{F}(P(\cdot), 0 ; \mathbf{b}, \boldsymbol{\sigma}, \mathbf{g})=\left(\begin{array}{cccc}
\dot{P} & 0 & P & 0 \\
0 & L_{m}^{T} P L_{m} & 0 & L_{m}^{T} P L_{n} \\
P & 0 & 0 & 0 \\
0 & L_{n}^{T} P L_{m} & 0 & L_{n}^{T} P L_{n}
\end{array}\right)=\left(\begin{array}{cccc}
\dot{P} & 0 & P & 0 \\
0 & P_{2} & 0 & \Psi \\
P & 0 & 0 & 0 \\
0 & \Psi^{T} & 0 & P_{1}
\end{array}\right)
$$

is independent of $(\mathbf{b}, \boldsymbol{\sigma}, \mathbf{g})$, satisfying (3.25) by (3.24),

$$
\mathbf{F}_{1}(P(\cdot) ; \mathbf{b}, \boldsymbol{\sigma}, \mathbf{g})=\left(\begin{array}{cccc}
P \mathbf{A}+\mathbf{A}^{T} P & P \mathbf{B}+\mathbf{C}^{T} P L_{m} & 0 & \mathbf{C}^{T} P L_{n} \\
\mathbf{B}^{T} P+L_{m}^{T} P \mathbf{C} & L_{m}^{T} P \mathbf{D}+\mathbf{D}^{T} P L_{m} & 0 & \mathbf{D}^{T} P L_{n} \\
0 & 0 & 0 & 0 \\
L_{n}^{T} P \mathbf{C} & L_{n}^{T} P \mathbf{D} & 0 & 0
\end{array}\right)
$$


is linear in $(\mathbf{b}, \boldsymbol{\sigma}, \mathbf{g})$, and

$$
\mathbf{F}_{2}(P(\cdot) ; \mathbf{b}, \boldsymbol{\sigma}, \mathbf{g})=\left(\begin{array}{cccc}
\mathbf{C}^{T} P \mathbf{C} & \mathbf{C}^{T} P \mathbf{D} & 0 & 0 \\
\mathbf{D}^{T} P \mathbf{C} & \mathbf{D}^{T} P \mathbf{D} & 0 & 0 \\
0 & 0 & 0 & 0 \\
0 & 0 & 0 & 0
\end{array}\right)=\left(\begin{array}{cc}
\boldsymbol{\sigma}^{T} P_{1}(t) \boldsymbol{\sigma} & 0 \\
0 & 0
\end{array}\right) \geq 0 .
$$

Thus, $\rho \mapsto \mathbf{F}(P(\cdot), \rho ; \mathbf{b}, \boldsymbol{\sigma}, \mathbf{g})$ and $(\mathbf{b}, \boldsymbol{\sigma}, \mathbf{g}) \mapsto \mathbf{F}(P(\cdot), \rho ; \mathbf{b}, \boldsymbol{\sigma}, \mathbf{g})$ are convex, and (3.27) follows. Similarly, we have

$$
\mathbf{G}(P(\cdot), \rho ; \boldsymbol{\gamma}, \mathbf{h})=\mathbf{G}_{0}(P(\cdot))+\rho \mathbf{G}_{1}(P(\cdot) ; \boldsymbol{\gamma}, \mathbf{h})+\rho^{2} \mathbf{G}_{2}(P(\cdot) ; \boldsymbol{\gamma}, \mathbf{h}),
$$

where

$$
\begin{aligned}
& \mathbf{G}_{0}(P(\cdot))=\mathbf{G}(P(\cdot), 0 ; \boldsymbol{\gamma}, \mathbf{h}) \\
& =\left(\begin{array}{ll}
J_{n} P(T) J_{n}-J_{m} P(0) J_{m} & J_{n} P(T) J_{m}-J_{m} P(0) J_{n} \\
J_{m} P(T) J_{n}-J_{n} P(0) J_{m} & J_{m} P(T) J_{m}-J_{n} P(0) J_{n}
\end{array}\right)=\left(\begin{array}{cccc}
P_{1}(T) & 0 & 0 & \Psi(T)^{T} \\
0 & -P_{2}(0) & -\Psi(0) & 0 \\
0 & -\Psi(0)^{T} & -P_{1}(0) & 0 \\
\Psi(T) & 0 & 0 & P_{2}(T)
\end{array}\right)
\end{aligned}
$$

is independent of $(\boldsymbol{\gamma}, \mathbf{h})$, satisfying (3.26) by (3.24),

$$
\mathbf{G}_{1}(P(\cdot) ; \boldsymbol{\gamma}, \mathbf{h})=\left(\begin{array}{cc}
J_{n} P(T) \mathbf{H}^{T}+\mathbf{H}^{T} P(T) J_{n}-\left(J_{m} P(0) \Gamma+\Gamma^{T} P(0) J_{m}\right. & \mathbf{H}^{T} P(T) J_{m}-\Gamma^{T} P(0) J_{n} \\
J_{m} P(T) \mathbf{H}-J_{n} P(0) \Gamma & 0
\end{array}\right)
$$

is linear in $(\boldsymbol{\gamma}, \mathbf{h})$, and

$$
\mathbf{G}_{2}(P(\cdot) ; \boldsymbol{\gamma}, \mathbf{h})=\left(\begin{array}{cc}
\mathbf{H}^{T} P(T) \mathbf{H}-\Gamma^{T} P(0) \Gamma & 0 \\
0 & 0
\end{array}\right)=\left(\begin{array}{cc}
\mathbf{h}^{T} P_{2}(T) \mathbf{h}-\boldsymbol{\gamma}^{T} P_{1}(0) \boldsymbol{\gamma} & 0 \\
0 & 0
\end{array}\right) \leq 0,
$$

which gives the concavity of $\rho \mapsto \mathbf{G}(P(\cdot), \rho ; \boldsymbol{\gamma}, \mathbf{h})$ and $(\boldsymbol{\gamma}, \mathbf{h}) \mapsto \mathbf{G}(P(\cdot), \rho ; \boldsymbol{\gamma}, \mathbf{h})$, and (3.28) follows immediately.

Next, for $(b, \sigma, g, \gamma, h) \in \mathcal{G}^{2}$, denote

$$
\left\{\begin{array}{l}
\mathbf{b}(t, \theta)=b_{\theta}(t, \theta) \equiv\left(b_{x}(t, \theta), b_{y}(t, \theta), b_{z}(t, \theta)\right) \\
\boldsymbol{\sigma}(t, \theta)=\sigma_{\theta}(t, \theta) \equiv\left(\sigma_{x}(t, \theta), \sigma_{y}(t, \theta), \sigma_{z}(t, \theta)\right) \\
\mathbf{g}(t, \theta)=g_{\theta}(t, \theta) \equiv\left(g_{x}(t, \theta), g_{y}(t, \theta), g_{z}(t, \theta)\right) \\
\gamma(\xi, y)=\left(\gamma_{\xi}(\xi, y), \gamma_{y}(\xi, y)\right), \quad \mathbf{h}(\xi, y)=\left(h_{\xi}(\xi, y), h_{y}(\xi, y)\right)
\end{array}\right.
$$

for any $(t, \theta) \in[0, T] \times \mathbb{M}, \xi \in \mathcal{X}^{2}$ (and $\omega \in \Omega$ which has been suppressed). Note that for given $(\xi, y) \in \mathcal{X}_{n}^{2} \times \mathbb{R}^{m}, \gamma_{\xi}(\xi, y): \mathcal{X}_{n}^{2} \rightarrow\left(\mathcal{X}_{n}^{2}\right)^{*} \equiv \mathcal{X}_{n}^{2}, \gamma_{y}(\xi, y): \mathbb{R}^{m} \rightarrow \mathbb{R}^{n}$, $h_{\xi}(\xi, y): \mathcal{X}_{n}^{2} \rightarrow \mathcal{X}_{m}^{2}$, and $h_{y}(\xi, y): \mathbb{R}^{m} \rightarrow \mathcal{X}_{m}^{2}$ are all linear bounded operators, with the operator norms all uniformly bounded. Note that since $\mathcal{X}_{n}^{2} \equiv\left[L_{\mathcal{F}_{T}}^{2}(\Omega ; \mathbb{R})\right]^{n}$, in the above, we regard $\gamma_{\xi}(\xi, y)$ as an $\mathbb{R}^{n}$-valued linear functional (meaning that it consists of $n$ components and each of them is a linear functional). A similar result applies to $h_{\xi}(\xi, y)$ and $h_{y}(\xi, y)$.

Now, for any continuously differentiable function $P:[0, T] \rightarrow \mathcal{S}^{n+m}$, we define

$$
\left\{\begin{array}{l}
\mathbf{F}(P(\cdot), \rho ; t, \theta)=\mathbf{F}(P(\cdot), \rho ; \mathbf{b}(t, \theta), \boldsymbol{\sigma}(t, \theta), \mathbf{g}(t, \theta)), \\
\mathbf{G}(P(\cdot), \rho ; \xi, y)=\mathbf{G}(P(\cdot), \rho ; \boldsymbol{\gamma}(\xi, y), \mathbf{h}(\xi, y)) .
\end{array}\right.
$$


Definition 3.3. Let $P \equiv\left(\begin{array}{cc}P_{1} & \Psi^{T} \\ \Psi & P_{2}\end{array}\right):[0, T] \rightarrow \mathcal{S}^{n+m}$ be continuously differentiable such that (3.24) holds.

(i) $P(\cdot)$ is called a type (I) Lyapunov operator of the generator $(b, \sigma, g, \gamma, h)$ if there exist constants $\mu, K>0$ such that

$$
\left\{\begin{array}{l}
\mathbf{F}(P(\cdot), 1 ; t, \theta) \leq\left(\begin{array}{ccc}
-\mu\left\{g_{x}^{T} g_{x}\right\}_{\infty} & 0 & 0 \\
0 & 0 I_{2 m} & 0 \\
0 & 0 & K I_{2 n+m}
\end{array}\right) \\
\mathbf{G}(P(\cdot), 1 ; \xi, y) \geq\left(\begin{array}{cc}
\mu\left\|h_{x}\right\|_{\infty}^{2} J_{n} & 0 \\
0 & -K I_{n+m}
\end{array}\right) .
\end{array}\right.
$$

(ii) $P(\cdot)$ is called a type (II) Lyapunov operator of the generator $(b, \sigma, g, \gamma, h)$ if there exist constants $\mu, K>0$ such that

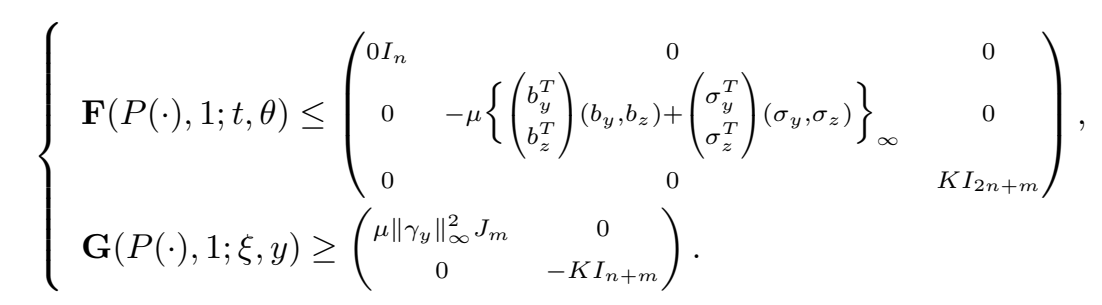

(iii) $P(\cdot)$ is called a type (III) Lyapunov operator of the generator $(b, \sigma, g, \gamma, h)$ if there exist constants $\mu, K>0$ such that

$$
\left\{\begin{array}{l}
\mathbf{F}(P(\cdot), 1 ; t, \theta) \leq\left(\begin{array}{cc}
-\mu I_{n+2 m} & 0 \\
0 & K I_{2 n+m}
\end{array}\right) \\
\mathbf{G}(P(\cdot), 1 ; \xi, y) \geq\left(\begin{array}{cc}
\mu I_{n+m} & 0 \\
0 & -K I_{n+m}
\end{array}\right)
\end{array}\right.
$$

Before going further, let us make a comment. Suppose the generator $(b, \sigma, g, \gamma, h)$ $\in \mathcal{G}^{2}$ admits a type (III) Lyapunov operator $P(\cdot)$ and $\Theta(\cdot) \in \mathcal{M}^{2}[0, T]$ is an adapted solution of FBSDE (1.1), and let $(\bar{b}, \bar{\sigma}, \bar{g}, \bar{\gamma}, \bar{h})=0$ (thus $\bar{\Theta}(\cdot)=0$ is an adapted solution of the corresponding (1.1)). Then we can show that (see the proof of Proposition 3.4)

$$
\begin{aligned}
& \mathbb{E}\left\{\left\langle P(T)\left(\begin{array}{c}
X(T) \\
Y(T)
\end{array}\right),\left(\begin{array}{c}
X(T) \\
Y(T)
\end{array}\right)\right\rangle\right\}-\left\langle P(0)\left(\begin{array}{c}
X(0) \\
Y(0)
\end{array}\right),\left(\begin{array}{c}
X(0) \\
Y(0)
\end{array}\right)\right\rangle \\
& \leq \mathbb{E} \int_{0}^{T}\left\{-\mu\left[|X(t)|^{2}+|Y(t)|^{2}+|Z(t)|^{2}\right]\right. \\
& \left.+K\left[|b(t, 0)|^{2}+|\sigma(t, 0)|^{2}+|g(t, 0)|^{2}\right]\right\} d t .
\end{aligned}
$$

Therefore, $(t, x, y) \mapsto\left\langle P(t)\left(\begin{array}{l}x \\ y\end{array}\right),\left(\begin{array}{l}x \\ y\end{array}\right)\right\rangle$ looks like a Lypunov function. Due to this, we borrow the name "Lyapunov operator" for $P(\cdot)$. But, we point out that $P(\cdot)$ is not even positive semi-definite!

From the definition, we see that if $P(\cdot)$ is a type (III) Lyapunov operator of $(b, \sigma, g, \gamma, h)$, then it is both a type (I) and a type (II) Lyapunov operator for the given generator. Note that since $P(\cdot) \mapsto \mathbf{F}(P(\cdot), \rho ; t, \theta)$ and $P(\cdot) \mapsto \mathbf{G}(P(\cdot), \rho ; \xi, y)$ 
are linear, the set of all type (I) (resp. type (II) and type (III)) Lyapunov operators of a given generator $(b, \sigma, g, \gamma, h) \in \mathcal{G}^{2}$ is a convex cone. In particular, if $P(\cdot)$ is a type (I) (resp. type (II), type (III)) Lyapunov operator of the generator $(b, \sigma, g, \gamma, h)$, so is $\nu P(\cdot)$ for any constant $\nu>0$.

Different from the notion of bridge introduced in [28, the Lyapunov operator introduced here has the following features: (i) Map $\gamma$ appears, which depends on $(\xi, y)$, but map $h$ is allowed to depend on $y$ (these make the existence of Lyapunov operators more difficult); (ii) $P(\cdot)$ is defined through the matrix-valued random fields associated with the single generator $(b, \sigma, g, \gamma, h)$ (defined by (3.22) ), which will be easier to use in applications. We note that the results obtained in [28] with the notion of a bridge did not fully cover the results of [23], whereas, the results presented in the current paper do fully cover those found in [23.

The following result gives the (uniform) stability of adapted solutions to FBSDE (3.7) when the generator $(b, \sigma, g, \gamma, h)$ admits a Lyapunov operator.

Proposition 3.4. Let $(b, \sigma, g, \gamma, h) \in \mathcal{G}^{2}$ admit a Lyapunov operator $P(\cdot)$ of either type (I) or (II) and satisfy both (3.15) and (3.18). For any $\rho \in[0,1]$, let $\Theta_{\rho}(\cdot) \equiv\left(X_{\rho}(\cdot), Y_{\rho}(\cdot), Z_{\rho}(\cdot)\right) \in \mathcal{M}^{2}[0, T]$ be an adapted solution of FBSDE (3.7) with some $\left(b_{0}(\cdot), \sigma_{0}(\cdot), g_{0}(\cdot), \gamma_{0}, h_{0}\right) \in \mathcal{G}_{0}^{2}$, and let $\bar{\Theta}_{\rho}(\cdot) \equiv\left(\bar{X}_{\rho}(\cdot), \bar{Y}_{\rho}(\cdot), \bar{Z}_{\rho}(\cdot)\right) \in$ $\mathcal{M}^{2}[0, T]$ be an adapted solution of FBSDE (3.7) corresponding to another generator $(\bar{b}, \bar{\sigma}, \bar{g}, \bar{\gamma}, \bar{h}) \in \mathcal{G}^{2}$ and some $\left(\bar{b}_{0}(\cdot), \bar{\sigma}_{0}(\cdot), \bar{g}_{0}(\cdot), \bar{\gamma}_{0}, \bar{h}_{0}\right) \in \mathcal{G}_{0}^{2}$. Then

$$
\begin{aligned}
& \left\|\bar{\Theta}_{\rho}(\cdot)-\Theta_{\rho}(\cdot)\right\|_{\mathcal{M}^{2}[0, T]} \\
& \equiv \mathbb{E}\left\{\sup _{t \in[0, T]}\left|\bar{X}_{\rho}(t)-X_{\rho}(t)\right|^{2}+\sup _{t \in[0, T]}\left|\bar{Y}_{\rho}(t)-Y_{\rho}(t)\right|^{2}+\int_{0}^{T}\left|\bar{Z}_{\rho}(t)-Z_{\rho}(t)\right|^{2} d t\right\} \\
& \leq K \mathbb{E}\left\{\left|\bar{\gamma}_{0}-\gamma_{0}\right|^{2}+\left|\bar{h}_{0}-h_{0}\right|^{2}+\left|\bar{\gamma}_{(}\left(\bar{X}_{\rho}(T), \bar{Y}_{\rho}(0)\right)-\gamma\left(\bar{X}_{\rho}(T), \bar{Y}_{\rho}(0)\right)\right|^{2}\right. \\
& \quad+\left|\bar{h}\left(\bar{X}_{\rho}(0), \bar{Y}_{\rho}(T)\right)-h\left(\bar{X}_{\rho}(0), \bar{Y}_{\rho}(T)\right)\right|^{2}+\int_{0}^{T}\left(\left|\bar{b}_{0}(t)-b_{0}(t)\right|^{2}\right. \\
& \quad+\left|\bar{\sigma}_{0}(t)-\sigma_{0}(t)\right|^{2}+\left|\bar{g}_{0}(t)-g_{0}(t)\right|^{2}+\left|\bar{b}\left(t, \bar{\Theta}_{\rho}(t)\right)-b\left(t, \bar{\Theta}_{\rho}(t)\right)\right|^{2} \\
& \left.\left.\quad+\left|\bar{\sigma}\left(t, \bar{\Theta}_{\rho}(t)\right)-\sigma\left(t, \bar{\Theta}_{\rho}(t)\right)\right|^{2}+\left|\bar{g}\left(t, \bar{\Theta}_{\rho}(t)\right)-g\left(t, \bar{\Theta}_{\rho}(t)\right)\right|^{2}\right) d t\right\},
\end{aligned}
$$

uniformly in $\rho \in[0,1]$. In particular, if $(\bar{b}, \bar{\sigma}, \bar{g}, \bar{\gamma}, \bar{h})=(b, \sigma, g, \gamma, h)$, then

$$
\begin{aligned}
& \left\|\bar{\Theta}_{\rho}(\cdot)-\Theta_{\rho}(\cdot)\right\|_{\mathcal{M}^{2}[0, T]}^{2} \\
& \equiv \mathbb{E}\left\{\sup _{t \in[0, T]}\left|\bar{X}_{\rho}(t)-X_{\rho}(t)\right|^{2}+\sup _{t \in[0, T]}\left|\bar{Y}_{\rho}(t)-Y_{\rho}(t)\right|^{2}+\int_{0}^{T}\left|\bar{Z}_{\rho}(t)-Z_{\rho}(t)\right|^{2} d t\right\} \\
& \leq K \mathbb{E}\left\{\left|\bar{\gamma}_{0}-\gamma_{0}\right|^{2}+\left|\bar{h}_{0}-h_{0}\right|^{2}\right. \\
& \left.\quad \quad \quad \int_{0}^{T}\left(\left|\bar{b}_{0}(t)-b_{0}(t)\right|^{2}+\left|\bar{\sigma}_{0}(t)-\sigma_{0}(t)\right|^{2}+\left|\bar{g}_{0}(t)-g_{0}(t)\right|^{2}\right) d t\right\},
\end{aligned}
$$

uniformly in $\rho \in[0,1]$.

Moreover, if $P(\cdot)$ is a type (III) Lyapunov operator of the generator $(b, \sigma, g, \gamma, h)$, then the above holds without conditions (3.15) and (3.18). 
Proof. It suffices to consider the case that all the involved functions in (3.8) are bounded. For the general case, we can use standard approximation. We adopt the notation in (3.9)-(3.13), and (3.29). Similarly, we denote

$$
\left\{\begin{array}{rr}
\tilde{f}=\int_{0}^{1} f^{\alpha} d \alpha, \quad f^{\alpha}=f\left(X_{\rho}(T)+\alpha \widehat{X}(T), Y_{\rho}(0)+\alpha \widehat{Y}(0)\right), \\
\text { for } f(\cdot)=\mathbf{g}(\cdot), \mathbf{h}(\cdot), \\
\widetilde{f}(t)=\int_{0}^{1} f^{\alpha}(t) d \alpha, \quad f^{\alpha}(t)=f\left(t, \Theta_{\rho}(t)+\alpha \widehat{\Theta}(t)\right), \\
\text { for } f(\cdot)=\mathbf{b}(\cdot), \boldsymbol{\sigma}(\cdot), \mathbf{g}(\cdot) .
\end{array}\right.
$$

Now, applying Itô's formula to $\left\langle P(\cdot)\left(\begin{array}{c}\widehat{X}(\cdot) \\ \widehat{Y}(\cdot)\end{array}\right),\left(\begin{array}{c}\widehat{X}(\cdot) \\ \widehat{Y}(\cdot)\end{array}\right)\right\rangle$, we get

$$
\begin{gathered}
\mathbb{E}\left\langle P(T)\left(\begin{array}{c}
\widehat{X}(T) \\
\rho \widehat{h}+\delta h_{0}
\end{array}\right),\left(\begin{array}{c}
\widehat{X}(T) \\
\rho \widehat{h}+\delta h_{0}
\end{array}\right)\right\rangle-\left\langle P(0)\left(\begin{array}{c}
\rho \widehat{\gamma}+\delta \gamma_{0} \\
\widehat{Y}(0)
\end{array}\right),\left(\begin{array}{c}
\rho \widehat{\gamma}+\delta \gamma_{0} \\
\widehat{Y}(0)
\end{array}\right)\right\rangle \\
=\mathbb{E} \int_{0}^{T}\left\{\left\langle\dot{P}(t)\left(\begin{array}{c}
\widehat{X}(t) \\
\widehat{Y}(t)
\end{array}\right),\left(\begin{array}{c}
\widehat{X}(t) \\
\widehat{Y}(t)
\end{array}\right)\right\rangle+2\left\langle P(t)\left(\begin{array}{c}
\widehat{X}(t) \\
\widehat{Y}(t)
\end{array}\right),\left(\begin{array}{c}
\rho \widehat{b}(t)+\delta b_{0}(t) \\
\rho \widehat{g}(t)+\delta g_{0}(t)
\end{array}\right)\right\rangle\right. \\
\left.+\left\langle P(t)\left(\begin{array}{c}
\rho \widehat{\sigma}(t)+\delta \sigma_{0}(t) \\
\widehat{Z}(t)
\end{array}\right),\left(\begin{array}{c}
\rho \widehat{\sigma}(t)+\delta \sigma_{0}(t) \\
\widehat{Z}(t)
\end{array}\right)\right\rangle\right\} d t .
\end{gathered}
$$

We first assume that $P(\cdot)$ is a type (I) Lyapunov operator. Let us look at each side of (3.37) separately. Observe

$$
\begin{aligned}
& \left\langle P(T)\left(\begin{array}{c}
\widehat{X}(T) \\
\rho \hat{h}+\delta h_{0}
\end{array}\right),\left(\begin{array}{c}
\widehat{X}(T) \\
\rho \widehat{h}+\delta h_{0}
\end{array}\right)\right\rangle-\left\langle P(0)\left(\begin{array}{c}
\rho \widehat{\gamma}+\delta \gamma_{0} \\
\widehat{Y}(0)
\end{array}\right),\left(\begin{array}{c}
\rho \widehat{\gamma}+\delta \gamma_{0} \\
\widehat{Y}(0)
\end{array}\right)\right\rangle \\
& =\left\langle P(T)\left(\begin{array}{c}
\widehat{X}(T) \\
\rho\left[\widetilde{h}_{\xi} \widehat{X}(T)+\widetilde{h}_{y} \widehat{Y}(0)+\delta h\right]+\delta h_{0}
\end{array}\right),\left(\begin{array}{c}
\widehat{X}(T) \\
\rho\left[\widetilde{h}_{\xi} \widehat{X}(T)+\widetilde{h}_{y} \widehat{Y}(0)+\delta h\right]+\delta h_{0}
\end{array}\right)\right\rangle \\
& \quad-\left\langle P(0)\left(\begin{array}{c}
\rho\left[\widetilde{\gamma}_{\xi} \widehat{X}(T)+\widetilde{\gamma}_{\widehat{Y}} \widehat{Y}(0)+\delta \gamma\right]+\delta \gamma_{0} \\
\widehat{Y}(0)
\end{array}\right),\left(\begin{array}{c}
\left.\rho \widetilde{\gamma}_{\xi} \widehat{X}(T)+\tilde{\gamma}_{y} \widehat{Y}(0)+\delta \gamma\right]+\delta \gamma_{0} \\
\widehat{Y}(0)
\end{array}\right)\right\rangle \\
& =\left\langle\widetilde{\mathbf{G}}(P(\cdot), \rho)\left(\begin{array}{c}
\widehat{X}(T) \\
\widehat{Y}(0) \\
\rho \delta \gamma+\delta \gamma_{0} \\
\rho \delta h+\delta h_{0}
\end{array}\right),\left(\begin{array}{c}
\widehat{X}(T) \\
\widehat{Y}(0) \\
\rho \delta \gamma+\delta \gamma_{0} \\
\rho \delta h+\delta h_{0}
\end{array}\right)\right\rangle,
\end{aligned}
$$

where, making use of Lemma 3.2,

$$
\begin{aligned}
\widetilde{\mathbf{G}}(P(\cdot), \rho) & =\mathbf{G}\left(P(\cdot), \rho ; \int_{0}^{1} \widetilde{\boldsymbol{\gamma}}^{\alpha} d \alpha, \int_{0}^{1} \widetilde{\mathbf{h}}^{\alpha} d \alpha\right) \geq \int_{0}^{1} \mathbf{G}\left(P(\cdot), \rho ; \widetilde{\boldsymbol{\gamma}}^{\alpha}, \widetilde{\mathbf{h}}^{\alpha}\right) d \alpha \\
& \geq\left(\begin{array}{cc}
\mu\left\|h_{\xi}\right\|_{\infty}^{2} J_{n} & 0 \\
0 & -K I_{n+m}
\end{array}\right) \geq\left(\begin{array}{ccc}
\mu \widetilde{h}_{\xi}^{*} \widetilde{h}_{\xi} & 0 & 0 \\
0 & 0 I_{m} & 0 \\
0 & 0 & -K I_{n+m}
\end{array}\right) .
\end{aligned}
$$

Consequently,

$$
\begin{aligned}
& \left\langle P(T)\left(\begin{array}{c}
\widehat{X}(T) \\
\rho \widehat{h}+\delta h_{0}
\end{array}\right),\left(\begin{array}{c}
\widehat{X}(T) \\
\rho \widehat{h}+\delta h_{0}
\end{array}\right)\right\rangle-\left\langle P(0)\left(\begin{array}{c}
\rho \widehat{\gamma}+\delta \gamma_{0} \\
\widehat{Y}(0)
\end{array}\right),\left(\begin{array}{c}
\rho \widehat{\gamma}+\delta \gamma_{0} \\
\widehat{Y}(0)
\end{array}\right)\right\rangle \\
& \geq \mu\left|\widetilde{h}_{\xi} \widehat{X}(T)\right|^{2}-K\left[|\delta \gamma|^{2}+\left|\delta \gamma_{0}\right|^{2}+|\delta h|^{2}+\left|\delta h_{0}\right|^{2}\right] .
\end{aligned}
$$


On the other hand, for the right hand side of (3.37), we have

$$
\begin{aligned}
& \left\langle\dot{P}\left(\begin{array}{c}
\widehat{X} \\
\widehat{Y}
\end{array}\right),\left(\begin{array}{c}
\widehat{X} \\
\widehat{Y}
\end{array}\right)\right\rangle+2\left\langle P\left(\begin{array}{c}
\rho \hat{b}+\delta b_{0} \\
\rho \widehat{g}+\delta g_{0}
\end{array}\right),\left(\begin{array}{c}
\widehat{X} \\
\widehat{Y}
\end{array}\right)\right\rangle+\left\langle P\left(\begin{array}{c}
\rho \widehat{\sigma}+\delta \sigma_{0} \\
\widehat{Z}
\end{array}\right),\left(\begin{array}{c}
\rho \widehat{\sigma}+\delta \sigma_{0} \\
\widehat{Z}
\end{array}\right)\right\rangle \\
& =\left\langle\dot{P}\left(\begin{array}{c}
\widehat{X} \\
\widehat{Y}
\end{array}\right),\left(\begin{array}{c}
\widehat{X} \\
\widehat{Y}
\end{array}\right)\right\rangle+2\left\langle P\left(\begin{array}{c}
\rho\left(\widetilde{b}_{\theta} \widehat{\Theta}+\delta b\right)+\delta b_{0} \\
\rho\left(\widetilde{g}_{\theta} \widehat{\Theta}+\delta g\right)+\delta g_{0}
\end{array}\right),\left(\begin{array}{c}
\widehat{X} \\
\widehat{Y}
\end{array}\right)\right\rangle \\
& +\left\langle P\left(\begin{array}{c}
\rho\left(\widetilde{\sigma}_{\theta} \widehat{\Theta}+\delta \sigma\right)+\delta \sigma_{0} \\
\widehat{Z}
\end{array}\right),\left(\begin{array}{c}
\rho\left(\widetilde{\sigma}_{\theta} \widehat{\Theta}+\delta \sigma\right)+\delta \sigma_{0} \\
\widehat{Z}
\end{array}\right)\right\rangle \\
& =\left\langle\widetilde{\mathbf{F}}(P(\cdot), \rho ; t)\left(\begin{array}{c}
\widehat{X} \\
\widehat{Y} \\
\widehat{Z} \\
\rho \delta b+\delta b_{0} \\
\rho \delta g+\delta g_{0} \\
\rho \delta \sigma+\delta \sigma_{0}
\end{array}\right),\left(\begin{array}{c}
\widehat{X} \\
\widehat{Y} \\
\widehat{Z} \\
\rho \delta b+\delta b_{0} \\
\rho \delta g+\delta g_{0} \\
\rho \delta \sigma+\delta \sigma_{0}
\end{array}\right)\right\rangle,
\end{aligned}
$$

where, making use of Lemma 3.2 again,

$$
\begin{aligned}
& \widetilde{\mathbf{F}}(P(\cdot), \rho ; t)=\mathbf{F}\left(P(\cdot), \rho ; \int_{0}^{1} \widetilde{\mathbf{b}}^{\alpha}(t) d \alpha, \int_{0}^{1} \widetilde{\boldsymbol{\sigma}}^{\alpha}(t) d \alpha, \int_{0}^{1} \widetilde{\mathbf{g}}^{\alpha}(t) d \alpha\right) \\
& \leq \int_{0}^{1} \mathbf{F}\left(P(\cdot), \rho ; \widetilde{\mathbf{b}}^{\alpha}(t), \widetilde{\boldsymbol{\sigma}}^{\alpha}(t), \widetilde{\mathbf{g}}^{\alpha}(t)\right) d \alpha \leq\left(\begin{array}{ccc}
-\mu\left\{g_{x}^{T} g_{x}\right\}_{\infty} & 0 & 0 \\
0 & 0 I_{2 m} & 0 \\
0 & 0 & K I_{2 n+m}
\end{array}\right) \\
& \leq\left(\begin{array}{ccc}
-\mu \widetilde{g}_{x}^{T} \widetilde{g}_{x} & 0 & 0 \\
0 & 0 I_{2 m} & 0 \\
0 & 0 & K I_{2 n+m}
\end{array}\right) .
\end{aligned}
$$

This leads to

$$
\begin{aligned}
& \left\langle\dot{P}\left(\begin{array}{c}
\widehat{X} \\
\widehat{Y}
\end{array}\right),\left(\begin{array}{c}
\widehat{X} \\
\widehat{Y}
\end{array}\right)\right\rangle+2\left\langle P\left(\begin{array}{c}
\rho \widehat{b}+\delta b_{0} \\
\rho \widehat{g}+\delta g_{0}
\end{array}\right),\left(\begin{array}{c}
\widehat{X} \\
\widehat{Y}
\end{array}\right)\right\rangle+\left\langle P\left(\begin{array}{c}
\rho \widehat{\sigma}+\delta \sigma_{0} \\
\widehat{Z}
\end{array}\right),\left(\begin{array}{c}
\rho \widehat{\sigma}+\delta \sigma_{0} \\
\widehat{Z}
\end{array}\right)\right\rangle \\
& \leq-\mu\left|\widetilde{g}_{x} \widehat{X}\right|^{2}+K\left(|\delta b|^{2}+\left|\delta b_{0}\right|^{2}+|\delta \sigma|^{2}+\left|\delta \sigma_{0}\right|^{2}+|\delta g|^{2}+\left|\delta g_{0}\right|^{2}\right) .
\end{aligned}
$$

Hence, we obtain

$$
\begin{aligned}
\mu \mathbb{E}\left\{\left|\widetilde{h}_{\xi} \widehat{X}(T)\right|^{2}+\right. & \left.\int_{0}^{T}\left|\widetilde{g}_{x} \widehat{X}(t)\right|^{2} d t\right\} \leq K \mathbb{E}\left\{|\delta \gamma|^{2}+\left|\delta \gamma_{0}\right|^{2}+|\delta h|^{2}+\left|\delta h_{0}\right|^{2}\right. \\
& \left.+\int_{0}^{T}\left[|\delta b|^{2}+\left|\delta b_{0}\right|^{2}+|\delta \sigma|^{2}+\left|\delta \sigma_{0}\right|^{2}+|\delta g|^{2}+\left|\delta g_{0}\right|^{2}\right] d t\right\} .
\end{aligned}
$$

Applying (iii) of Proposition 3.1, one has

$$
\begin{aligned}
&\|\widehat{\Theta}(\cdot)\|_{\mathcal{M}^{2}[0, T]}^{2} \equiv \mathbb{E}\left[\sup _{t \in[0, T]}|\widehat{X}(t)|^{2}+\sup _{t \in[0, T]}|\widehat{Y}(t)|^{2}+\int_{0}^{T}|\widehat{Z}(t)|^{2} d t\right] \\
& \leq K \mathbb{E}\left\{|\delta \gamma|^{2}+\left|\delta \gamma_{0}\right|^{2}+|\delta h|^{2}+\left|\delta h_{0}\right|^{2}\right. \\
&\left.\quad+\int_{0}^{T}\left[|\delta b|^{2}+\left|\delta b_{0}\right|^{2}+|\delta \sigma|^{2}+\left|\delta \sigma_{0}\right|^{2}+|\delta g|^{2}+\left|\delta g_{0}\right|^{2}\right] d t\right\} .
\end{aligned}
$$


Similarly we can show the conclusion for the case that $P(\cdot)$ is a type (II) Lyapunov operator. In the case that $P(\cdot)$ is a type (III) Lyapunov operator, we can obtain directly from (3.37) that

$$
\begin{aligned}
& \mathbb{E}\left\{|\widehat{X}(T)|^{2}+|\widehat{Y}(0)|^{2}+\int_{0}^{T}\left[|\widehat{X}(t)|^{2}+|\widehat{Y}(t)|^{2}+|\widehat{Z}(t)|^{2}\right] d t\right\} \\
& \leq K \mathbb{E}\left\{|\delta \gamma|^{2}+\left|\delta \gamma_{0}\right|^{2}+|\delta h|^{2}+\left|\delta h_{0}\right|^{2}\right. \\
& \left.\quad+\int_{0}^{T}\left[|\delta b|^{2}+\left|\delta b_{0}\right|^{2}+|\delta \sigma|^{2}+\left|\delta \sigma_{0}\right|^{2}+|\delta g|^{2}+\left|\delta g_{0}\right|^{2}\right] d t\right\} .
\end{aligned}
$$

Then (3.38) follows without conditions (3.15) and (3.18).

Note that in order to have (3.35), we only require that the generator $(b, \sigma, g, \gamma, h)$ admits a Lyapunov operator, and no such condition is required for the other generator $(\bar{b}, \bar{\sigma}, \bar{g}, \bar{\gamma}, \bar{h})$. The above proposition tells us that when $(b, \sigma, g, \gamma, h) \in \mathcal{G}^{2}$ admits a type (III) Lyapunov operator, or admits a type (I) or type (II) Lyapunov operator, and (3.15) and (3.18) hold, then the adapted solution is unique.

\section{WELL-POSEDNESS OF FBSDES}

We are now at the position to state and prove the following theorem concerning the well-posedness of FBSDE (1.1).

Theorem 4.1. For some $p \geq 2$, let $(b, \sigma, g, \gamma, h) \in \mathcal{G}^{p}$ satisfy (3.15) and (3.18), and admit a type (I) or (II) Lyapunov operator $P(\cdot)$. Then FBSDE (1.1) admits a unique adapted solution $\Theta(\cdot) \equiv(X(\cdot), Y(\cdot), Z(\cdot)) \in \mathcal{M}^{p}[0, T]$. Moreover, the following estimate holds:

$$
\begin{aligned}
& \|\Theta(\cdot)\|_{\mathcal{M}^{p}[0, T]}^{p} \equiv \mathbb{E}\left\{\sup _{t \in[0, T]}|X(t)|^{p}+\sup _{t \in[0, T]}|Y(t)|^{p}+\left(\int_{0}^{T}|Z(t)|^{2} d t\right)^{\frac{p}{2}}\right\} \\
& \leq K \mathbb{E}\left\{|\gamma(0,0)|^{p}+|h(0,0)|^{p}+\left(\int_{0}^{T}|b(t, 0)| d t\right)^{p}+\left(\int_{0}^{T}|g(t, 0)| d t\right)^{p}\right. \\
& \left.+\left(\int_{0}^{T}|\sigma(t, 0)|^{2} d t\right)^{\frac{p}{2}}\right\} .
\end{aligned}
$$

Furthermore, if $\bar{\Theta}(\cdot) \equiv(\bar{X}(\cdot), \bar{Y}(\cdot), \bar{Z}(\cdot)) \in \mathcal{M}^{p}[0, T]$ is an adapted solution of (1.1) corresponding to $(\bar{b}, \bar{\sigma}, \bar{g}, \bar{\gamma}, \bar{h}) \in \mathcal{G}^{p}$, then the following stability estimate holds:

$$
\begin{aligned}
& \|\bar{\Theta}(\cdot)-\Theta(\cdot)\|_{\mathcal{M}^{p}[0, T]}^{p} \\
& \equiv \mathbb{E}\left\{\sup _{t \in[0, T]}|\bar{X}(t)-X(t)|^{p}+\sup _{t \in[0, T]}|\bar{Y}(t)-Y(t)|^{p}+\left(\int_{0}^{T}|\bar{Z}(t)-Z(t)|^{2} d t\right)^{\frac{p}{2}}\right\} \\
& \leq K \mathbb{E}\left\{|\bar{\gamma}(\bar{X}(T), \bar{Y}(0))-\gamma(\bar{X}(T), \bar{Y}(0))|^{p}+|\bar{h}(\bar{X}(T), \bar{Y}(0))-h(\bar{X}(T), \bar{Y}(0))|^{p}\right. \\
& +\left(\int_{0}^{T} \bar{\phi}(t, \bar{\Theta}(t))-b(t, \bar{\Theta}(t)) \mid d t\right)^{p}+\left(\int_{0}^{T}|\bar{g}(t, \bar{\Theta}(t))-g(t, \bar{\Theta}(t))| d t\right)^{p} \\
& \left.+\left(\int_{0}^{T}|\bar{\sigma}(t, \bar{\Theta}(t))-\sigma(t, \bar{\Theta}(t))|^{2} d t\right)^{\frac{p}{2}}\right\} .
\end{aligned}
$$


If $P(\cdot)$ is a type (III) Lyapunov operator of $(b, \sigma, g, \gamma, h)$, then (3.15) and (3.18) are not necessary.

Note that in 28, a similar result was proved for the case $p=2,\left\|\gamma_{\xi}\right\|_{\infty}=$ $\left\|\gamma_{y}\right\|_{\infty}=\left\|h_{y}\right\|_{\infty}=0$. From the FBSDEs theory point of view, Theorem 4.1 is a very natural and desirable improvement of the result in [28. On the other hand, as we pointed out earlier, the $L^{p}$-estimate (for $p>2$ ) will be very crucial in the study of necessary conditions of optimal controls for FBSDEs.

The proof of the above theorem looks somehow similar to that given in [28]. However, some crucial modifications are necessary. The following lemma will play an interesting role in the proof of Theorem 4.1.

Lemma 4.1. Suppose $p \geq 2$ and the sequence $\Theta^{k}(\cdot) \in \mathcal{M}^{p}[0, T](k \geq 1)$ satisfies

$$
\begin{aligned}
\left\|\Theta^{k}(\cdot)\right\|_{\mathcal{M}^{p}[0, T]} \leq K, \quad k & \geq 1, \\
\lim _{k \rightarrow \infty}\left\|\Theta^{k}(\cdot)-\Theta(\cdot)\right\|_{\mathcal{M}^{2}[0, T]} & =0 .
\end{aligned}
$$

Then $\Theta(\cdot) \in \mathcal{M}^{p}[0, T]$.

Proof. Let $\Theta^{k}(\cdot)=\left(X^{k}(\cdot), Y^{k}(\cdot), Z^{k}(\cdot)\right)$ and $\Theta(\cdot)=(X(\cdot), Y(\cdot), Z(\cdot))$. According to our condition, we may assume that

$$
\lim _{k \rightarrow \infty} X^{k}(t, \omega)=X(t, \omega), \quad æ t \in[0, T], \text { a.s. } \omega \in \Omega .
$$

Also, for any $r>1$, we have

$$
\mathbb{E}\left[\int_{0}^{T}\left|X^{k}(t)\right|^{r} d t\right]^{\frac{p}{r}} \leq T^{\frac{p}{r}} \mathbb{E}\left[\sup _{t \in[0, T]}\left|X^{k}(t)\right|^{p}\right] \leq T^{\frac{p}{r}}\left\|\Theta^{k}(\cdot)\right\|_{\mathcal{M}^{p}[0, T]}^{p} \leq K,
$$

with $K$ independent of $r>1$. By Fatou's lemma, sending $k \rightarrow \infty$ in the above, we have

$$
\mathbb{E}\left[\int_{0}^{T}|X(t)|^{r} d t\right]^{\frac{p}{r}} \leq K, \quad \forall r>1 .
$$

Now, using Fatou's Lemma again, sending $r \rightarrow \infty$, we obtain

$$
\mathbb{E}\left[\sup _{t \in[0, T]}|X(t)|^{p}\right] \leq K .
$$

Similarly, we can show that

$$
\mathbb{E}\left[\sup _{t \in[0, T]}|Y(t)|^{p}\right] \leq K, \quad \mathbb{E}\left(\int_{0}^{T}|Z(t)|^{2} d t\right)^{\frac{p}{2}} \leq K .
$$

Thus $\Theta(\cdot) \in \mathcal{M}^{p}[0, T]$.

Proof of Theorem 4.1. For $p \geq 1$, let $\widetilde{\mathcal{G}}_{0}^{p}$ be the set of all 5 -tuples $\left(b_{0}, \sigma_{0}, g_{0}, \gamma_{0}, h_{0}\right) \in$ $\mathcal{G}_{0}^{p}$ satisfying the following:

$$
b_{0}(\cdot) \in L_{\mathbb{F}}^{p}\left(\Omega ; L^{2}\left(0, T ; \mathbb{R}^{n}\right)\right), \quad g_{0}(\cdot) \in L_{\mathbb{F}}^{p}\left(\Omega ; L^{2}\left(0, T ; \mathbb{R}^{m}\right)\right),
$$


and let $\widetilde{\mathcal{G}}^{p}$ be the set of all $(b, \sigma, g, \gamma, h) \in \mathcal{G}^{p}$ such that

$$
(b(\cdot, 0), \sigma(\cdot, 0), g(\cdot, 0), \gamma(0), h(0)) \in \widetilde{\mathcal{G}}_{0}^{p} .
$$

We first let $(b, \sigma, g, \gamma, h) \in \widetilde{\mathcal{G}}^{p}$ and take $\left(b_{0}, \sigma_{0}, g_{0}, \gamma_{0}, h_{0}\right) \in \widetilde{\mathcal{G}}_{0}^{p}$. Let $\rho \in[0,1)$. Suppose the following coupled FBSDE admits a unique adapted solution $\Theta_{\rho}(\cdot) \equiv$ $\left(X_{\rho}(\cdot), Y_{\rho}(\cdot), Z_{\rho}(\cdot)\right) \in \mathcal{M}^{p}[0, T]$ :

$$
\left\{\begin{array}{l}
d X_{\rho}(t)=\left[\rho b\left(t, \Theta_{\rho}(t)\right)+b_{0}(t)\right] d t+\left[\rho \sigma\left(t, \Theta_{\rho}(t)\right)+\sigma_{0}(t)\right] d W(t) \\
d Y_{\rho}(t)=\left[\rho g\left(t, \Theta_{\rho}(t)\right)+g_{0}(t)\right] d t+Z_{\rho}(t) d W(t) \\
X_{\rho}(0)=\rho \gamma\left(X_{\rho}(T), Y_{\rho}(0)\right)+\gamma_{0}, \quad Y_{\rho}(T)=\rho h\left(X_{\rho}(T), Y_{\rho}(0)\right)+h_{0}
\end{array}\right.
$$

and the following estimate holds:

$$
\begin{aligned}
\left\|\Theta_{\rho}(\cdot)\right\|_{\mathcal{M}^{p}[0, T]}^{p} \equiv \mathbb{E}\left\{\sup _{t \in[0, T]}\left|X_{\rho}(t)\right|^{p}+\sup _{t \in[0, T]}\left|Y_{\rho}(t)\right|^{p}+\left(\int_{0}^{T}\left|Z_{\rho}(t)\right|^{2} d t\right)^{\frac{p}{2}}\right\} \\
\leq K \mathbb{E}\left\{\left(|\gamma(0,0)|+\left|\gamma_{0}\right|\right)^{p}+\left(|h(0,0)|+\left|h_{0}\right|\right)^{p}+\left[\int_{0}^{T}\left(|b(t, 0)|+\left|b_{0}(t)\right|\right)^{2} d t\right]^{\frac{p}{2}}\right. \\
\left.+\left[\int_{0}^{T}\left(|\sigma(t, 0)|+\left|\sigma_{0}(t)\right|\right)^{2} d t\right]^{\frac{p}{2}}+\left[\int_{0}^{T}\left(|g(t, 0)|+\left|g_{0}(t)\right|\right)^{2} d t\right]^{\frac{p}{2}}\right\} .
\end{aligned}
$$

Clearly, the above holds if $\rho=0$. Now, let $\varepsilon>0$ such that $\rho+\varepsilon \in[0,1]$. Consider the following coupled FBSDE:

$$
\left\{\begin{aligned}
d X_{\rho+\varepsilon}(t)= & {\left[(\rho+\varepsilon) b\left(t, \Theta_{\rho+\varepsilon}(t)\right)+b_{0}(t)\right] d t } \\
& +\left[(\rho+\varepsilon) \sigma\left(t, \Theta_{\rho+\varepsilon}(t)\right)+\sigma_{0}(t)\right] d W(t), \\
d Y_{\rho+\varepsilon}(t)= & {\left[(\rho+\varepsilon) g\left(t, \Theta_{\rho+\varepsilon}(t)\right)+g_{0}(t)\right] d t+Z_{\rho+\varepsilon}(t) d W(t), } \\
X_{\rho+\varepsilon}(0)= & (\rho+\varepsilon) \gamma\left(X_{\rho+\varepsilon}(T), Y_{\rho+\varepsilon}(0)\right)+\gamma_{0}, \\
Y_{\rho+\varepsilon}(T)= & (\rho+\varepsilon) h\left(X_{\rho+\varepsilon}(T), Y_{\rho+\varepsilon}(0)\right)+h_{0} .
\end{aligned}\right.
$$

To obtain the (unique) solvability of the above FBSDE, we introduce the following sequence of FBSDEs:

$$
\left\{\begin{aligned}
& \Theta^{0}(t) \triangleq \triangleq \\
&\left.d X^{0}(t), Y^{0}(t), Z^{0}(t)\right) \equiv 0 \\
& \quad+\left[\rho b\left(t, \Theta^{k+1}(t)\right)+\varepsilon b\left(t, \Theta^{k}(t)\right)+b_{0}(t)\right] d t \\
& {\left.[\rho+1(t))+\varepsilon \sigma\left(t, \Theta^{k}(t)\right)+\sigma_{0}(t)\right] d W(t) } \\
& d Y^{k+1}(t)= {\left[\rho g\left(t, \Theta^{k+1}(t)\right)+\varepsilon g\left(t, \Theta^{k}(t)\right)+g_{0}(t)\right] d t+Z^{k+1}(t) d W(t) } \\
& X^{k+1}(0)=\rho \gamma\left(X^{k+1}(T), Y^{k+1}(0)\right)+\varepsilon \gamma\left(X^{k}(T), Y^{k}(0)\right)+\gamma_{0} \\
& Y^{k+1}(T)=\rho h\left(X^{k+1}(T), Y^{k+1}(0)\right)+\varepsilon h\left(X^{k}(T), Y^{k}(0)\right)+h_{0}
\end{aligned}\right.
$$


By our assumption, inductively, for each $k \geq 0$, as long as $\Theta^{k}(\cdot) \in \mathcal{M}^{p}[0, T]$, the above FBSDE admits a unique adapted solution $\Theta^{k+1}(\cdot) \in \mathcal{M}^{p}[0, T]$. Furthermore, $(4.11)$

$$
\begin{aligned}
& \| \Theta^{k+1}(\cdot) \|_{\mathcal{M}^{p}[0, T]}^{p} \equiv \mathbb{E}\left\{\sup _{t \in[0, T]}\left|X^{k+1}(t)\right|^{p}+\sup _{t \in[0, T]}\left|Y^{k+1}(t)\right|^{p}+\left(\int_{0}^{T}\left|Z^{k+1}(t)\right|^{2} d t\right)^{\frac{p}{2}}\right\} \\
& \leq K \mathbb{E}\{(\mid\left.\left.\gamma(0,0)|+| \varepsilon \gamma\left(X^{k}(T), Y^{k}(0)\right)+\gamma_{0} \mid\right)^{p}+\left(|h(0,0)|+\left|\varepsilon h\left(X^{k}(T), Y^{k}(0)\right)+h_{0}\right|\right)^{p}\right\} \\
&+ {\left[\int_{0}^{T}\left(|b(t, 0)|+\left|\varepsilon b\left(t, \Theta^{k}(t)\right)+b_{0}(t)\right|\right)^{2} d t\right]^{\frac{p}{2}} } \\
&+ {\left[\int_{0}^{T}\left(|\sigma(t, 0)|+\left|\varepsilon \sigma\left(t, \Theta^{k}(t)\right)+\sigma_{0}(t)\right|\right)^{2} d t\right]^{\frac{p}{2}} } \\
&+ {\left[\int_{0}^{T}\left(|g(t, 0)|+\left|\varepsilon g\left(t, \Theta^{k}(t)\right)+g_{0}(t)\right|\right)^{2} d t\right]^{\frac{p}{2}} } \\
& \leq K \mathbb{E}\{[\mid\left.\gamma(0,0)|+| \gamma_{0} \mid+\varepsilon\left(\left|X^{k}(T)\right|+\left|Y^{k}(0)\right|\right)\right]^{p}+\left[|h(0,0)|+\left|h_{0}\right|+\varepsilon\left(\left|X^{k}(T)\right|+\left|Y^{k}(0)\right|\right)\right]^{p} \\
&+ {\left[\int_{0}^{T}\left(|b(t, 0)|+\left|b_{0}(t)\right|+\varepsilon\left|\Theta^{k}(t)\right|\right)^{2} d t\right]^{\frac{p}{2}} } \\
&+ {\left[\int_{0}^{T}\left(|g(t, 0)|+\left|g_{0}(t)\right|+\varepsilon\left|\Theta^{k}(t)\right|\right)^{2} d t\right]^{\frac{p}{2}} } \\
&+ {\left.\left[\int_{0}^{T}\left(|\sigma(t, 0)|+\left|\sigma_{0}(t)\right|+\varepsilon\left|\Theta^{k}(t)\right|\right)^{2} d t\right]^{\frac{p}{2}}\right\} } \\
& \leq K \varepsilon^{p}\left\|\Theta^{k}(\cdot)\right\|_{\mathcal{M}^{p}[0, T]}^{p}+K \mathbb{E}\left\{\left[|\gamma(0,0)|+\left|\gamma_{0}\right|\right]^{p}+\left[|h(0,0)|+\left|h_{0}\right|\right]^{p}\right. \\
&+\left[\int_{0}^{T}\left(|b(t, 0)|+\left|b_{0}(t)\right|\right)^{2} d t\right]^{\frac{p}{2}}+\left[\int_{0}^{T}\left(|g(t, 0)|+\left|g_{0}(t)\right|\right)^{2} d t\right]^{\frac{p}{2}} \\
&\left.+\left[\int_{0}^{T}\left(|\sigma(t, 0)|+\left|\sigma_{0}(t)\right|\right)^{2} d t\right]^{\frac{p}{2}}\right\} .
\end{aligned}
$$

Now, since $(b, \sigma, g, \gamma, h)$ satisfies (3.15) and (3.18) and admits a type (I) or type (II) Lyapunov operator $P(\cdot)$, by Proposition 3.4 , we obtain

$$
\begin{aligned}
& \left\|\Theta^{k+1}(\cdot)-\Theta^{k}(\cdot)\right\|_{\mathcal{M}^{2}[0, T]}^{2} \equiv \mathbb{E}\left\{\sup _{t \in[0, T]}\left|X^{k+1}(t)-X^{k}(t)\right|^{2}\right. \\
& \left.\quad+\sup _{t \in[0, T]}\left|Y^{k+1}(t)-Y^{k}(t)\right|^{2}+\int_{0}^{T}\left|Z^{k+1}(t)-Z^{k}(t)\right|^{2} d t\right\} \\
& \leq \varepsilon^{2} K \mathbb{E}\left\{\left|\gamma\left(X^{k}(T), Y^{k}(0)\right)-\gamma\left(X^{k-1}(T), Y^{k-1}(0)\right)\right|^{2}\right. \\
& +\left|h\left(X^{k}(T), Y^{k}(0)\right)-h\left(X^{k-1}(T), Y^{k-1}(0)\right)\right|^{2} \\
& \quad+\int_{0}^{T}\left[\left|b\left(t, \Theta^{k}(t)\right)-b\left(t, \Theta^{k-1}(t)\right)\right|^{2}+\mid \sigma\left(t, \Theta^{k}(t)\right)-\sigma\left(t,\left.\Theta^{k-1}(t)\right|^{2}\right.\right. \\
& \left.\quad+\mid g\left(t, \Theta^{k}(t)\right)-g\left(t,\left.\Theta^{k-1}(t)\right|^{2}\right] d t\right\} \\
& \leq \varepsilon^{2} K_{0}\left\|\Theta^{k}(\cdot)-\Theta^{k-1}(\cdot)\right\|_{\mathcal{M}^{2}[0, T]}^{2} .
\end{aligned}
$$

Here, $K_{0}>0$ is an absolute constant (independent of $k \geq 1$ ). Thus, taking $\varepsilon>0$ small enough so that $\varepsilon K_{0}^{\frac{1}{2}} \leq \frac{1}{2}$, we obtain

$$
\lim _{k \rightarrow \infty}\left\|\Theta^{k}(\cdot)-\Theta_{\rho+\varepsilon}(\cdot)\right\|_{\mathcal{M}^{2}[0, T]}=0,
$$


with

$$
\Theta_{\rho+\varepsilon}(\cdot)=\sum_{k=1}^{\infty}\left[\Theta^{k}(\cdot)-\Theta^{k-1}(\cdot)\right]
$$

which is the unique adapted solution of FBSDE (4.9). Furthermore, letting $k \rightarrow \infty$ in (4.11) and making use of Lemma 4.2, we obtain

$$
\begin{aligned}
& \left\|\Theta_{\rho+\varepsilon}(\cdot)\right\|_{\mathcal{M}^{p}[0, T]}^{p} \\
& \equiv \mathbb{E}\left\{\sup _{t \in[0, T]}\left|X_{\rho+\varepsilon}(t)\right|^{p}+\sup _{t \in[0, T]}\left|Y_{\rho+\varepsilon}(t)\right|^{p}+\left(\int_{0}^{T}\left|Z_{\rho+\varepsilon}(t)\right|^{2} d t\right)^{\frac{p}{2}}\right\} \\
& \leq K \varepsilon^{p}\left\|\Theta_{\rho+\varepsilon}(\cdot)\right\|_{\mathcal{M}^{p}[0, T]}^{p}+K \mathbb{E}\left\{\left[|\gamma(0,0)|+\left|\gamma_{0}\right|\right]^{p}+\left[|h(0,0)|+\left|h_{0}\right|\right]^{p}\right. \\
& +\left[\int_{0}^{T}\left(|b(t, 0)|+\left|b_{0}(t)\right|\right)^{2} d t\right]^{\frac{p}{2}}+\left[\int_{0}^{T}\left(|g(t, 0)|+\left|g_{0}(t)\right|\right)^{2} d t\right]^{\frac{p}{2}} \\
& \left.\quad+\left[\int_{0}^{T}\left(|\sigma(t, 0)|+\left|\sigma_{0}(t)\right|\right)^{2} d t\right]^{\frac{p}{2}}\right\} .
\end{aligned}
$$

Note that the constant $K$ in front of $\varepsilon^{p}$ above is universal. Then choose an $\varepsilon>0$ satisfying $K \varepsilon^{p} \leq \frac{1}{2}$ so that the first term on the right hand side can be absorbed into the left hand side, leading to the following:

$$
\begin{aligned}
& \left\|\Theta_{\rho+\varepsilon}(\cdot)\right\|_{\mathcal{M}^{p}[0, T]}^{p} \\
& \leq K \mathbb{E}\left\{\left[|\gamma(0,0)|+\left|\gamma_{0}\right|\right]^{p}+\left[|h(0,0)|+\left|h_{0}\right|\right]^{p}+\left[\int_{0}^{T}\left(|b(t, 0)|+\left|b_{0}(t)\right|\right)^{2} d t\right]^{\frac{p}{2}}\right. \\
& \left.\quad+\left[\int_{0}^{T}\left(|g(t, 0)|+\left|g_{0}(t)\right|\right)^{2} d t\right]^{\frac{p}{2}}+\left[\int_{0}^{T}\left(|\sigma(t, 0)|+\left|\sigma_{0}(t)\right|\right)^{2} d t\right]^{\frac{p}{2}}\right\} .
\end{aligned}
$$

Continuing the above procedure, we obtain the solvability of the following coupled FBSDE:

$$
\left\{\begin{array}{l}
d X(t)=\left[b(t, \Theta(t))+b_{0}(t)\right] d t+\left[\sigma(t, \Theta(t))+\sigma_{0}(t)\right] d W(t), \\
d Y(t)=\left[g(t, \Theta(t))+g_{0}(t)\right] d t+Z(t) d W(t), \\
X(0)=\gamma(X(T), Y(0))+\gamma_{0}, \quad Y(T)=h(X(T), Y(0))+h_{0}
\end{array}\right.
$$

with the solution $\Theta(\cdot)$ satisfying

$$
\begin{aligned}
& \|\Theta(\cdot)\|_{\mathcal{M}^{p}[0, T]}^{p} \\
& \leq K \mathbb{E}\left\{\left[|\gamma(0,0)|+\left|\gamma_{0}\right|\right]^{p}+\left[|h(0,0)|+\left|h_{0}\right|\right]^{p}+\left[\int_{0}^{T}\left(|b(t, 0)|+\left|b_{0}(t)\right|\right)^{2} d t\right]^{\frac{p}{2}}\right. \\
& \left.\quad+\left[\int_{0}^{T}\left(|g(t, 0)|+\left|g_{0}(t)\right|\right)^{2} d t\right]^{\frac{p}{2}}+\left[\int_{0}^{T}\left(|\sigma(t, 0)|+\left|\sigma_{0}(t)\right|\right)^{2} d t\right]^{\frac{p}{2}}\right\} .
\end{aligned}
$$


Thus, in particular, by taking $\left(b_{0}, \sigma_{0}, g_{0}, \gamma_{0}, h_{0}\right)=0$, we obtain the solvability of FBSDE (1.1) with the estimate

$$
\begin{gathered}
\|\Theta(\cdot)\|_{\mathcal{M}^{p}[0, T]}^{p} \equiv \mathbb{E}\left\{\sup _{t \in[0, T]}|X(t)|^{p}+\sup _{t \in[0, T]}|Y(t)|^{p}+\left(\int_{0}^{T}|Z(t)|^{2} d t\right)^{\frac{p}{2}}\right\} \\
\leq K \mathbb{E}\left\{|\gamma(0,0)|^{p}+|h(0,0)|^{p}+\left(\int_{0}^{T}|b(t, 0)|^{2} d t\right)^{\frac{p}{2}}+\left(\int_{0}^{T}|g(t, 0)|^{2} d t\right)^{\frac{p}{2}}\right. \\
\left.+\left(\int_{0}^{T}|\sigma(t, 0)|^{2} d t\right)^{\frac{p}{2}}\right\} .
\end{gathered}
$$

Now, let $\bar{\Theta}(\cdot)$ be an adapted solution to (1.1) corresponding to $(\bar{b}, \bar{\sigma}, \bar{g}, \bar{\gamma}, \bar{h}) \in \widetilde{\mathcal{G}}^{p}$. Then, crucially, $\bar{\Theta}(\cdot)-\Theta(\cdot)$ satisfies a linear FBSDE with the generator admitting a type (I) or type (II) Lyapunov operator $P(\cdot)$ the same as that for the generator $(b, \sigma, g, \gamma, h)$, and the corresponding conditions (3.15) and (3.18) hold. Hence, applying (4.19), we obtain the following stability estimate:

$$
\begin{aligned}
\| \bar{\Theta}(\cdot)- & \Theta(\cdot) \|_{\mathcal{M}^{p}[0, T]}^{p} \equiv \mathbb{E}\left\{\sup _{t \in[0, T]}|\bar{X}(t)-X(t)|^{p}+\sup _{t \in[0, T]}|\bar{Y}(t)-Y(t)|^{p}\right. \\
& \left.+\left(\int_{0}^{T}|\bar{Z}(t)-Z(t)|^{2} d t\right)^{\frac{p}{2}}\right\} \\
\leq K \mathbb{E}\{\mid & |\bar{\gamma}(\bar{X}(T), \bar{Y}(0))-\gamma(\bar{X}(T), \bar{Y}(0))|^{p} \\
& +|\bar{h}(\bar{X}(T), \bar{Y}(0))-h(\bar{X}(T), \bar{Y}(0))|^{p} \\
& +\left(\int_{0}^{T}|\bar{b}(t, \bar{\Theta}(t))-b(t, \bar{\Theta}(t))|^{2} d t\right)^{\frac{p}{2}} \\
& +\left(\int_{0}^{T}|\bar{g}(t, \bar{\Theta}(t))-g(t, \bar{\Theta}(t))|^{2} d t\right)^{\frac{p}{2}} \\
& \left.+\left(\int_{0}^{T}|\bar{\sigma}(t, \bar{\Theta}(t))-\sigma(t, \bar{\Theta}(t))|^{2} d t\right)^{\frac{p}{2}}\right\} .
\end{aligned}
$$

Note that (4.19) and (4.20) are not yet the same as (4.1) and (4.2). We need to refine those. To this end, we let $\bar{\Theta}(\cdot) \equiv(\bar{X}(\cdot), \bar{Y}(\cdot), \bar{Z}(\cdot)) \in \mathcal{M}^{p}[0, T]$ be the adapted solution to the following FBSDE:

$$
\left\{\begin{array}{l}
d \bar{X}(t)=b(t, 0) d t+\sigma(t, 0) d W(t), \\
d \bar{Y}(t)=g(t, 0) d t+\bar{Z}(t) d W(t), \\
\bar{X}(0)=\gamma(0,0), \quad \bar{Y}(T)=h(0,0) .
\end{array}\right.
$$

Then it is standard that

$$
\begin{aligned}
& \|\bar{\Theta}(\cdot)\|_{\mathcal{M}^{p}[0, T]}^{p} \equiv \mathbb{E}\left\{\sup _{t \in[0, T]}|\bar{X}(t)|^{p}+\sup _{t \in[0, T]}|\bar{Y}(t)|^{p}+\left(\int_{0}^{T}|\bar{Z}(t)|^{2} d t\right)^{\frac{p}{2}}\right\} \\
& \leq K \mathbb{E}\left\{|\gamma(0,0)|^{p}+|h(0,0)|^{p}+\left(\int_{0}^{T}|b(t, 0)| d t\right)^{p}+\left(\int_{0}^{T}|g(t, 0)| d t\right)^{p}\right. \\
& \left.\quad+\left(\int_{0}^{T}|\sigma(t, 0)|^{2} d t\right)^{\frac{p}{2}}\right\} .
\end{aligned}
$$


Consequently, the above, together with (4.20), gives

$$
\begin{aligned}
& \|\Theta(\cdot)\|_{\mathcal{M}^{p}[0, T]}^{p} \leq 2^{p-1}\|\bar{\Theta}(\cdot)-\Theta(\cdot)\|_{\mathcal{M}^{p}[0, T]}^{p}+2^{p-1}\|\bar{\Theta}(\cdot)\|_{\mathcal{M}^{p}[0, T]}^{p} \\
& \leq K \mathbb{E}\left\{|\gamma(0,0)-\gamma(\bar{X}(T), \bar{Y}(0))|^{p}+|h(0,0)-h(\bar{X}(T), \bar{Y}(0))|^{p}\right. \\
& \quad+\left(\int_{0}^{T}|b(t, 0)-b(t, \bar{\Theta}(t))|^{2} d t\right)^{\frac{p}{2}}+\left(\int_{0}^{T}|g(t, 0)-g(t, \bar{\Theta}(t))|^{2} d t\right)^{\frac{p}{2}} \\
& \left.\quad+\left(\int_{0}^{T}|\sigma(t, 0)-\sigma(t, \bar{\Theta}(t))|^{2} d t\right)^{\frac{p}{2}}+\|\bar{\Theta}(\cdot)\|_{\mathcal{M}^{p}[0, T]}^{p}\right\} \\
& \leq K\|\bar{\Theta}(\cdot)\|_{\mathcal{M}^{p}[0, T]}^{p} \leq K \mathbb{E}\left\{|\gamma(0,0)|^{p}+|h(0,0)|^{p}\right. \\
& \left.\quad+\left(\int_{0}^{T}|b(t, 0)| d t\right)^{p}+\left(\int_{0}^{T}|g(t, 0)| d t\right)^{p}+\left(\int_{0}^{T}|\sigma(t, 0)|^{2} d t\right)^{\frac{p}{2}}\right\} .
\end{aligned}
$$

Thus, (4.1) holds, and then (4.2) holds. This proves the theorem for any generator $(b, \sigma, g, \gamma, h) \in \widetilde{\mathcal{G}}^{p}$. Finally, by a usual approximation argument, we can show that the theorem also holds for any generator $(b, \sigma, g, \gamma, h) \in \mathcal{G}^{p}$.

Comparing the above proof with that in [28, we can see the following major differences. (i) Lemma 4.2 is used to obtain the existence of the $L^{p}$-adapted solution, together with the estimates (4.19)-(4.20), for generators in $\widetilde{\mathcal{G}}^{p}$. (ii) Due to the definition of the Lyapunov operator, we have that the linear FBSDE satisfied by $\bar{\Theta}(\cdot)-\Theta(\cdot)$ admits the same Lyapunov operator $P(\cdot)$ as that of $(b, \sigma, g, \gamma, h)$. Thus, no additional condition is needed to obtain the stability estimate (4.20). (iii) We used a "boot-strap" idea to refine estimates (4.19)-(4.20) to obtain estimates (4.1)(4.2), which will be very useful in the study of optimal control for FBSDEs.

\section{EXistence of Lyapunov operators - GENERAl CONSIDERATIONS}

From the previous section, we have seen that the existence of a Lyapunov operator (together with conditions (3.15) and (3.18) ) guarantees the well-posedness of FBSDE (1.1). In this and the following sections, we will obtain the existence of special form Lyapunov operators under various (additional) conditions on the generator $(b, \sigma, g, \gamma, h) \in \mathcal{G}^{2}$. To begin with, let us introduce the following notation:

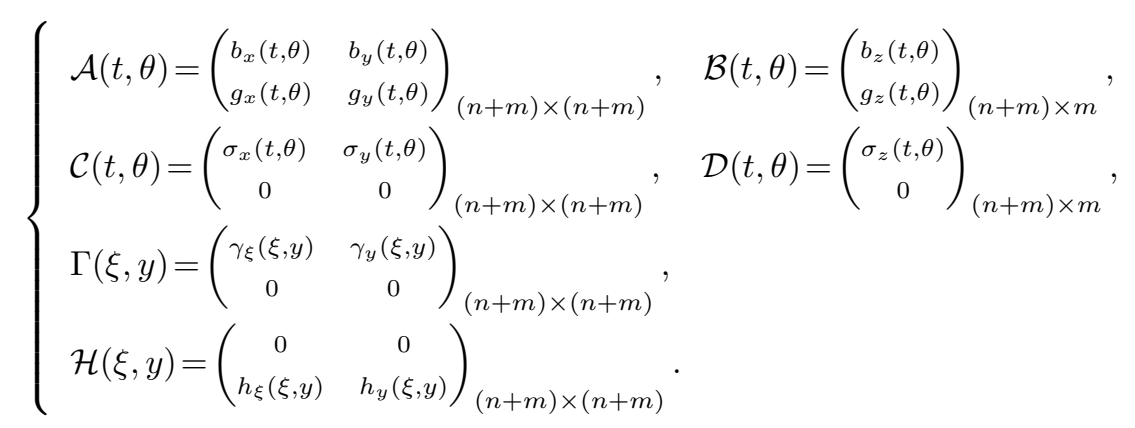


Then (suppressing the arguments $(t, \theta)$ and $(\xi, y))$

$$
\begin{aligned}
& \mathbf{F}(P(\cdot), 1 ; t, \theta) \\
& =\left(\begin{array}{cccc}
\dot{P}+P \mathcal{A}+\mathcal{A}^{T} P+\mathcal{C}^{T} P \mathcal{C} & P \mathcal{B}+\mathcal{C}^{T} P\left(L_{m}+\mathcal{D}\right) & P & \mathcal{C}^{T} P L_{n} \\
\mathcal{B}^{T} P+\left(L_{m}+\mathcal{D}\right)^{T} P \mathcal{C} & \left(L_{m}+\mathcal{D}\right)^{T} P\left(L_{m}+\mathcal{D}\right) & 0 & \left(L_{m}+\mathcal{D}\right)^{T} P L_{n} \\
P & 0 & 0 I_{n+m} & 0 \\
L_{n}^{T} P \mathcal{C} & L_{n}^{T} P\left(L_{m}+\mathcal{D}\right) & 0 & L_{n}^{T} P L_{n}
\end{array}\right) \\
& \equiv\left(\begin{array}{cc}
I_{n+m} \\
F(P(\cdot) ; t, \theta) & \left(\begin{array}{c}
\left(L_{m}+\mathcal{D}\right)^{T} \\
\equiv
\end{array}\right) P\left(I_{n+m}, L_{n}\right) \\
\left(\begin{array}{c}
I_{n+m} \\
L_{n}^{T}
\end{array}\right) P\left(I_{n+m}, L_{m}+\mathcal{D}\right) & \left(\begin{array}{c}
0 \\
L_{n}^{T}
\end{array}\right) P\left(0, L_{n}\right)
\end{array}\right)
\end{aligned}
$$

and

$$
\begin{aligned}
& \mathbf{G}(P(\cdot), 1 ; \xi, y)=\left(\begin{array}{c}
J_{n}+\mathcal{H}^{*} \\
J_{m}
\end{array}\right) P(T)\left(J_{n}+\mathcal{H}, J_{m}\right)-\left(\begin{array}{c}
J_{m}+\Gamma^{*} \\
J_{n}
\end{array}\right) P(0)\left(J_{m}+\Gamma, J_{n}\right) \\
& =\left(\begin{array}{cc}
\left(J_{n}+\mathcal{H}^{*}\right) P(T)\left(J_{n}+\mathcal{H}\right)-\left(J_{m}+\Gamma^{*}\right) P(0)\left(J_{m}+\Gamma\right) & \left(J_{n}+\mathcal{H}^{*}\right) P(T) J_{m}-\left(J_{m}+\Gamma^{*}\right) P(0) J_{n} \\
J_{m} P(T)\left(J_{n}+\mathcal{H}\right)-J_{n} P(0)\left(J_{m}+\Gamma\right) & J_{m} P(T) J_{m}-J_{n} P(0) J_{n}
\end{array}\right) \\
& \equiv\left(\begin{array}{cc}
G(P(\cdot) ; \xi, y) & \left(J_{n}+\mathcal{H}^{*}\right) P(T) J_{m}-\left(J_{m}+\Gamma^{*}\right) P(0) J_{n} \\
J_{m} P(T)\left(J_{n}+\mathcal{H}\right)-J_{n} P(0)\left(J_{m}+\Gamma\right) & J_{m} P(T) J_{m}-J_{n} P(0) J_{n}
\end{array}\right),
\end{aligned}
$$

with

$$
F(P(\cdot) ; t, \theta)=\left(\begin{array}{cc}
\dot{P}+P \mathcal{A}+\mathcal{A}^{T} P+\mathcal{C}^{T} P \mathcal{C} & P \mathcal{B}+\mathcal{C}^{T} P\left(L_{m}+\mathcal{D}\right) \\
\mathcal{B}^{T} P+\left(L_{m}+\mathcal{D}\right)^{T} P \mathcal{C} & \left(L_{m}+\mathcal{D}\right)^{T} P\left(L_{m}+\mathcal{D}\right)
\end{array}\right)
$$

and

$$
G(P(\cdot) ; \xi, y)=\left(J_{n}+\mathcal{H}^{*}\right) P(T)\left(J_{n}+\mathcal{H}\right)-\left(J_{m}+\Gamma^{*}\right) P(0)\left(J_{m}+\Gamma\right) .
$$

It is seen that for any given $(P(\cdot) ; t, \theta)$ (and $\omega \in \Omega$ ), $F(P(\cdot) ; t, \theta) \in \mathcal{S}^{n+2 m}$, and for any given $(P(\cdot), \xi, y)$ (and $\omega \in \Omega$ ), $G(P(\cdot) ; \xi, y): \mathcal{X}_{n}^{2} \times \mathbb{R}^{m} \rightarrow \mathcal{X}_{n}^{2} \times \mathbb{R}^{m}$ is a symmetric bounded linear operator. Thus, $F(P(\cdot) ; t, \theta)$ is an $\mathcal{S}^{n+2 m}$-valued random variable and $G(P(\cdot) ; \xi, y)$ is a linear operator between two Hilbert spaces.

We denote

$$
\left\{\begin{array}{l}
\Phi_{g}(t, \theta)=\left(\begin{array}{cc}
\left\{g_{x}^{T} g_{x}\right\}_{\infty} & 0 \\
0 & 0 I_{2 m}
\end{array}\right)_{(n+2 m) \times(n+2 m)} \\
\Phi_{b \sigma}(t, \theta)=\left(\begin{array}{cc}
0 I_{n} & 0 \\
0 & \left\{\left(\begin{array}{c}
b_{y}^{T} \\
b_{z}^{T}
\end{array}\right)\left(b_{y}, b_{z}\right)+\left(\begin{array}{c}
\sigma_{y}^{T} \\
\sigma_{z}^{T}
\end{array}\right)\left(\sigma_{y}, \sigma_{z}\right)\right\}_{\infty}
\end{array}\right)_{(n+2 m) \times(n+2 m)} .
\end{array}\right.
$$

Then $P(\cdot)$ is respectively a Lyapunov operator of types (I), (II), and (III) if (3.24) holds and we respectively have

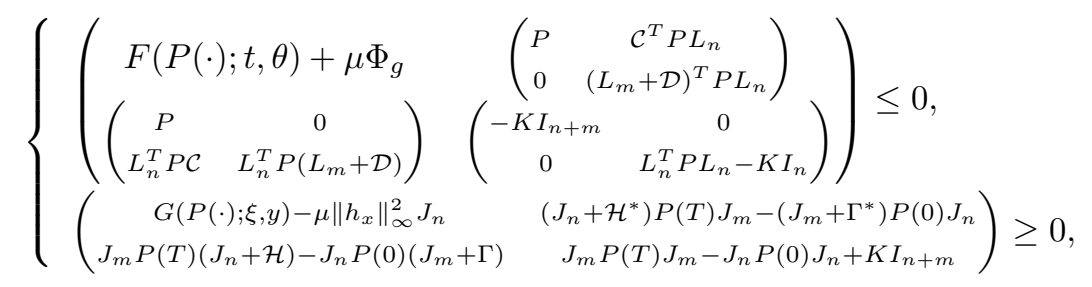




$$
\left\{\begin{array}{c}
\left(\begin{array}{c}
F(P(\cdot) ; t, \theta)+\mu \Phi_{b \sigma} \\
\left(\begin{array}{cc}
P & \left(\begin{array}{cc}
P & \mathcal{C}^{T} P L_{n} \\
0 & \left(L_{m}+\mathcal{D}\right)^{T} P L_{n}
\end{array}\right) \\
L_{n}^{T} P \mathcal{C} & L_{n}^{T} P\left(L_{m}+\mathcal{D}\right)
\end{array}\right)
\end{array} \quad\left(\begin{array}{cc}
-K I_{n+m} & 0 \\
0 & L_{n}^{T} P L_{n}-K I_{n}
\end{array}\right)\right) \leq 0 \\
\left(\begin{array}{cc}
G(P(\cdot) ; \xi, y)-\mu\left\|\gamma_{y}\right\|_{\infty}^{2} J_{m} & \left(J_{n}+\mathcal{H}^{*}\right) P(T) J_{m}-\left(J_{m}+\Gamma^{*}\right) P(0) J_{n} \\
J_{m} P(T)\left(J_{n}+\mathcal{H}\right)-J_{n} P(0)\left(J_{m}+\Gamma\right) & J_{m} P(T) J_{m}-J_{n} P(0) J_{n}+K I_{n+m}
\end{array}\right) \geq 0
\end{array}\right.
$$

and

$$
\left\{\begin{array}{c}
\left(\begin{array}{cc}
F(P(\cdot) ; t, \theta)+\mu I_{n+2 m} & \left(\begin{array}{cc}
P & \mathcal{C}^{T} P L_{n} \\
0 & \left(L_{m}+\mathcal{D}\right)^{T} P L_{n}
\end{array}\right) \\
\left(\begin{array}{cc}
P & 0 \\
L_{n}^{T} P \mathcal{C} & L_{n}^{T} P\left(L_{m}+\mathcal{D}\right)
\end{array}\right) & \left.\left(\begin{array}{cc}
-K I_{n+m} & 0 \\
0 & L_{n}^{T} P L_{n}-K I_{n}
\end{array}\right)\right) \leq 0 \\
G(P(\cdot) ; \xi, y)-\mu I_{n+m} & \left(J_{n}+\mathcal{H}^{*}\right) P(T) J_{m}-\left(J_{m}+\Gamma^{*}\right) P(0) J_{n} \\
J_{m} P(T)\left(J_{n}+\mathcal{H}\right)-J_{n} P(0)\left(J_{m}+\Gamma\right) & J_{m} P(T) J_{m}-J_{n} P(0) J_{n}+K I_{n+m}
\end{array}\right) \geq 0 .
\end{array}\right.
$$

We now present the following useful proposition whose proof is pretty straightforward.

Proposition 5.1. Let $(b, \sigma, g, \gamma, h) \in \mathcal{G}^{2}$. Suppose $P:[0, T] \rightarrow \mathcal{S}^{n+m}$ satisfies (3.24). Then $P(\cdot)$ is a type (III) Lyapunov operator of the generator $(b, \sigma, g, \gamma, h)$ if and only if

$$
F(P(\cdot) ; \cdot, \cdot) \ll 0, \quad G(P(\cdot) ; \cdot, \cdot) \gg 0 .
$$

Since a type (III) Lyapunov operator is automatically a type (I) and type (II) Lyapunov operator, the above gives a set of sufficient conditions for the existence of the latter. It is not hard to see that (5.10) is equivalent to the following:

$$
\left\{\begin{array}{l}
\left(L_{m}+\mathcal{D}\right)^{T} P\left(L_{m}+\mathcal{D}\right) \ll 0 \\
\dot{P}+P \mathcal{A}+\mathcal{A}^{T} P+\mathcal{C}^{T} P \mathcal{C} \\
-\left[P \mathcal{B}+\mathcal{C}^{T} P\left(L_{m}+\mathcal{D}\right)\right]\left[\left(L_{m}+\mathcal{D}\right)^{T} P\left(L_{m}+\mathcal{D}\right)\right]^{-1}\left[\mathcal{B}^{T} P+\left(L_{m}+\mathcal{D}\right)^{T} P \mathcal{C}\right] \ll 0 \\
\left(J_{n}+\mathcal{H}^{*}\right) P(T)\left(J_{n}+\mathcal{H}\right)-\left(J_{m}+\Gamma^{*}\right) P(0)\left(J_{m}+\Gamma\right) \gg 0
\end{array}\right.
$$

The above is referred to as Riccati differential inequalities with constraints. The first inequality is an interior pointwise constraint for $P(\cdot)$; the last inequality is a boundary constraint for $P(\cdot)$.

The following proposition gives some sufficient conditions under which a special form of type (III) Lyapunov operator $P(\cdot)$ exists.

Proposition 5.2. Let $(b, \sigma, g, \gamma, h) \in \mathcal{G}^{2}$. Suppose for some matrix $\Psi \in \mathbb{R}^{m \times n}$ and constants $\nu_{1}, \nu_{2}, \kappa_{1}, \kappa_{2}>0$,

$$
\begin{gathered}
\left(\begin{array}{cc}
\nu_{1}\left(I-e^{\kappa_{1} T} \gamma_{\xi}^{*} \gamma_{\xi}\right)-\nu_{2} e^{\kappa_{2} T} h_{\xi}^{*} h_{\xi} & -\nu_{1} e^{\kappa_{1} T} \gamma_{\xi}^{*} \gamma_{y}-\nu_{2} e^{\kappa_{2} T} h_{\xi}^{*} h_{y} \\
-\nu_{1} e^{\kappa_{1} T} \gamma_{y}^{T} \gamma_{\xi}-\nu_{2} e^{\kappa_{2} T} h_{y}^{*} h_{\xi} & \nu_{2}\left(I-e^{\kappa_{2} T} h_{y}^{*} h_{y}\right)-\nu_{1} e^{\kappa_{1} T} \gamma_{y}^{T} \gamma_{y}
\end{array}\right) \\
\quad+\left(\begin{array}{cc}
\Psi^{T} h_{\xi}+h_{\xi}^{*} \Psi & \Psi^{T} h_{y}-\gamma_{\xi}^{*} \Psi^{T} \\
-\Psi \gamma_{\xi}+h_{y}^{*} \Psi & -\Psi \gamma_{y}-\gamma_{y}^{T} \Psi^{T}
\end{array}\right) \gg 0
\end{gathered}
$$




$$
\begin{aligned}
& \left(\begin{array}{ccc}
\nu_{1} e^{\kappa_{1}(T-t)}\left(b_{x}+b_{x}^{T}+\sigma_{x}^{T} \sigma_{x}-\kappa_{1} I\right) & \nu_{1} e^{\kappa_{1}(T-t)}\left(b_{y}+\sigma_{x}^{T} \sigma_{y}\right)-\nu_{2} e^{\kappa_{2} t} g_{x}^{T} & \nu_{1} e^{\kappa_{1}(T-t)}\left(b_{z}+\sigma_{x}^{T} \sigma_{z}\right) \\
\nu_{1} e^{\kappa_{1}(T-t)}\left(b_{y}^{T}+\sigma_{y}^{T} \sigma_{x}\right)-\nu_{2} e^{\kappa_{2} t} g_{x} & \nu_{1} e^{\kappa_{1}(T-t)} \sigma_{y}^{T} \sigma_{y}-\nu_{2} e^{\kappa_{2} t}\left(\kappa_{2} I+g_{y}+g_{y}^{T}\right) & \nu_{1} e^{\kappa_{1}(T-t)} \sigma_{y}^{T} \sigma_{z}-\nu_{2} e^{\kappa_{2} t} g_{z} \\
\nu_{1} e^{\kappa_{1}(T-t)}\left(b_{z}^{T}+\sigma_{z}^{T} \sigma_{x}\right) & \nu_{1} e^{\kappa_{1}(T-t)} \sigma_{z}^{T} \sigma_{y}-\nu_{2} e^{\kappa_{2} t} g_{z}^{T} & \nu_{1} e^{\kappa_{1}(T-t)} \sigma_{z}^{T} \sigma_{z}-\nu_{2} e^{\kappa_{2} t} I
\end{array}\right) \\
& \quad+\left(\begin{array}{ccc}
\Psi^{T} g_{x}+g_{x}^{T} \Psi & \Psi^{T} g_{y}+b_{x}^{T} \Psi^{T} & \Psi^{T} g_{z}+\sigma_{x}^{T} \Psi^{T} \\
\Psi b_{x}+g_{y}^{T} \Psi & \Psi b_{y}+b_{y}^{T} \Psi^{T} & \Psi b_{z}+\sigma_{y}^{T} \Psi^{T} \\
\Psi \sigma_{x}+g_{z}^{T} \Psi & \Psi \sigma_{y}+b_{z}^{T} \Psi^{T} & \Psi \sigma_{z}+\sigma_{z}^{T} \Psi^{T}
\end{array}\right) \ll 0 .
\end{aligned}
$$

Then the following is a type (III) Lyapunov operator of the generator $(b, \sigma, g, \gamma, h)$ :

$$
P(t)=\left(\begin{array}{cc}
p_{1}(t) I_{n} & \Psi^{T} \\
\Psi & p_{2}(t) I_{m}
\end{array}\right) \equiv\left(\begin{array}{cc}
\nu_{1} e^{\kappa_{1}(T-t)} I & \Psi^{T} \\
\Psi & -\nu_{2} e^{\kappa_{2} t} I
\end{array}\right), \quad t \in[0, T] .
$$

Proof. It is clear from (5.14) that

$$
p_{1}(t) \equiv \nu_{1} e^{\kappa_{1}(T-t)} \geq \nu_{1}>0, \quad p_{2}(t) \equiv-\nu_{2} e^{\kappa_{2} t} \leq-\nu_{2}<0, \quad t \in[0, T],
$$

and

$$
\dot{P}(t)=\left(\begin{array}{cc}
-\kappa_{1} \nu_{1} e^{\kappa_{1}(T-t)} I & 0 \\
0 & -\kappa_{2} \nu_{2} e^{\kappa_{2} t} I
\end{array}\right) \leq\left(\begin{array}{cc}
-\kappa_{1} \nu_{1} I & 0 \\
0 & -\kappa_{2} \nu_{2} I
\end{array}\right)<0, \quad t \in[0, T] .
$$

Thus, (3.24) holds. Next, the last inequality in (5.11) is equivalent to the following:

$$
\begin{aligned}
& \left(\begin{array}{ll}
I & h_{\xi}^{*} \\
0 & h_{y}^{*}
\end{array}\right) P(T)\left(\begin{array}{cc}
I & 0 \\
h_{\xi} & h_{y}
\end{array}\right)-\left(\begin{array}{cc}
\gamma_{\xi}^{*} & 0 \\
\gamma_{y}^{T} & I
\end{array}\right) P(0)\left(\begin{array}{cc}
\gamma_{\xi} & \gamma_{y} \\
0 & I
\end{array}\right) \\
& =\left(\begin{array}{ll}
I & h_{\xi}^{*} \\
0 & h_{y}^{*}
\end{array}\right)\left(\begin{array}{cc}
p_{1}(T) I & 0 \\
0 & p_{2}(T) I
\end{array}\right)\left(\begin{array}{cc}
I & 0 \\
h_{\xi} & h_{y}
\end{array}\right)-\left(\begin{array}{cc}
\gamma_{\xi}^{*} & 0 \\
\gamma_{y}^{T} & I
\end{array}\right)\left(\begin{array}{cc}
p_{1}(0) I & 0 \\
0 & p_{2}(0) I
\end{array}\right)\left(\begin{array}{cc}
\gamma_{\xi} & \gamma_{y} \\
0 & I
\end{array}\right) \\
& \quad+\left(\begin{array}{cc}
I & h_{\xi}^{*} \\
0 & h_{y}^{*}
\end{array}\right)\left(\begin{array}{cc}
0 & \Psi^{T} \\
\Psi & 0
\end{array}\right)\left(\begin{array}{cc}
I & 0 \\
h_{\xi} & h_{y}
\end{array}\right)-\left(\begin{array}{cc}
\gamma_{\xi}^{*} & 0 \\
\gamma_{y}^{T} & I
\end{array}\right)\left(\begin{array}{cc}
0 & \Psi^{T} \\
\Psi & 0
\end{array}\right)\left(\begin{array}{cc}
\gamma_{\xi} & \gamma_{y} \\
0 & I
\end{array}\right) \\
& =\left(\begin{array}{cc}
\nu_{1}\left(I-e^{\kappa_{1} T} \gamma_{\xi}^{*} \gamma_{\xi}\right)-\nu_{2} e^{\kappa_{2} T} h_{\xi}^{*} h_{\xi} & -\nu_{1} e^{\kappa_{1} T} \gamma_{\xi}^{*} \gamma_{y}-\nu_{2} e^{\kappa_{2} T} h_{\xi}^{*} h_{y} \\
-\nu_{1} e^{\kappa_{1} T} \gamma_{y}^{T} \gamma_{\xi}-\nu_{2} e^{\kappa_{2} T} h_{y}^{*} h_{\xi} & \nu_{2}\left(I-e^{\kappa_{2} T} h_{y}^{*} h_{y}\right)-\nu_{1} e^{\kappa_{1} T} \gamma_{y}^{T} \gamma_{y}
\end{array}\right) \\
& \quad+\left(\begin{array}{ll}
\Psi^{T} h_{\xi}+h_{\xi}^{*} \Psi & \Psi^{T} h_{y}-\gamma_{\xi}^{*} \Psi^{T} \\
-\Psi \gamma_{\xi}+h_{y}^{*} \Psi & -\Psi \gamma_{y}-\gamma_{y}^{T} \Psi^{T}
\end{array}\right) \gg 0
\end{aligned}
$$

which is nothing but (5.12). Now, we note that

$$
\begin{aligned}
\left(L_{m}+\mathcal{D}\right)^{T} P\left(L_{m}+\mathcal{D}\right) & =p_{2}(t) I+p_{1}(t) \sigma_{z}^{T} \sigma_{z}+\sigma_{z}^{T} \Psi^{T}+\Psi \sigma_{z} \\
& =-\nu_{2} e^{\kappa_{2} t} I+\nu_{1} e^{\kappa_{1}(T-t)} \sigma_{z}^{T} \sigma_{z}+\sigma_{z}^{T} \Psi^{T}+\Psi \sigma_{z}
\end{aligned}
$$

and

$$
\begin{gathered}
P \mathcal{B}+\mathcal{C}^{T} P\left(L_{m}+\mathcal{D}\right)=\left(\begin{array}{cc}
p_{1} I & \Psi^{T} \\
\Psi & p_{2} I
\end{array}\right)\left(\begin{array}{c}
b_{z} \\
g_{z}
\end{array}\right)+\left(\begin{array}{cc}
\sigma_{x}^{T} & 0 \\
\sigma_{y}^{T} & 0
\end{array}\right)\left(\begin{array}{cc}
p_{1} I & \Psi^{T} \\
\Psi & p_{2} I
\end{array}\right)\left(\begin{array}{c}
\sigma_{z} \\
I
\end{array}\right) \\
=\left(\begin{array}{c}
\nu_{1} e^{\kappa_{1}(T-t)}\left(b_{z}+\sigma_{x}^{T} \sigma_{z}\right) \\
\nu_{1} e^{\kappa_{1}(T-t)} \sigma_{y}^{T} \sigma_{z}-\nu_{2} e^{\kappa_{2} t} g_{z}
\end{array}\right)+\left(\begin{array}{c}
\Psi^{T} g_{z}+\sigma_{x}^{T} \Psi^{T} \\
\Psi b_{z}+\sigma_{y}^{T} \Psi^{T}
\end{array}\right) .
\end{gathered}
$$


On the other hand,

$$
\begin{aligned}
\dot{P}+ & P \mathcal{A}+\mathcal{A}^{T} P+\mathcal{C}^{T} P \mathcal{C} \\
= & \left(\begin{array}{cc}
\dot{p}_{1} I & 0 \\
0 & \dot{p}_{2} I
\end{array}\right)+\left(\begin{array}{cc}
p_{1} I & \Psi^{T} \\
\Psi & p_{2} I
\end{array}\right)\left(\begin{array}{cc}
b_{x} & b_{y} \\
g_{x} & g_{y}
\end{array}\right)+\left(\begin{array}{cc}
b_{x}^{T} & g_{x}^{T} \\
b_{y}^{T} & g_{y}^{T}
\end{array}\right)\left(\begin{array}{cc}
p_{1} I & \Psi^{T} \\
\Psi & p_{2} I
\end{array}\right) \\
& \quad+\left(\begin{array}{cc}
\sigma_{x}^{T} & 0 \\
\sigma_{y}^{T} & 0
\end{array}\right)\left(\begin{array}{cc}
p_{1} I & \Psi^{T} \\
\Psi & p_{2} I
\end{array}\right)\left(\begin{array}{cc}
\sigma_{x} & \sigma_{y} \\
0 & 0
\end{array}\right) \\
= & \left(\begin{array}{cc}
\dot{p}_{1} I+p_{1}\left(b_{x}+b_{x}^{T}+\sigma_{x}^{T} \sigma_{x}\right)+\Psi^{T} g_{x}+g_{x}^{T} \Psi & p_{1}\left(b_{y}+\sigma_{x}^{T} \sigma_{y}\right)+p_{2} g_{x}^{T}+b_{x}^{T} \Psi^{T}+\Psi^{T} g_{y} \\
p_{1}\left(b_{y}^{T}+\sigma_{y}^{T} \sigma_{x}\right)+p_{2} g_{x}+\Psi b_{x}+g_{y}^{T} \Psi & \dot{p}_{2} I+p_{2}\left(g_{y}+g_{y}^{T}\right)+p_{1} \sigma_{y}^{T} \sigma_{y}+\Psi b_{y}+b_{y}^{T} \Psi^{T}
\end{array}\right) \\
= & \left(\begin{array}{cc}
\nu_{1} e^{\kappa_{1}(T-t)}\left(-\kappa_{1} I+b_{x}+b_{x}^{T}+\sigma_{x}^{T} \sigma_{x}\right) & \nu_{1} e^{\kappa_{1}(T-t)}\left(b_{y}+\sigma_{x}^{T} \sigma_{y}\right)-\nu_{2} e^{\nu_{2} t} g_{x}^{T} \\
\nu_{1} e^{\kappa_{1}(T-t)}\left(b_{y}^{T}+\sigma_{y}^{T} \sigma_{x}\right)-\nu_{2} e^{\kappa_{2} t} g_{x} & \nu_{1} e^{\kappa_{1}(T-t)} \sigma_{y}^{T} \sigma_{y}-\nu_{2} e^{\kappa_{2} t}\left(\kappa_{2} I+g_{y}+g_{y}^{T}\right)
\end{array}\right) \\
& \quad+\left(\begin{array}{cc}
\Psi^{T} g_{x}+g_{x}^{T} \Psi & \Psi^{T} g_{y}+b_{x}^{T} \Psi^{T} \\
\Psi b_{x}+g_{y}^{T} \Psi & \Psi b_{y}+b_{y}^{T} \Psi^{T}
\end{array}\right) .
\end{aligned}
$$

Hence, the inequality in the second line of (5.11) is equivalent to (5.13). Then our conclusion follows.

\section{Existence of Lyapunov operators — SPECIAL CASES}

We note that the conditions given in Proposition 5.2 are still not easy to use. In this section, we would like to look at some specific cases, showing a way that one could use Proposition 5.2 to find generators for which a type (III) Lyapunov operator $P(\cdot)$ of the form (5.14) exists. For simplicity of presentation, we do not attempt to get into the most general situations.

6.1. Decoupled cases. Although the solvability of decoupled FBSDEs can be obtained without the existence of a Lyapunov operator, we would like to look at the corresponding type (III) Lyapunov operators for these FBSDEs to get some feeling.

There are two types of decoupled FBSDEs. The first type is as follows:

$$
\left\{\begin{array}{l}
d X(t)=b(t, X(t)) d t+\sigma(t, X(t)) d W(t), \\
d Y(t)=g(t, X(t), Y(t), Z(t)) d t+Z(t) d W(t), \\
X(0)=X_{0}, \quad Y(T)=h(X(T)),
\end{array}\right.
$$

where $X_{0} \in \mathbb{R}^{n}$. One can simply solve the forward equation first, then solve the backward equation. Thus, under $(\mathrm{H} 1)_{p}$, such an FBSDE is always solvable. This type of decoupled FBSDE can be characterized by the following:

$$
\left\|b_{y}\right\|_{\infty}=\left\|b_{z}\right\|_{\infty}=\left\|\sigma_{y}\right\|_{\infty}=\left\|\sigma_{z}\right\|_{\infty}=\left\|\gamma_{\xi}\right\|_{\infty}=\left\|\gamma_{y}\right\|_{\infty}=\left\|h_{y}\right\|_{\infty}=0 .
$$

Hence, from (5.12) and (5.13), we see that to have a type (III) Lyapunov operator $P(\cdot)$ of the form (5.14) for such a generator, it suffices to set $\Psi=0, \nu_{2}=1$ and require

$$
\left(\begin{array}{cc}
\nu_{1} I-e^{\kappa_{2} T} h_{\xi}^{*} h_{\xi} & 0 \\
0 & I
\end{array}\right) \gg 0
$$


and

$$
\begin{gathered}
\left(\begin{array}{ccc}
\nu_{1} e^{\kappa_{1}(T-t)}\left(-\kappa_{1} I+b_{x}+b_{x}^{T}+\sigma_{x}^{T} \sigma_{x}\right) & -e^{\kappa_{2} t} g_{x}^{T} & 0 \\
-e^{\kappa_{2} t} g_{x} & -e^{\kappa_{2} t}\left(\kappa_{2} I+g_{y}+g_{y}^{T}\right) & -e^{\kappa_{2} t} g_{z} \\
0 & -e^{\kappa_{2} t} g_{z}^{T} & -e^{\kappa_{2} t} I
\end{array}\right) \\
=-e^{\kappa_{2} t}\left(\begin{array}{ccc}
\nu_{1} e^{\kappa_{1}(T-t)-\kappa_{2} t}\left(\kappa_{1} I-b_{x}-b_{x}^{T}-\sigma_{x}^{T} \sigma_{x}\right) & g_{x}^{T} & 0 \\
g_{x} & \left(\kappa_{2} I+g_{y}+g_{y}^{T}\right) & g_{z} \\
0 & g_{z}^{T} & I
\end{array}\right) \ll 0 .
\end{gathered}
$$

To this end, we can first take $\kappa_{2}>0$ so large that

$$
\kappa_{2}-2\left\|g_{y}\right\|_{\infty}-\left\|g_{z}\right\|_{\infty}^{2}>0
$$

Then take $\kappa_{1}, \nu_{1}$ large enough so that

$$
\left\{\begin{array}{l}
\nu_{1}-e^{\kappa_{2} T}\left\|h_{\xi}\right\|_{\infty}^{2}>0, \\
\nu_{1}\left(\kappa_{1}-2\left\|b_{x}\right\|_{\infty}-\left\|\sigma_{x}\right\|_{\infty}^{2}\right)-\frac{e^{\kappa_{2} T}\left\|g_{x}\right\|_{\infty}^{2}}{\kappa_{2}-2\left\|g_{y}\right\|_{\infty}-\left\|g_{z}\right\|_{\infty}^{2}}>0 .
\end{array}\right.
$$

With such a choice of the constants $\nu_{1}, \nu_{2}, \kappa_{1}, \kappa_{2}>0$ (and with $\Psi=0$ ), $P(\cdot)$ of the form (5.14) is a type (III) Lyapunov operator of the generator.

The second type of decoupled FBSDE is as follows:

$$
\left\{\begin{array}{l}
d X(t)=b(t, X(t), Y(t), Z(t)) d t+\sigma(t, X(t), Y(t), Z(t)) d W(t) \\
d Y(t)=g(t, Y(t), Z(t)) d t+Z(t) d W(t) \\
X(0)=\gamma(Y(0)), \quad Y(T)=\eta
\end{array}\right.
$$

where $\eta \in \mathcal{X}_{m}^{p}$. One can solve the backward equation first and then solve the forward equation. Hence, this case is also always solvable. This type of decoupled FBSDE can be characterized by the following:

$$
\left\|g_{x}\right\|_{\infty}=\left\|\gamma_{\xi}\right\|_{\infty}=\left\|h_{\xi}\right\|_{\infty}=\left\|h_{y}\right\|_{\infty}=0 .
$$

Thus, again taking $\Psi=0$, and taking $\nu_{1}=1$, we need only to require

$$
\left(\begin{array}{cc}
I & 0 \\
0 & \nu_{2} I-e^{\kappa_{1} T} \gamma_{y}^{T} \gamma_{y}
\end{array}\right) \gg 0
$$

and

$$
\begin{gathered}
\left(\begin{array}{ccc}
e^{\kappa_{1}(T-t)}\left(b_{x}+b_{x}^{T}+\sigma_{x}^{T} \sigma_{x}-\kappa_{1} I\right) & e^{\kappa_{1}(T-t)}\left(b_{y}+\sigma_{x}^{T} \sigma_{y}\right) & e^{\kappa_{1}(T-t)}\left(b_{z}+\sigma_{x}^{T} \sigma_{z}\right) \\
e^{\kappa_{1}(T-t)}\left(b_{y}^{T}+\sigma_{y}^{T} \sigma_{x}\right) & e^{\kappa_{1}(T-t)} \sigma_{y}^{T} \sigma_{y}-\nu_{2} e^{\kappa_{2} t}\left(\kappa_{2} I+g_{y}+g_{y}^{T}\right) & e^{\kappa_{1}(T-t)} \sigma_{y}^{T} \sigma_{z}-\nu_{2} e^{\kappa_{2} t} g_{z} \\
e^{\kappa_{1}(T-t)}\left(b_{z}^{T}+\sigma_{z}^{T} \sigma_{x}\right) & e^{\kappa_{1}(T-t)} \sigma_{z}^{T} \sigma_{y}-\nu_{2} e^{\kappa_{2} t} g_{z}^{T} & e^{\kappa_{1}(T-t)} \sigma_{z}^{T} \sigma_{z}-\nu_{2} e^{\kappa_{2} t} I
\end{array}\right) \\
=e^{\kappa_{1}(T-t)}\left[\left(\begin{array}{ccc}
0 & b_{y}+\sigma_{x}^{T} \sigma_{y} & b_{z}+\sigma_{x}^{T} \sigma_{z} \\
b_{y}^{T}+\sigma_{y}^{T} \sigma_{x} & \sigma_{y}^{T} \sigma_{y} & \sigma_{y}^{T} \sigma_{z} \\
b_{z}^{T}+\sigma_{z}^{T} \sigma_{x} & \sigma_{z}^{T} \sigma_{y} & \sigma_{z}^{T} \sigma_{z}
\end{array}\right)\right. \\
\left.-e^{\kappa_{1}(t-T)+\kappa_{2} t}\left(\begin{array}{ccc}
e^{\kappa_{1}(T-t)-\kappa_{2} t}\left(\kappa_{1} I-b_{x}-b_{x}^{T}-\sigma_{x}^{T} \sigma_{x}\right) & 0 & 0 \\
0 & \nu_{2}\left(\kappa_{2} I+g_{y}+g_{y}^{T}\right) & \nu_{2} g_{z} \\
0 & \nu_{2} g_{z}^{T} & \nu_{2} I
\end{array}\right)\right] \ll 0 .
\end{gathered}
$$

Clearly, we can first take $\kappa_{1}, \kappa_{2}>0$ so that

$$
\kappa_{1}-2\left\|b_{x}\right\|_{\infty}-\left\|\sigma_{x}\right\|_{\infty}^{2}>0, \quad \kappa_{2}-2\left\|g_{y}\right\|_{\infty}-\left\|g_{z}\right\|_{\infty}^{2}>0
$$

then take $\nu_{2}>0$ large enough to achieve (6.8)- (6.9). With such a choice of the constants $\nu_{1}, \nu_{2}, \kappa_{1}, \kappa_{2}>0$ (and $\Psi=0$ ), function $P(\cdot)$ of the form (5.14) is a type (III) Lyapunov operator for the corresponding generator. 
6.2. Monotone cases. Let us assume that $m=n$. Suppose there exists a matrix $\Psi \in \mathbb{R}^{n \times n}$ (of full rank) such that the maps

$$
\theta \mapsto\left(\begin{array}{c}
-\Psi^{T} g(t, \theta) \\
-\Psi b(t, \theta) \\
-\Psi \sigma(t, \theta)
\end{array}\right), \quad\left(\begin{array}{c}
\xi \\
y
\end{array}\right) \mapsto\left(\begin{array}{c}
\Psi^{T} h(\xi, y) \\
-\Psi \gamma(\xi, y)
\end{array}\right)
$$

are uniformly monotone; i.e., for some $\delta>0$,

$$
\begin{gathered}
{\left[g\left(t, \theta_{1}\right)-g\left(t, \theta_{2}\right)\right]^{T} \Psi\left(x_{1}-x_{2}\right)+\left[b\left(t, \theta_{1}\right)-b\left(t, \theta_{2}\right)\right]^{T} \Psi^{T}\left(y_{1}-y_{2}\right)} \\
+\left[\sigma\left(t, \theta_{1}\right)-\sigma\left(t, \theta_{2}\right)\right]^{T} \Psi^{T}\left(z_{1}-z_{2}\right) \leq-\delta\left|\theta_{1}-\theta_{2}\right|^{2} \\
\forall t \in[0, T], \theta_{1}, \theta_{2} \in \mathbb{M}, \text { a.s. }
\end{gathered}
$$

and

$$
\begin{aligned}
& \mathbb{E}\left\{\left[h\left(\xi_{1}, y_{1}\right)-h\left(\xi_{2}, y_{2}\right)\right]^{T} \Psi\left(\xi_{1}-\xi_{2}\right)\right\}-\left[\gamma\left(\xi_{1}, y_{1}\right)-\gamma\left(\xi_{2}, y_{2}\right)\right]^{T} \Psi^{T}\left(y_{1}-y_{2}\right) \\
& \geq \delta\left(\mathbb{E}\left|\xi_{1}-\xi_{2}\right|^{2}+\left|y_{1}-y_{2}\right|^{2}\right), \quad \forall \xi_{1}, \xi_{2} \in \mathcal{X}^{2}, y_{1}, y_{2} \in \mathbb{R}^{n} .
\end{aligned}
$$

We refer to the above as the uniform monotone case. Note that (6.11) holds if and only if

$$
\begin{aligned}
& 2 \delta\left(\mathbb{E}|\xi|^{2}+|y|^{2}\right) \leq 2 \mathbb{E}\left[\left(h_{\xi} \xi+h_{y} y\right)^{T} \Psi \xi\right]-2\left[\gamma_{\xi} \xi+\gamma_{y} y\right]^{T} \Psi^{T} y \\
& =\mathbb{E}\left[\xi^{T}\left(h_{\xi}^{*} \Psi+\Psi^{T} h_{\xi}\right) \xi+2 y^{T}\left(h_{y}^{*} \Psi-\Psi \gamma_{\xi}\right) \xi-y^{T}\left(\gamma_{y}^{T} \Psi^{T}+\Psi \gamma_{y}\right) y\right] \\
& =\mathbb{E}\left\{\left(\begin{array}{c}
\xi \\
y
\end{array}\right)^{T}\left(\begin{array}{cc}
\Psi^{T} h_{\xi}+h_{\xi}^{*} \Psi & \Psi^{T} h_{y}-\gamma_{\xi}^{*} \Psi^{T} \\
-\Psi \gamma_{\xi}+h_{y}^{*} \Psi & -\Psi \gamma_{y}-\gamma_{y}^{T} \Psi^{T}
\end{array}\right)\left(\begin{array}{c}
\xi \\
y
\end{array}\right)\right\}, \quad \forall \xi \in \mathcal{X}^{2}, y \in \mathbb{R}^{n} .
\end{aligned}
$$

Similarly, (6.10) holds if and only if

$$
\begin{aligned}
& \theta^{T}\left(\begin{array}{ccc}
\Psi^{T} g_{x}+g_{x}^{T} \Psi & \Psi^{T} g_{y}+b_{x}^{T} \Psi^{T} & \Psi^{T} g_{z}+\sigma_{x}^{T} \Psi^{T} \\
\Psi b_{x}+g_{y}^{T} \Psi & \Psi b_{y}+b_{y}^{T} \Psi^{T} & \Psi b_{z}+\sigma_{y}^{T} \Psi^{T} \\
\Psi \sigma_{x}+g_{z}^{T} \Psi & \Psi \sigma_{y}+b_{z}^{T} \Psi^{T} & \Psi \sigma_{z}+\sigma_{z}^{T} \Psi^{T}
\end{array}\right) \theta \\
& \leq-\delta|\theta|^{2}, \quad \forall t \in[0, T], \theta \in \mathbb{M} \text {, a.s. }
\end{aligned}
$$

Hence, the uniform monotonicity conditions (6.10)-6.11) are equivalent to the following:

$$
\left\{\begin{array}{l}
\left(\begin{array}{ccc}
\Psi^{T} g_{x}+g_{x}^{T} \Psi & b_{x}^{T} \Psi^{T}+\Psi^{T} g_{y} & \Psi^{T} g_{z}+\sigma_{x}^{T} \Psi^{T} \\
\Psi b_{x}+g_{y}^{T} \Psi & \Psi b_{y}+b_{y}^{T} \Psi & \Psi b_{z}+\sigma_{y}^{T} \Psi^{T} \\
\Psi \sigma_{x}+g_{z}^{T} \Psi & \Psi \sigma_{y}+g_{z}^{T} \Psi & \Psi \sigma_{z}+\sigma_{z}^{T} \Psi^{T}
\end{array}\right) \ll 0, \\
\left(\begin{array}{cc}
\Psi^{T} h_{\xi}+h_{\xi}^{*} \Psi & \Psi^{T} h_{y}-\gamma_{\xi}^{*} \Psi^{T} \\
-\Psi \gamma_{\xi}+h_{y}^{*} \Psi & -\Psi \gamma_{y}-\gamma_{y}^{T} \Psi^{T}
\end{array}\right) \gg 0 .
\end{array}\right.
$$

These conditions ensure the existence of a type (III) Lyapunov operator $P(\cdot)$ of the form (5.14) for the generator $(b, \sigma, g, \gamma, h)$, by choosing $\kappa_{1}, \kappa_{2}, \nu_{1}, \nu_{2}>0$ sufficiently small.

An important special case of the above is $\Psi=I_{n}$, for which case the monotonicity conditions become the uniform monotonicity of the following maps:

$$
\theta \mapsto\left(\begin{array}{c}
-g(t, \theta) \\
-b(t, \theta) \\
-\sigma(t, \theta)
\end{array}\right), \quad\left(\begin{array}{c}
\xi \\
y
\end{array}\right) \mapsto\left(\begin{array}{c}
h(\xi, y) \\
-\gamma(\xi, y)
\end{array}\right) .
$$


However, we point out that the above uniform monotonicity conditions are a little too restrictive. To see this, let us look at the classical case ([20]), for which one has

$$
\left\|\gamma_{\xi}\right\|_{\infty}=\left\|\gamma_{y}\right\|_{\infty}=\left\|h_{y}\right\|_{\infty}=0
$$

Then the second condition in (6.14) fails. Note that for the current case, (5.12) becomes

$$
\left(\begin{array}{cc}
\nu_{1} I-\nu_{2} e^{\kappa_{2} T} h_{\xi}^{*} h_{\xi} & 0 \\
0 & \nu_{2} I
\end{array}\right)+\left(\begin{array}{cc}
\Psi^{T} h_{\xi}+h_{\xi}^{*} \Psi & 0 \\
0 & 0
\end{array}\right) \gg 0 .
$$

Hence, we may replace the second condition in (6.14) by the following:

$$
\Psi^{T} h_{\xi}+h_{\xi}^{T} \Psi \geq 0,
$$

since we may choose $\nu_{1}, \nu_{2}, \kappa_{1}, \kappa_{2}>0$ small enough keeping

$$
\nu_{1}>\nu_{2} e^{\kappa_{2} T}\left\|h_{\xi}\right\|_{\infty}^{2} \text {. }
$$

This essentially recovers the case studied in 14. Likewise, if

$$
\left\|h_{\xi}\right\|_{\infty}=\left\|h_{y}\right\|_{\infty}=\left\|\gamma_{\xi}\right\|_{\infty}=0,
$$

then (5.12) becomes

$$
\left(\begin{array}{cc}
\nu_{1} I & 0 \\
0 & \nu_{2} I-\nu_{1} e^{\kappa_{1} T} \gamma_{y}^{T} \gamma_{y}
\end{array}\right)+\left(\begin{array}{cc}
0 & 0 \\
0 & -\Psi \gamma_{y}-\gamma_{y}^{T} \Psi^{T}
\end{array}\right) \gg 0 .
$$

Hence, to ensure the existence of a type (III) Lyapunov operator for such a case, we need only to assume the first condition in (6.14) and

$$
\Psi \gamma_{y}+\gamma_{y}^{T} \Psi^{T} \leq 0,
$$

because (6.19) can be guaranteed by choosing $\nu_{1}, \kappa_{1}>0$ small enough keeping

$$
\nu_{2}>\nu_{1} e^{\kappa_{1} T}\left\|\gamma_{y}\right\|_{\infty}^{2} .
$$

Note that this case is already new.

The above suggests making the following interesting observation. Suppose, say, $\left\|\sigma_{z}\right\|_{\infty}=0$. Then the first condition in (6.14) cannot be true. One can easily cook up various similar cases. In particular, if the FBSDE is decoupled, the first condition in (6.14) must fail. From this, we see that the decoupled case and the uniformly monotone case are mutually exclusive.

Furthermore, we note that in the case $m \neq n$, (6.14) must fail. In fact, if, say, $n>m$, then

$$
\operatorname{rank}\left(\Psi^{T} g_{x}+g_{x}^{T} \Psi\right) \leq m<n .
$$

To summarize the above, we see that while allowing full coupling of FBSDEs, the uniform monotonicity conditions are too restrictive. In the following section, we will carry out some more careful discussions.

6.3. Partially monotone cases. Note that for $(b, \sigma, g, \gamma, h) \in \mathcal{G}^{2}$, due to the uniform Lipschitz condition, for any matrix $\Psi \in \mathbb{R}^{m \times n}$, one can find constants $\alpha_{1}$, $\alpha_{2}, \beta_{1}, \beta_{2}, \beta_{3} \in \mathbb{R}$ (sufficiently negative) such that the maps

$$
\left(\begin{array}{l}
\xi \\
y
\end{array}\right) \mapsto\left(\begin{array}{c}
\Psi^{T} h(\xi, y)-\alpha_{1} \xi \\
-\Psi \gamma(\xi, y)-\alpha_{2} y
\end{array}\right), \quad \theta \mapsto\left(\begin{array}{c}
-\Psi^{T} g(t, \theta)-\beta_{1} x \\
-\Psi b(t, \theta)-\beta_{2} y \\
-\Psi \sigma(t, \theta)-\beta_{3} z
\end{array}\right)
$$


are monotone; i.e.,

$$
\begin{aligned}
& \mathbb{E}\left\{\left[h\left(\xi_{1}, y_{1}\right)-h\left(\xi_{2}, y_{2}\right)\right]^{T} \Psi\left(\xi_{1}-\xi_{2}\right)\right\}-\left[\gamma\left(\xi_{1}, y_{1}\right)-\gamma\left(\xi_{2}, y_{2}\right)\right]^{T} \Psi^{T}\left(y_{1}-y_{2}\right) \\
& \geq \alpha_{1} \mathbb{E}\left|\xi_{1}-\xi_{2}\right|^{2}+\alpha_{2}\left|y_{1}-y_{2}\right|^{2}
\end{aligned}
$$

and

$$
\begin{aligned}
& \quad\left[g\left(t, \theta_{1}\right)-g\left(t, \theta_{2}\right)\right]^{T} \Psi\left(x_{1}-x_{2}\right)+\left[b\left(t, \theta_{1}\right)-b\left(t, \theta_{2}\right)\right]^{T} \Psi^{T}\left(y_{1}-y_{2}\right) \\
& \quad+\left[\sigma\left(t, \theta_{1}\right)-\sigma\left(t, \theta_{2}\right)\right]^{T} \Psi\left(z_{1}-z_{2}\right) \\
& \leq-\beta_{1}\left|x_{1}-x_{2}\right|^{2}-\beta_{2}\left|y_{1}-y_{2}\right|^{2}-\beta_{3}\left|z_{1}-z_{2}\right|^{2}
\end{aligned}
$$

Similar to the discussion of the uniformly monotone case, we see that (6.21) -6.22 ) are equivalent to the following:

$$
\begin{gathered}
\left(\begin{array}{ccc}
\Psi^{T} h_{x}+h_{\xi}^{*} \Psi & \Psi^{T} h_{y}-\gamma_{\xi}^{*} \Psi^{T} \\
-\Psi \gamma_{\xi}+h_{y}^{T} \Psi & -\Psi \gamma_{y}-\gamma_{y}^{T} \Psi^{T}
\end{array}\right) \geq\left(\begin{array}{cc}
\alpha_{1} I & 0 \\
0 & \alpha_{2} I
\end{array}\right) \\
\left(\begin{array}{ccc}
\Psi^{T} g_{x}+g_{x}^{T} \Psi & b_{x}^{T} \Psi^{T}+\Psi^{T} g_{y} & \Psi^{T} g_{z}+\sigma_{x}^{T} \Psi^{T} \\
\Psi b_{x}+g_{y}^{T} \Psi & \Psi b_{y}+b_{y}^{T} \Psi & \Psi b_{z}+\sigma_{y}^{T} \Psi^{T} \\
\Psi \sigma_{x}+g_{z}^{T} \Psi & \Psi \sigma_{y}+b_{z}^{T} \Psi^{T} & \Psi \sigma_{z}+\sigma_{z}^{T} \Psi^{T}
\end{array}\right) \leq-\left(\begin{array}{ccc}
\beta_{1} I & 0 & 0 \\
0 & \beta_{2} I & 0 \\
0 & 0 & \beta_{3} I
\end{array}\right) .
\end{gathered}
$$

Note if all the constants $\alpha_{1}, \alpha_{2}, \beta_{1}, \beta_{2}, \beta_{3}$ are positive (which is possible only when $m=n$ ), conditions (6.21)-6.22) (or 6.23)-6.24) lead to the uniformly monotone case discussed in the previous subsection, which is too restrictive. We now would like to consider the case that not all of the constants $\alpha_{1}, \alpha_{2}, \beta_{1}, \beta_{2}, \beta_{3}$ are positive.

By Proposition 5.2, we see that if (6.23) - 6.24) hold for some $\Psi \in \mathbb{R}^{m \times n}$ and $\alpha_{1}, \alpha_{2}, \beta_{1}, \beta_{2}, \beta_{3} \in \mathbb{R}$, then, provided the following hold,

$$
\left(\begin{array}{cc}
\left(\nu_{1}+\alpha_{1}\right) I-\nu_{1} e^{\kappa_{1} T} \gamma_{\xi}^{*} \gamma_{\xi}-\nu_{2} e^{\kappa_{2} T} h_{\xi}^{*} h_{\xi} & -\nu_{1} e^{\kappa_{1} T} \gamma_{\xi}^{*} \gamma_{y}-\nu_{2} e^{\kappa_{2} T} h_{\xi}^{*} h_{y} \\
-\nu_{1} e^{\kappa_{1} T} \gamma_{y}^{T} \gamma_{\xi}-\nu_{2} e^{\kappa_{2} T} h_{y}^{*} h_{\xi} & \left(\nu_{2}+\alpha_{2}\right) I-\nu_{2} e^{\kappa_{2} T} h_{y}^{*} h_{y}-\nu_{1} e^{\kappa_{1} T} \gamma_{y}^{T} \gamma_{y}
\end{array}\right) \gg 0
$$

and

$$
\begin{aligned}
& \left(\begin{array}{ccc}
\nu_{1} e^{\kappa_{1}(T-t)}\left(b_{x}+b_{x}^{T}+\sigma_{x}^{T} \sigma_{x}-\kappa_{1} I\right) & \nu_{1} e^{\kappa_{1}(T-t)}\left(b_{y}+\sigma_{x}^{T} \sigma_{y}\right)-\nu_{2} e^{\kappa_{2} t} g_{x}^{T} & \nu_{1} e^{\kappa_{1}(T-t)}\left(b_{z}+\sigma_{x}^{T} \sigma_{z}\right) \\
\nu_{1} e^{\kappa_{1}(T-t)}\left(b_{y}^{T}+\sigma_{y}^{T} \sigma_{x}\right)-\nu_{2} e^{\kappa_{2} t} g_{x} & \nu_{1} e^{\kappa_{1}(T-t)} \sigma_{y}^{T} \sigma_{y}-\nu_{2} e^{\kappa_{2} t}\left(\kappa_{2} I+g_{y}+g_{y}^{T}\right) & \nu_{1} e^{\kappa_{1}(T-t)} \sigma_{y}^{T} \sigma_{z}-\nu_{2} e^{\kappa_{2} t} g_{z} \\
\nu_{1} e^{\kappa_{1}(T-t)}\left(b_{z}^{T}+\sigma_{z}^{T} \sigma_{x}\right) & \nu_{1} e^{\kappa_{1}(T-t)} \sigma_{z}^{T} \sigma_{y}-\nu_{2} e^{\kappa_{2} t} g_{z}^{T} & \nu_{1} e^{\kappa_{1}(T-t)} \sigma_{z}^{T} \sigma_{z}-\nu_{2} e^{\kappa_{2} t} I
\end{array}\right) \\
& -\left(\begin{array}{ccc}
\beta_{1} I & 0 & 0 \\
0 & \beta_{2} I & 0 \\
0 & 0 & \beta_{3} I
\end{array}\right) \ll 0,
\end{aligned}
$$

we have the existence of a type (III) Lyapunov operator $P(\cdot)$ of the form (5.14). We now would like to look at conditions under which (6.25)- 6.26) hold. Our first result of this subsection is the following.

Proposition 6.1. Let $(b, \sigma, g, \gamma, h) \in \mathcal{G}^{2}$. Let $\alpha_{1}, \beta_{1}>0, \alpha_{2}, \beta_{2}, \beta_{3} \in \mathbb{R}$, and $\Psi \in \mathbb{R}^{n \times m}$ such that (6.23)- 6.24 ) hold. Let

$$
\lambda_{g} \triangleq \underset{(t, \theta, \omega)}{\operatorname{essinf}}\left[\min \Lambda\left(g_{y}(t, \theta, \omega)+g_{y}(t, \theta, \omega)^{T}-g_{z}(t, \theta, \omega) g_{z}(t, \theta, \omega)^{T}\right)\right] \geq 0
$$

Suppose that the following conditions are satisfied:

$$
\left\|h_{y}\right\|_{\infty}<1
$$




$$
\left\{\begin{array}{l}
\Delta_{h} \triangleq\left[\alpha_{1}\left(1-\left\|h_{y}\right\|_{\infty}^{2}\right)-\alpha_{2}^{-}\left\|h_{\xi}\right\|_{\infty}^{2}\right]^{2}-4 \alpha_{1} \alpha_{2}^{-}\left\|h_{\xi}^{*} h_{y}\right\|_{\infty}^{2}>0 \\
\Delta_{g} \triangleq\left(\beta_{1} \lambda_{g}+\beta_{3}^{-}\left\|g_{x}\right\|_{\infty}^{2}\right)^{2}-4 \beta_{1}\left[\beta_{3}^{-}\left(\lambda_{g}+\left\|g_{z}\right\|_{\infty}^{2}\right)+\beta_{2}^{-}\right]\left\|g_{x}\right\|_{\infty}^{2}>0
\end{array}\right.
$$

and

$$
\mu_{-}^{h} \vee \mu_{-}^{g}<\mu_{+}^{h} \wedge \mu_{+}^{g},
$$

where

$$
\left\{\begin{array}{l}
\mu_{-}^{h}=\frac{2 \alpha_{1} \alpha_{2}^{-}}{\alpha_{1}\left(1-\left\|h_{y}\right\|_{\infty}^{2}\right)+\alpha_{2}^{-}\left\|h_{\xi}\right\|_{\infty}^{2}+\sqrt{\Delta_{h}}}, \\
\mu_{+}^{h}= \begin{cases}\frac{\alpha_{1}\left(1-\left\|h_{y}\right\|_{\infty}^{2}\right)+\alpha_{2}^{-}\left\|h_{x}\right\|_{\infty}^{2}+\sqrt{\Delta_{h}}}{2\left[\left\|h_{\xi}\right\|_{\infty}^{2}\left(1-\left\|h_{y}\right\|_{\infty}^{2}\right)+\left\|h_{\xi}^{*} h_{y}\right\|_{\infty}^{2}\right]}, & \text { if }\left\|h_{\xi}\right\|_{\infty}>0, \\
\infty, & \text { if }\left\|h_{\xi}\right\|_{\infty}=0,\end{cases}
\end{array}\right.
$$

and

$$
\left\{\begin{aligned}
\mu_{-}^{g} & =\frac{2 \beta_{1}\left[\beta_{3}^{-}\left(\lambda_{g}+\left\|g_{z}\right\|_{\infty}^{2}\right)+\beta_{2}^{-}\right]}{\beta_{1} \lambda_{g}+\beta_{3}^{-}\left\|g_{x}\right\|_{\infty}^{2}+\sqrt{\Delta_{g}}}, \\
\mu_{+}^{g} & = \begin{cases}\frac{\beta_{1} \lambda_{g}+\beta_{3}^{-}\left\|g_{x}\right\|_{\infty}^{2}+\sqrt{\Delta_{g}}}{2\left\|g_{x}\right\|_{\infty}^{2}}, & \text { if }\left\|g_{x}\right\|_{\infty}>0, \\
\infty, & \text { if }\left\|g_{x}\right\|_{\infty}=0 .\end{cases}
\end{aligned}\right.
$$

Then the generator $(b, \sigma, g, \gamma, h)$ admits a type (III) Lyapunov operator $P(\cdot)$ of the form (5.14).

In the above, $r^{-}=\max \{0,-r\}$ for any $r \in \mathbb{R}$. Note that when $\alpha_{1}, \beta_{1}>0$, we have the uniform monotonicity of the maps

$$
\xi \mapsto\left(\begin{array}{c}
\Psi^{T} h(\xi, y) \\
-\Psi \gamma(\xi, y)
\end{array}\right), \quad x \mapsto\left(\begin{array}{c}
-\Psi^{T} g(t, x, y, z) \\
-\Psi b(t, x, y, z) \\
-\Psi \sigma(t, x, y, z)
\end{array}\right),
$$

which is possible only if $m \geq n$. Due to the above, we refer to it as a partiallymonotone case.

We point out that in the above proposition, although when $\left\|g_{x}\right\|_{\infty}=0$, the forward and backward equations are decoupled, the initial and terminal conditions may still be coupled, even if $\left\|h_{\xi}\right\|_{\infty}=0$.

We also note that in the above result, there are no explicit restrictions imposed on the following:

$$
\left\|\gamma_{\xi}\right\|_{\infty},\left\|\gamma_{y}\right\|_{\infty},\left\|b_{\theta}\right\|_{\infty},\left\|\sigma_{\theta}\right\|_{\infty},\left\|g_{y}\right\|_{\infty}
$$

which means that these are allowed to be large. This is possible due to the (partially-monotone) condition $\alpha_{1}, \beta_{1}>0$ and the condition $\lambda_{g}>0$.

Although the conditions in the above proposition look complicated, the following corollary tells us the power of the above result.

Corollary 6.2. Let $(b, \sigma, g, \gamma, h) \in \mathcal{G}^{2}$ such that $\alpha_{1}, \beta_{1}>0$ and $\alpha_{2}=\beta_{2}=\beta_{3}=0$. Suppose that

$$
\left\|h_{y}\right\|_{\infty}<1, \quad \lambda_{g}>0 .
$$


Then the generator $(b, \sigma, g, \gamma, h)$ admits a type (III) Lyapunov operator $P(\cdot)$ of the form (5.14).

Proof. Under condition (6.33), we have

$$
\begin{gathered}
\mu_{-}^{h}=\mu_{-}^{g}=0, \\
\mu_{+}^{h}= \begin{cases}\frac{\alpha_{1}\left(1-\left\|h_{y}\right\|_{\infty}^{2}\right)}{\left\|h_{\xi}\right\|_{\infty}^{2}\left(1-\left\|h_{y}\right\|_{\infty}^{2}\right)+\left\|h_{\xi}^{*} h_{y}\right\|_{\infty}^{2}}, & \text { if }\left\|h_{\xi}\right\|_{\infty}>0, \\
\infty, & \text { if }\left\|h_{\xi}\right\|_{\infty}=0,\end{cases}
\end{gathered}
$$

and

$$
\mu_{+}^{g}= \begin{cases}\frac{\beta_{1} \lambda_{g}}{\left\|g_{x}\right\|_{\infty}^{2}}, & \text { if }\left\|g_{x}\right\|_{\infty}>0, \\ \infty, & \text { if }\left\|g_{x}\right\|_{\infty}=0 .\end{cases}
$$

Thus, (6.30) holds and our conclusion follows.

Before proving the above proposition, let us state an elementary lemma whose proof is straightforward.

\section{Lemma 6.3.}

$$
f(\mu)=-a_{2} \mu^{2}+a_{1} \mu-a_{0},
$$

with $a_{1}>0$ and $a_{0}, a_{2} \geq 0$, such that $\Delta \equiv a_{1}^{2}-4 a_{0} a_{2}>0$. Then

$$
\{f>0\} \equiv\{\rho \in \mathbb{R} \mid f(\rho)>0\}=\left(\mu_{-}, \mu_{+}\right),
$$

where

$$
0 \leq \mu_{-} \triangleq \frac{2 a_{0}}{a_{1}+\sqrt{\Delta}}<\mu_{+} \triangleq \begin{cases}\frac{a_{1}+\sqrt{\Delta}}{2 a_{2}}, & a_{2}>0 \\ \infty, & a_{2}=0 .\end{cases}
$$

Proof of Proposition 6.1. By taking $\nu_{1}, \kappa_{2}>0$ sufficiently small, we see that (6.25) and (6.26) are guaranteed by the following:

$$
\left(\begin{array}{cc}
\alpha_{1} I-\nu_{2} h_{\xi}^{*} h_{\xi} & -\nu_{2} h_{\xi}^{*} h_{y} \\
-\nu_{2} h_{y}^{*} h_{\xi} & \left(\nu_{2}+\alpha_{2}\right) I-\nu_{2} h_{y}^{*} h_{y}
\end{array}\right) \gg 0
$$

and

$$
\left(\begin{array}{ccc}
-\beta_{1} I & -\nu_{2} g_{x}^{T} & 0 \\
-\nu_{2} g_{x} & -\nu_{2}\left(g_{y}+g_{y}^{T}\right)-\beta_{2} I & -\nu_{2} g_{z} \\
0 & -\nu_{2} g_{z}^{T} & -\left(\nu_{2}+\beta_{3}\right) I
\end{array}\right) \ll 0 .
$$

We first look at (6.37), which is implied by the following:

$$
\alpha_{1}-\nu_{2}\left\|h_{\xi}\right\|_{\infty}^{2}>0
$$

and

$$
\begin{aligned}
0< & \left(\alpha_{1}-\nu_{2}\left\|h_{\xi}\right\|_{\infty}^{2}\right)\left[\nu_{2}\left(1-\left\|h_{y}\right\|_{\infty}^{2}\right)-\alpha_{2}^{-}\right]-\nu_{2}^{2}\left\|h_{\xi}^{*} h_{y}\right\|_{\infty}^{2} \\
=- & {\left[\left\|h_{\xi}\right\|_{\infty}^{2}\left(1-\left\|h_{y}\right\|_{\infty}^{2}\right)+\left\|h_{\xi}^{*} h_{y}\right\|_{\infty}^{2}\right] \nu_{2}^{2} } \\
& \quad+\left[\alpha_{1}\left(1-\left\|h_{y}\right\|_{\infty}^{2}\right)+\alpha_{2}^{-}\left\|h_{\xi}\right\|_{\infty}^{2}\right] \nu_{2}-\alpha_{1} \alpha_{2}^{-} \\
\equiv- & a_{2} \nu_{2}^{2}+a_{1} \nu_{1}-a_{0} \equiv f_{h}\left(\nu_{2}\right),
\end{aligned}
$$


with $a_{0}, a_{2} \geq 0$, and $a_{1}>0$. Now, by (6.29),

$$
\begin{aligned}
& a_{1}^{2}-4 a_{0} a_{2}=\left[\alpha_{1}\left(1-\left\|h_{y}\right\|_{\infty}^{2}\right)+\alpha_{2}^{-}\left\|h_{\xi}\right\|_{\infty}^{2}\right]^{2} \\
& \quad-4 \alpha_{1} \alpha_{2}^{-}\left[\left\|h_{\xi}\right\|_{\infty}^{2}\left(1-\left\|h_{y}\right\|_{\infty}^{2}\right)+\left\|h_{\xi}^{T} h_{y}\right\|_{\infty}^{2}\right] \\
& =\left[\alpha_{1}\left(1-\left\|h_{y}\right\|_{\infty}^{2}\right)-\alpha_{2}^{-}\left\|h_{\xi}\right\|_{\infty}^{2}\right]^{2}-4 \alpha_{1} \alpha_{2}^{-}\left\|h_{\xi}^{*} h_{y}\right\|_{\infty}^{2} \equiv \Delta_{h}>0 .
\end{aligned}
$$

Hence, by Lemma 6.3, we have

$$
\left\{f_{h}>0\right\}=\left(\mu_{-}^{h}, \mu_{+}^{h}\right),
$$

with $\mu_{+}^{h}$ given by (6.31).

On the other hand, (6.38) is equivalent to the following (recall $\beta_{1}>0$ ):

$$
\left(\begin{array}{cc}
-\beta_{2} I-\nu_{2}\left(g_{y}+g_{y}^{T}\right)+\frac{\nu_{2}^{2}}{\beta_{1}} g_{x} g_{x}^{T} & -\nu_{2} g_{z} \\
-\nu_{2} g_{z}^{T} & \left(-\beta_{3}-\nu_{2}\right) I
\end{array}\right) \ll 0 .
$$

Then, it suffices to find some $\nu_{2}>\beta_{3}^{-}$such that

$$
-\beta_{2} I-\nu_{2}\left(g_{y}+g_{y}^{T}\right)+\frac{\nu_{2}^{2}}{\beta_{1}} g_{x} g_{x}^{T}+\frac{\nu_{2}^{2}}{\nu_{2}+\beta_{3}} g_{z} g_{z}^{T} \ll 0,
$$

which is implied by

$$
\beta_{2}^{-} I-\nu_{2}\left(g_{y}+g_{y}^{T}\right)+\frac{\nu_{2}^{2}}{\beta_{1}} g_{x} g_{x}^{T}+\frac{\nu_{2}^{2}}{\nu_{2}-\beta_{3}^{-}} g_{z} g_{z}^{T} \ll 0 .
$$

This is equivalent to the following:

$$
\begin{aligned}
0 \gg & \beta_{1} \beta_{2}^{-}\left(\nu_{2}-\beta_{3}^{-}\right) I-\beta_{1}\left(\nu_{2}-\beta_{3}^{-}\right) \nu_{2}\left(g_{y}+g_{y}^{T}\right)+\left(\nu_{2}-\beta_{3}^{-}\right) \nu_{2}^{2} g_{x} g_{x}^{T}+\beta_{1} \nu_{2}^{2} g_{z} g_{z}^{T} \\
= & -\beta_{1} \beta_{2}^{-} \beta_{3}^{-} I+\beta_{1} \beta_{2}^{-} \nu_{2} I-\beta_{1}\left(\nu_{2}-\beta_{3}^{-}\right) \nu_{2}\left(g_{y}+g_{y}^{T}\right) \\
& +\left(\nu_{2}-\beta_{3}^{-}\right) \nu_{2}^{2} g_{x} g_{x}^{T}+\beta_{1} \nu_{2}^{2} g_{z} g_{z}^{T} .
\end{aligned}
$$

The above is implied by

$$
\beta_{1}\left(\nu_{2}-\beta_{3}^{-}\right)\left(g_{y}+g_{y}^{T}-g_{z} g_{z}^{T}\right) \gg \beta_{1} \beta_{2}^{-} I+\left(\nu_{2}-\beta_{3}^{-}\right) \nu_{2} g_{x} g_{x}^{T}+\beta_{1} \beta_{3}^{-} g_{z} g_{z}^{T} .
$$

Hence, it suffices to have

$$
\begin{aligned}
& 0<\beta_{1}\left(\nu_{2}-\beta_{3}^{-}\right) \lambda_{g}-\beta_{1} \beta_{2}^{-}-\left(\nu_{2}-\beta_{3}^{-}\right) \nu_{2}\left\|g_{x}\right\|_{\infty}^{2}-\beta_{1} \beta_{3}^{-}\left\|g_{z}\right\|_{\infty}^{2} \\
& =-\left\|g_{x}\right\|_{\infty}^{2} \nu_{2}^{2}+\left(\beta_{1} \lambda_{g}+\beta_{3}^{-}\left\|g_{x}\right\|_{\infty}^{2}\right) \nu_{2}-\beta_{1}\left[\beta_{3}^{-}\left(\lambda_{g}+\left\|g_{z}\right\|_{\infty}^{2}\right)+\beta_{2}^{-}\right] \\
& \equiv-c_{2} \nu_{2}^{2}+c_{1} \nu_{2}-c_{0} \triangleq f_{g}\left(\nu_{2}\right) .
\end{aligned}
$$

By (6.29),

$$
c_{1}^{2}-4 c_{0} c_{2}=\left(\beta_{1} \lambda_{g}+\beta_{3}^{-}\left\|g_{x}\right\|_{\infty}^{2}\right)^{2}-4 \beta_{1}\left[\beta_{3}^{-}\left(\lambda_{g}+\left\|g_{z}\right\|_{\infty}^{2}\right)+\beta_{2}^{-}\right]\left\|g_{x}\right\|_{\infty}^{2} \equiv \Delta_{g}>0 .
$$

Thus, using Lemma 6.3, we obtain

$$
\left\{f_{g}>0\right\}=\left(\mu_{-}^{g}, \mu_{+}^{g}\right),
$$

with $\mu_{ \pm}^{g}$ given by (6.32), and

$$
\begin{aligned}
& 0<\beta_{1}\left(\nu_{2}-\beta_{3}^{-}\right) \lambda_{g}-\beta_{1} \beta_{2}^{-}-\left(\nu_{2}-\beta_{3}^{-}\right) \nu_{2}\left\|g_{x}\right\|_{\infty}^{2}-\beta_{1} \beta_{3}^{-}\left\|g_{z}\right\|_{\infty}^{2} \\
& =-\left\|g_{x}\right\|_{\infty}^{2} \nu_{2}^{2}+\left(\beta_{1} \lambda_{g}+\beta_{3}^{-}\left\|g_{x}\right\|_{\infty}^{2}\right) \nu_{2}-\beta_{1}\left[\beta_{3}^{-}\left(\lambda_{g}+\left\|g_{z}\right\|_{\infty}^{2}\right)+\beta_{2}^{-}\right] \\
& \equiv-c_{2} \nu_{2}^{2}+c_{1} \nu_{2}-c_{0} \triangleq f_{g}\left(\nu_{2}\right) .
\end{aligned}
$$


Next, we claim that

$$
\mu_{-}^{g} \geq \beta_{3}^{-} .
$$

In fact, in the case $\left\|g_{x}\right\|_{\infty}>0, f_{g}(\cdot)$ is a parabola concave downward, and

$$
f_{g}^{\prime}\left(\nu_{2}\right)=\beta_{1} \lambda_{g}+\beta_{3}^{-}\left\|g_{x}\right\|_{\infty}^{2}-2\left\|g_{x}\right\|_{\infty}^{2} \nu_{2}=0
$$

leads to (noting (6.28) $)$

$$
\nu_{2}=\frac{\beta_{1} \lambda_{g}+\beta_{3}^{-}\left\|g_{x}\right\|_{\infty}^{2}}{2\left\|g_{x}\right\|_{\infty}^{2}}=\frac{\beta_{1} \lambda_{g}}{2\left\|g_{x}\right\|_{\infty}^{2}}+\frac{\beta_{3}^{-}}{2}>\beta_{3}^{-},
$$

at which $f_{g}(\cdot)$ attains its (positive) maximum. Clearly, the maximum point of $f_{g}(\cdot)$ is in the interval $\left(\mu_{-}^{g}, \mu_{+}^{g}\right)$. Moreover,

$$
\begin{aligned}
f_{g}\left(\beta_{3}^{-}\right) & =-\left\|g_{x}\right\|_{\infty}^{2}\left(\beta_{3}^{-}\right)^{2}+\left(\beta_{1} \lambda_{g}+\beta_{3}^{-}\left\|g_{x}\right\|_{\infty}^{2}\right) \beta_{3}^{-}-\beta_{1}\left[\beta_{3}^{-}\left(\lambda_{g}+\left\|g_{z}\right\|_{\infty}^{2}\right)+\beta_{2}^{-}\right] \\
& =-\beta_{1} \beta_{3}^{-}\left\|g_{z}\right\|_{\infty}^{2}-\beta_{1} \beta_{2}^{-} \leq 0 .
\end{aligned}
$$

Thus, noting (6.40), we must have (6.39). Now in the case $\left\|g_{x}\right\|_{\infty}=0$, we have

$$
\mu_{-}^{g}=\frac{\beta_{3}^{-}\left(\lambda_{g}+\left\|g_{z}\right\|_{\infty}^{2}\right)+\beta_{2}^{-}}{\lambda_{g}} \geq \beta_{3}^{-} .
$$

Consequently,

$$
\left\{\nu_{2}>\beta_{3}^{-} \mid f_{g}\left(\nu_{2}\right)>0\right\}=\left\{f_{g}>0\right\}=\left(\mu_{-}^{g}, \mu_{+}^{g}\right) .
$$

Finally, condition (6.30) tells us that

$$
\left\{f_{h}>0\right\} \cap\left\{f_{g}>0\right\} \neq \phi,
$$

which means that one can find a $\nu_{2}>0$ such that (6.25) - 6.26$)$ hold. Hence, a type (III) Lyapunov operator $P(\cdot)$ of the form (5.14) exists.

We now present the second result of this subsection.

Proposition 6.4. Let $(b, \sigma, g, \gamma, h) \in \mathcal{G}^{2}$. Let $\alpha_{2}, \beta_{2}, \beta_{3}>0, \alpha_{1}, \beta_{1} \in \mathbb{R}$, and $\Psi \in \mathbb{R}^{n \times m}$ such that (6.23)-(6.24) hold. Let

$$
\lambda_{b \sigma}=\underset{(t, \theta, \omega)}{\operatorname{essinf}}\left[\min \Lambda\left(-b_{x}(t, \theta, \omega)-b_{x}(t, \theta, \omega)^{T}-\sigma_{x}(t, \theta, \omega)^{T} \sigma_{x}(t, \theta, \omega)\right)\right]>0,
$$

and denote

$$
a=\lambda_{b \sigma}\left(\left\|\sigma_{y}\right\|_{\infty}^{2}+\left\|\sigma_{z}\right\|_{\infty}^{2}\right)+\left\|b_{y}+\sigma_{x}^{T} \sigma_{y}\right\|_{\infty}^{2}+\left\|b_{z}+\sigma_{x}^{T} \sigma_{z}\right\|_{\infty}^{2} .
$$

Suppose the following hold:

$$
\left\{\begin{array}{l}
\Delta_{\gamma}=\left[\alpha_{2}\left(1-\left\|\gamma_{\xi}\right\|_{\infty}^{2}\right)-\alpha_{1}^{-}\left\|\gamma_{y}\right\|_{\infty}^{2}\right]^{2}-4 \alpha_{1}^{-} \alpha_{2}\left\|\gamma_{\xi}^{*} \gamma_{y}\right\|_{\infty}^{2}>0 \\
\Delta_{b \sigma}=\left[\beta_{2} \lambda_{b \sigma}+\beta_{1}^{-}\left(\left\|\sigma_{y}\right\|_{\infty}^{2}+\left\|\sigma_{z}\right\|_{\infty}^{2}\right)\right]^{2}-4 \beta_{1}^{-} \beta_{2} a>0
\end{array}\right.
$$

and

$$
\mu_{-}^{\gamma} \vee \mu_{-}^{b \sigma}<\mu_{+}^{\gamma} \wedge \mu_{+}^{b \sigma}
$$


where

$$
\left\{\begin{array}{l}
\mu_{-}^{\gamma}=\frac{2 \alpha_{1}^{-} \alpha_{2}}{\alpha_{2}\left(1-\left\|\gamma_{\xi}\right\|_{\infty}^{2}\right)+\alpha_{1}^{-}\left\|\gamma_{y}\right\|_{\infty}^{2}+\sqrt{\Delta_{\gamma}}}, \\
\mu_{+}^{\gamma}= \begin{cases}\frac{\alpha_{2}\left(1-\left\|\gamma_{\xi}\right\|_{\infty}^{2}\right)+\alpha_{1}^{-}\left\|\gamma_{y}\right\|_{\infty}^{2}+\sqrt{\Delta_{\gamma}}}{2\left[\left\|\gamma_{y}\right\|_{\infty}^{2}\left(1-\left\|\gamma_{\xi}\right\|_{\infty}^{2}\right)+\left\|\gamma_{\xi}^{*} \gamma_{y}\right\|_{\infty}^{2}\right]}, & \text { if }\left\|\gamma_{y}\right\|_{\infty}>0, \\
\infty, & \text { if }\left\|\gamma_{y}\right\|_{\infty}=0,\end{cases}
\end{array}\right.
$$

and

$$
\left\{\begin{array}{l}
\mu_{-}^{b \sigma}=\frac{2 \beta_{1}^{-}\left(\beta_{2} \wedge \beta_{3}\right)}{\left(\beta_{2} \wedge \beta_{3}\right) \lambda_{b \sigma}+\beta_{1}^{-}\left(\left\|\sigma_{y}\right\|_{\infty}^{2}+\left\|\sigma_{z}\right\|_{\infty}^{2}\right)+\sqrt{\Delta_{b \sigma}}}, \\
\mu_{+}^{b \sigma}= \begin{cases}\frac{\left(\beta_{2} \wedge \beta_{3}\right) \lambda_{b \sigma}+\beta_{1}^{-}\left(\left\|\sigma_{y}\right\|_{\infty}^{2}+\left\|\sigma_{z}\right\|_{\infty}^{2}\right)+\sqrt{\Delta_{b \sigma}}}{2 a}, & \text { if } a>0, \\
\infty, & \text { if } a=0 .\end{cases}
\end{array}\right.
$$

Then the generator $(b, \sigma, g, \gamma, h)$ admits a type (III) Lyapunov operator $P(\cdot)$ of the form (5.14).

It is easy to see that in the current case, the maps

$$
y \mapsto\left(\begin{array}{c}
\Psi^{T} h(\xi, y) \\
-\Psi \gamma(\xi, y)
\end{array}\right), \quad\left(\begin{array}{c}
y \\
z
\end{array}\right) \mapsto\left(\begin{array}{c}
-\Psi^{T} g(t, x, y, z) \\
-\Psi b(t, x, y, z) \\
-\Psi \sigma(t, x, y, z)
\end{array}\right)
$$

are uniformly monotone, which is possible only if $n \geq m$. Thus, this is another type of a partially-monotone case. We point out that even if $m=n$, when (6.23)-(6.24) hold for some $\alpha_{2}, \beta_{2}, \beta_{3}>0, \alpha_{1}, \beta_{1}<0$, and $\Psi \in \mathbb{R}^{m \times n}$, it does not mean that (6.23) - 6.24) hold for some $\alpha_{2}, \beta_{2}, \beta_{3}<0, \alpha_{1}, \beta_{1}>0$, and $-\Psi \in \mathbb{R}^{m \times n}$, because when we replace $\Psi$ by $-\Psi$, the inequalities in (6.23) - (6.24) will be reversed. Hence, the situation considered in Proposition 6.4 cannot be reduced to that considered in Proposition 6.1. Next, we note that in Proposition 6.4, $a=0$ if and only if

$$
\left\|b_{y}\right\|_{\infty}=\left\|b_{z}\right\|_{\infty}=\left\|\sigma_{y}\right\|_{\infty}=\left\|\sigma_{z}\right\|_{\infty}=0 .
$$

When this happens, the forward and backward equations are decoupled. But, the initial and terminal conditions may still be coupled, even if $\left\|\gamma_{y}\right\|_{\infty}=0$.

Similar to Proposition 6.1, in the above, we do not have explicit restrictions on the following:

$$
\left\|h_{\xi}\right\|_{\infty},\left\|h_{y}\right\|_{\infty},\left\|b_{x}\right\|_{\infty},\left\|g_{\theta}\right\|_{\infty},
$$

due to the partial monotonicity condition $\alpha_{2}, \beta_{2}, \beta_{3}>0$, and condition $\lambda_{b \sigma}>0$. Also, similar to Corollary 6.2, we have the following.

Corollary 6.5. Let $(b, \sigma, g, \gamma, h) \in \mathcal{G}^{2}$ such that $\alpha_{2}, \beta_{2}, \beta_{3}>0$ and $\alpha_{1}=\beta_{1}=0$. Suppose that

$$
\left\|\gamma_{\xi}\right\|_{\infty}<1, \quad \lambda_{b \sigma}>0
$$

Then the generator $(b, \sigma, g, \gamma, h)$ admits a type (III) Lyapunov operator $P(\cdot)$ of the form (5.14). 
Proof. In the current case, we have

$$
\begin{gathered}
\mu_{-}^{\gamma}=\mu_{-}^{b \sigma}=0, \\
\mu_{+}^{\gamma}= \begin{cases}\frac{\alpha_{2}\left(1-\left\|\gamma_{\xi}\right\|_{\infty}^{2}\right)}{\left\|\gamma_{y}\right\|_{\infty}^{2}\left(1-\left\|\gamma_{\xi}\right\|_{\infty}^{2}\right)+\left\|\gamma_{\xi}^{*} \gamma_{y}\right\|_{\gamma}^{2}}, & \text { if }\left\|\gamma_{y}\right\|_{\gamma}>0, \\
\infty, & \text { if }\left\|\gamma_{y}\right\|_{\infty}=0,\end{cases}
\end{gathered}
$$

and

$$
\mu_{+}^{b \sigma}= \begin{cases}\frac{\left(\beta_{2} \wedge \beta_{3}\right) \lambda_{b \sigma}}{a}, & \text { if } a>0 \\ \infty, & \text { if } a=0 .\end{cases}
$$

Hence, our conclusion follows.

We now present a proof of Proposition 6.4, which has a similar flavor to that of Proposition 6.1.

Proof. By taking $\kappa_{1}, \nu_{2}>0$ sufficiently small, we see that (6.25) and (6.26) are implied by the following:

$$
\left(\begin{array}{cc}
\left(\nu_{1}+\alpha_{1}\right) I-\nu_{1} \gamma_{\xi}^{*} \gamma_{\xi} & -\nu_{1} \gamma_{\xi}^{*} \gamma_{y} \\
-\nu_{1} \gamma_{y}^{T} \gamma_{\xi} & \alpha_{2} I-\nu_{1} \gamma_{y}^{T} \gamma_{y}
\end{array}\right) \gg 0
$$

and

$$
\left(\begin{array}{ccc}
\nu_{1}\left(b_{x}+b_{x}^{T}+\sigma_{x}^{T} \sigma_{x}\right)-\beta_{1} I & \nu_{1}\left(b_{y}+\sigma_{x}^{T} \sigma_{y}\right) & \nu_{1}\left(b_{z}+\sigma_{x}^{T} \sigma_{z}\right) \\
\nu_{1}\left(b_{y}^{T}+\sigma_{y}^{T} \sigma_{x}\right) & \nu_{1} \sigma_{y}^{T} \sigma_{y}-\beta_{2} I & \nu_{1} \sigma_{y}^{T} \sigma_{z} \\
\nu_{1}\left(b_{z}^{T}+\sigma_{z}^{T} \sigma_{x}\right) & \nu_{1} \sigma_{z}^{T} \sigma_{y} & \nu_{1} \sigma_{z}^{T} \sigma_{z}-\beta_{3} I
\end{array}\right) \ll 0,
$$

First, let us look at (6.50), which is implied by the the following:

$$
\nu_{1}\left(1-\left\|\gamma_{\xi}\right\|_{\infty}^{2}\right)-\alpha_{1}^{-}>0, \quad \alpha_{2}-\nu_{1}\left\|\gamma_{y}\right\|_{\infty}^{2}>0
$$

and

$$
\begin{aligned}
0 & <\left[\nu_{1}\left(1-\left\|\gamma_{\xi}\right\|_{\infty}^{2}\right)-\alpha_{1}^{-}\right]\left(\alpha_{2}-\nu_{1}\left\|\gamma_{y}\right\|_{\infty}^{2}\right)-\left\|\gamma_{\xi}^{*} \gamma_{y}\right\|_{\infty}^{2} \nu_{1}^{2} \\
& =-\left[\left\|\gamma_{y}\right\|_{\infty}^{2}\left(1-\left\|\gamma_{\xi}\right\|_{\infty}^{2}\right)+\left\|\gamma_{\xi}^{*} \gamma_{y}\right\|_{\infty}^{2}\right] \nu_{1}^{2}+\left[\alpha_{2}\left(1-\left\|\gamma_{\xi}\right\|_{\infty}^{2}\right)+\alpha_{1}^{-}\left\|\gamma_{y}\right\|_{\infty}^{2}\right] \nu_{1}-\alpha_{1}^{-} \alpha_{2} \\
& \equiv-a_{2} \nu_{1}^{2}+a_{1} \nu_{1}-a_{0} \equiv f_{\gamma}\left(\nu_{1}\right) .
\end{aligned}
$$

By (6.44),

$$
\begin{aligned}
& a_{1}^{2}-4 a_{0} a_{2} \\
& =\left[\alpha_{2}\left(1-\left\|\gamma_{\xi}\right\|_{\infty}^{2}\right)+\alpha_{1}^{-}\left\|\gamma_{y}\right\|_{\infty}^{2}\right]^{2}-4 \alpha_{1}^{-} \alpha_{2}\left[\left\|\gamma_{y}\right\|_{\infty}^{2}\left(1-\left\|\gamma_{\xi}\right\|_{\infty}^{2}\right)+\left\|\gamma_{\xi}^{*} \gamma_{y}\right\|_{\infty}^{2}\right] \\
& =\left[\alpha_{2}\left(1-\left\|\gamma_{\xi}\right\|_{\infty}^{2}\right)-\alpha_{1}^{-}\left\|\gamma_{y}\right\|_{\infty}^{2}\right]^{2}-4 \alpha_{1}^{-} \alpha_{2}\left\|\gamma_{\xi}^{*} \gamma_{y}\right\|_{\infty}^{2}=\Delta_{\gamma}>0 .
\end{aligned}
$$

Thus, by Lemma 6.3 , we have

$$
\left\{f_{\gamma}>0\right\}=\left(\mu_{-}^{\gamma}, \mu_{+}^{\gamma}\right)
$$

with $\mu_{ \pm}^{\gamma}$ given by (6.46).

Now, we look at (6.51). Note that

$$
\begin{aligned}
& \lambda_{b \sigma} \nu_{1}-\beta_{1}^{-}-\nu_{1}^{2}\left\|\left(\begin{array}{c}
b_{y}^{T}+\sigma_{y}^{T} \sigma_{x} \\
b_{z}^{T}+\sigma_{z}^{T} \sigma_{x}
\end{array}\right)\left[\left(\beta_{2} \wedge \beta_{3}\right) I-\nu_{1}\left(\begin{array}{c}
\sigma_{y}^{T} \\
\sigma_{z}^{T}
\end{array}\right)\left(\begin{array}{ll}
\sigma_{y} & \sigma_{z}
\end{array}\right)\right]^{-1}\left(\begin{array}{c}
b_{y}^{T}+\sigma_{y}^{T} \sigma_{x} \\
b_{z}^{T}+\sigma_{z}^{T} \sigma_{x}
\end{array}\right)^{T}\right\| \\
& \leq \lambda_{b \sigma} \nu_{1}-\beta_{1}^{-}-\frac{\nu_{1}^{2}\left(\left\|b_{y}+\sigma_{x}^{T} \sigma_{y}\right\|_{\infty}^{2}+\left\|b_{z}+\sigma_{x}^{T} \sigma_{z}\right\|_{\infty}^{2}\right)}{\left(\beta_{2} \wedge \beta_{3}\right)-\nu_{1}\left(\left\|\sigma_{y}\right\|_{\infty}^{2}+\left\|\sigma_{z}\right\|_{\infty}^{2}\right)} .
\end{aligned}
$$


Thus, it suffices to have

$$
\lambda_{b \sigma} \nu_{1}-\beta_{1}^{-}>0
$$

and

$$
\begin{aligned}
0< & \left(\lambda_{b \sigma} \nu_{1}-\beta_{1}^{-}\right)\left[\left(\beta_{2} \wedge \beta_{2}\right)-\nu_{1}\left(\left\|\sigma_{y}\right\|_{\infty}^{2}+\left\|\sigma_{z}\right\|_{\infty}^{2}\right)\right] \\
& -\nu_{1}^{2}\left(\left\|b_{y}+\sigma_{x}^{T} \sigma_{y}\right\|_{\infty}^{2}+\left\|b_{z}+\sigma_{x}^{T} \sigma_{z}\right\|_{\infty}^{2}\right) \\
= & -\left[\lambda_{b \sigma}\left(\left\|\sigma_{y}\right\|_{\infty}^{2}+\left\|\sigma_{z}\right\|_{\infty}^{2}\right)+\left\|b_{y}+\sigma_{x}^{T} \sigma_{y}\right\|_{\infty}^{2}+\left\|b_{z}+\sigma_{x}^{T} \sigma_{z}\right\|_{\infty}^{2}\right] \nu_{1}^{2} \\
& +\nu_{1}\left[\beta_{2} \lambda_{b \sigma}+\beta_{1}^{-}\left(\left\|\sigma_{y}\right\|_{\infty}^{2}+\left\|\sigma_{z}\right\|_{\infty}^{2}\right)\right]-\beta_{1}^{-}\left(\beta_{2} \wedge \beta_{3}\right) \\
\equiv & -c_{2} \nu_{1}^{2}+c_{1} \nu_{1}-c_{0} \equiv f_{b \sigma}\left(\nu_{1}\right) .
\end{aligned}
$$

Similar to the above discussion, we have that

$$
\left\{f_{b \sigma}>0\right\}=\left(\mu_{-}^{b \sigma}, \mu_{+}^{b \sigma}\right),
$$

where $\mu_{ \pm}^{b \sigma}$ are given by (6.47). Then condition (6.45) implies that

$$
\left\{f_{\gamma}>0\right\} \cap\left\{f_{b \sigma}>0\right\} \neq \phi
$$

which leads to the existence of a Lyapunov operator $P(\cdot)$ of the form (5.14).

From the above proofs, we see that under the conditions of Proposition 6.1, the existing type (III) Lyapunov operator is essentially of the form $\left(\begin{array}{cc}0 & \Psi^{T} \\ \Psi & -\nu_{2} I\end{array}\right)$, and the existing type (III) Lyapunov operator under the conditions of Proposition 6.4 is essentially of the form $\left(\begin{array}{cc}\nu_{1} I & \Psi^{T} \\ \Psi & 0\end{array}\right)$.

6.4. Diagonal Lyapunov operators. It is seen that when an FBSDE is decoupled, we can construct a diagonal type (III) Lyapunov operator (by which we mean that $P(\cdot)$ is diagonal). On the other hand, from the previous two subsections, we see that uniform monotonicity and/or partial monotonicity conditions help us to construct type (III) Lyapunov operators (not necessarily diagonal). However, in general, even partial monotonicity conditions could not be expected. In other words, for any matrix $\Psi \in \mathbb{R}^{m \times n}$, one might not have (6.23) -6.24) for either $\alpha_{1}, \beta_{1}>0$, or $\alpha_{2}, \beta_{2}, \beta_{3}>0$ (uniformly in $(\xi, y) \in \mathcal{X}_{n}^{2} \times \mathbb{R}^{m}$ and in $(t, \theta) \in[0, T] \times \mathbb{M}$, respectively). Hence, if we still want to find a type (III) Lyapunov operator $P(\cdot)$ of the form (5.14), we probably would have to choose $\Psi=0$, which leads to $P(\cdot)$ being diagonal. Then a natural question is: Do we have coupled FBSDEs that admit Lyapunov operators of the form (5.14) with $\Psi=0$ ?

Our first result of this subsection is the following.

Proposition 6.6. Let $(b, \sigma, g, \gamma, h) \in \mathcal{G}^{2}$. Suppose that

$$
1-\left\|\gamma_{\xi}\right\|_{\infty}^{2}-\left\|h_{y}\right\|_{\infty}^{2}-2\left\|h_{\xi}\right\|_{\infty}\left\|\gamma_{y}\right\|_{\infty}>0
$$

and

$$
\begin{aligned}
& 1-\left\|\gamma_{\xi}\right\|_{\infty}^{2}-\left\|h_{y}\right\|_{\infty}^{2}+\sqrt{\left(1-\left\|\gamma_{\xi}\right\|_{\infty}^{2}-\left\|h_{y}\right\|_{\infty}^{2}\right)^{2}-4\left\|h_{\xi}\right\|_{\infty}^{2}\left\|\gamma_{y}\right\|_{\infty}^{2}} \\
& >2\left\|h_{\xi}\right\|_{\infty}^{2}\left\|\sigma_{z}\right\|_{\infty}^{2} .
\end{aligned}
$$

Then there exists a $\lambda_{0}>0$ such that $(b, \sigma, g, \gamma, h)$ admits a diagonal type (III) Lyapunov operator $P(\cdot)$ as long as

$$
\lambda_{b}^{0}, \lambda_{g}^{0} \geq \lambda_{0},
$$


where

$$
\left\{\begin{array}{l}
\lambda_{b}^{0}=\underset{(t, \theta, \omega)}{\operatorname{essinf}}\left[\max \Lambda\left(-b_{x}(t, \theta, \omega)-b_{x}(t, \theta, \omega)^{T}\right)\right], \\
\lambda_{g}^{0}=\underset{(t, \theta, \omega)}{\operatorname{essinf}}\left[\min \Lambda\left(g_{y}(t, \theta, \omega)+g_{y}(t, \theta, \omega)^{T}\right)\right] .
\end{array}\right.
$$

Note that for the classical case:

$$
\left\|\gamma_{\xi}\right\|_{\infty}=\left\|\gamma_{y}\right\|_{\infty}=\left\|h_{y}\right\|_{\infty}=0
$$

Condition (6.52) is always true, and condition (6.53) becomes

$$
\left\|h_{\xi}\right\|_{\infty}\left\|\sigma_{z}\right\|_{\infty}<1
$$

which will always be the case if, in addition, $\left\|\sigma_{z}\right\|_{\infty}=0$.

Before proving the above result, let us make an observation. Suppose

$$
\left\|\gamma_{\xi}\right\|_{\infty}=\left\|h_{y}\right\|_{\infty}=0
$$

Let (1.1) be well posed with $\Theta(\cdot) \equiv(X(\cdot), Y(\cdot), Z(\cdot)) \in \mathcal{M}^{p}[0, T]$ being its unique adapted solution. For any $\mu \in \mathbb{R}$, the process defined by (6.58)

$$
\widetilde{\Theta}(t) \equiv(\widetilde{X}(t), \widetilde{Y}(t), \widetilde{Z}(t)) \equiv e^{\mu t} \Theta(t) \equiv\left(e^{\mu t} X(t), e^{\mu t} Y(t), e^{\mu t} Z(t)\right), t \in[0, T],
$$

is the unique adapted solution of the following FBSDE:

$$
\left\{\begin{array}{l}
d \widetilde{X}(t)=\widetilde{b}(t, \widetilde{\Theta}(t)) d t+\widetilde{\sigma}(t, \widetilde{\Theta}(t)) d W(t) \\
d \widetilde{Y}(t)=\widetilde{g}(t, \widetilde{\Theta}(t)) d t+\widetilde{Z}(t) d W(t) \\
\widetilde{X}(0)=\widetilde{\gamma}(\widetilde{Y}(0)), \quad \widetilde{Y}(T)=\widetilde{h}(\widetilde{X}(T))
\end{array}\right.
$$

with

$$
\left\{\begin{array}{l}
\widetilde{b}(t, \widetilde{\theta})=\mu \widetilde{x}+e^{\mu t} b\left(t, e^{-\mu t} \widetilde{\theta}\right), \quad \widetilde{\sigma}(t, \widetilde{\theta})=\mu \widetilde{y}+e^{\mu t} \sigma\left(t, e^{-\mu t} \widetilde{\theta}\right), \\
\widetilde{g}(t, \widetilde{\theta})=e^{\mu t} g\left(t, e^{-\mu t} \widetilde{\theta}\right), \quad \widetilde{\gamma}(\widetilde{y})=\gamma(\widetilde{y}), \quad \widetilde{h}(\widetilde{x})=e^{\mu T} h\left(e^{-\mu T} \widetilde{x}\right) .
\end{array}\right.
$$

Thus, we have that

$$
\left\{\begin{array}{l}
\widetilde{b}_{\widetilde{x}}(t, \widetilde{\theta})=\mu I+b_{x}\left(t, e^{-\mu t} \widetilde{\theta}\right), \quad \widetilde{b}_{\widetilde{y}}(t, \widetilde{\theta})=b_{y}\left(t, e^{-\mu t} \widetilde{\theta}\right), \quad \widetilde{b}_{\widetilde{z}}(t, \widetilde{\theta})=b_{z}\left(t, e^{-\mu t} \widetilde{\theta}\right) \\
\widetilde{\sigma}_{\widetilde{x}}(t, \widetilde{\theta})=\sigma_{x}\left(t, e^{-\mu t} \widetilde{\theta}\right), \quad \widetilde{\sigma}_{\widetilde{y}}(t, \widetilde{\theta})=\sigma_{y}\left(t, e^{-\mu t} \widetilde{\theta}\right), \quad \widetilde{\sigma}_{\widetilde{z}}(t, \widetilde{\theta})=\sigma_{z}\left(t, e^{-\mu t} \widetilde{\theta}\right) \\
\widetilde{g}_{\widetilde{x}}(t, \widetilde{\theta})=g_{x}\left(t, e^{-\mu t} \widetilde{\theta}\right), \quad \widetilde{g}_{\widetilde{y}}(t, \widetilde{\theta})=\mu I+g_{y}\left(t, e^{-\mu t} \widetilde{\theta}\right), \quad \widetilde{g}_{\widetilde{z}}(t, \widetilde{\theta})=g_{z}\left(t, e^{-\mu t} \widetilde{\theta}\right)
\end{array}\right.
$$

and

$$
\widetilde{\gamma}_{\widetilde{y}}(\widetilde{y})=\gamma_{y}(y), \quad \widetilde{h}_{\widetilde{x}}(\widetilde{x})=h_{x}\left(e^{-\mu T} \widetilde{x}\right)
$$

Consequently,

$$
\begin{aligned}
\widetilde{\lambda}_{b}^{0} & \triangleq \underset{(t, \widetilde{\theta}, \omega)}{\operatorname{essinf}}\left[\max \Lambda\left(-\widetilde{b}_{\widetilde{x}}(t, \widetilde{\theta}, \omega)-\widetilde{b}_{\widetilde{x}}(t, \theta, \omega)^{T}\right)\right] \\
& =-\mu+\underset{(t, \theta, \omega)}{\operatorname{essinf}}\left[\max \Lambda\left(-b_{x}(t, \theta, \omega)-b_{x}(t, \theta, \omega)^{T}\right)\right]=-\mu+\lambda_{b}^{0},
\end{aligned}
$$

and

$$
\begin{aligned}
\widetilde{\lambda}_{g}^{0} & \triangleq \underset{(t, \tilde{\theta}, \omega)}{\operatorname{essinf}}\left[\min \Lambda\left(\widetilde{g}_{\widetilde{y}}(t, \widetilde{\theta}, \omega)+\widetilde{g}_{\widetilde{y}}(t, \widetilde{\theta}, \omega)^{T}\right)\right] \\
& =\mu+\underset{(t, \theta, \omega)}{\operatorname{essinf}}\left[\min \Lambda\left(g_{y}(t, \theta, \omega)+g_{y}(t, \theta, \omega)^{T}\right)\right]=\mu+\lambda_{g}^{0} .
\end{aligned}
$$


As a result,

$$
\lambda_{b}^{0}+\lambda_{g}^{0}=\widetilde{\lambda}_{b}^{0}+\widetilde{\lambda}_{g}^{0} .
$$

This shows that transformation (6.58) cannot simultaneously make $\lambda_{b}$ and $\lambda_{g}$ large. Thus, condition (6.54) is sort of intrinsic.

Proof of Proposition 6.6. By taking $\kappa_{1}, \kappa_{2}>0$ sufficiently small, and $\nu_{1}=1$, we see that a diagonal Lyapunov operator exists if for some $\nu_{2}>0$,

$$
\left(\begin{array}{cc}
I-\gamma_{\xi}^{*} \gamma_{\xi}-\nu_{2} h_{\xi}^{*} h_{\xi} & -\gamma_{\xi}^{*} \gamma_{y}-\nu_{2} h_{\xi}^{*} h_{y} \\
-\gamma_{y}^{T} \gamma_{\xi}-\nu_{2} h_{y}^{*} h_{x} & \nu_{2}\left(I-h_{y}^{*} h_{y}\right)-\gamma_{y}^{T} \gamma_{y}
\end{array}\right) \gg 0
$$

and

$$
\left(\begin{array}{ccc}
b_{x}+b_{x}^{T}+\sigma_{x}^{T} \sigma_{x} & b_{y}+\sigma_{x}^{T} \sigma_{y}-\nu_{2} g_{x}^{T} & b_{z}+\sigma_{x}^{T} \sigma_{z} \\
b_{y}^{T}+\sigma_{y}^{T} \sigma_{x}-\nu_{2} g_{x} & \sigma_{y}^{T} \sigma_{y}-\nu_{2}\left(g_{y}+g_{y}^{T}\right) & \sigma_{y}^{T} \sigma_{z}-\nu_{2} g_{z} \\
b_{z}^{T}+\sigma_{z}^{T} \sigma_{x} & \sigma_{z}^{T} \sigma_{y}-\nu_{2} g_{z}^{T} & \sigma_{z}^{T} \sigma_{z}-\nu_{2} I
\end{array}\right) \ll 0 .
$$

Clearly, (6.59) holds if

$$
\left\{\begin{array}{l}
1-\left\|\gamma_{\xi}\right\|_{\infty}^{2}-\nu_{2}\left\|h_{\xi}\right\|_{\infty}^{2}>0 \\
\nu_{2}\left(1-\left\|h_{y}\right\|_{\infty}^{2}\right)-\left\|\gamma_{y}\right\|_{\infty}^{2}>0
\end{array}\right.
$$

and

$$
\begin{aligned}
0<(1 & \left.-\left\|\gamma_{\xi}\right\|_{\infty}^{2}-\nu_{2}\left\|h_{\xi}\right\|_{\infty}^{2}\right)\left(\nu_{2}\left(1-\left\|h_{y}\right\|_{\infty}^{2}\right)-\left\|\gamma_{y}\right\|_{\infty}^{2}\right) \\
& -\left(\left\|\gamma_{\xi}^{*} \gamma_{y}\right\|_{\infty}+\nu_{2}\left\|h_{\xi}^{*} h_{y}\right\|_{\infty}\right)^{2} \\
=- & {\left[\left\|h_{\xi}\right\|_{\infty}^{2}\left(1-\left\|h_{y}\right\|_{\infty}^{2}\right)+\left\|h_{\xi}^{*} h_{y}\right\|_{\infty}^{2}\right] \nu_{2}^{2}-\left[\left(1-\left\|\gamma_{\xi}\right\|_{\infty}^{2}\right)\left\|\gamma_{y}\right\|_{\infty}^{2}+\left\|\gamma_{\xi}^{*} \gamma_{y}\right\|_{\infty}^{2}\right] } \\
& +\left[\left(1-\left\|\gamma_{\xi}\right\|_{\infty}^{2}\right)\left(1-\left\|h_{y}\right\|_{\infty}^{2}\right)+\left\|h_{\xi}\right\|_{\infty}^{2}\left\|\gamma_{y}\right\|_{\infty}^{2}-2\left\|\gamma_{\xi}^{*} \gamma_{y}\right\|_{\infty}\left\|h_{\xi}^{*} h_{y}\right\|_{\infty}\right] \nu_{2} \\
=- & {\left[\left\|h_{\xi}\right\|_{\infty}^{2}-\left(\left\|h_{\xi}\right\|_{\infty}^{2}\left\|h_{y}\right\|_{\infty}^{2}-\left\|h_{\xi}^{*} h_{y}\right\|_{\infty}^{2}\right)\right] \nu_{2}^{2} } \\
& -\left[\left\|\gamma_{y}\right\|_{\infty}^{2}-\left(\left\|\gamma_{\xi}\right\|_{\infty}^{2}\left\|\gamma_{y}\right\|_{\infty}^{2}-\left\|\gamma_{\xi}^{*} \gamma_{y}\right\|_{\infty}^{2}\right)\right] \\
& +\left[1-\left\|\gamma_{\xi}\right\|_{\infty}^{2}-\left\|h_{y}\right\|_{\infty}^{2}+\left(\left\|\gamma_{\xi}\right\|_{\infty}\left\|h_{y}\right\|_{\infty}-\left\|\gamma_{y}\right\|_{\infty}\left\|h_{\xi}\right\|_{\infty}\right)^{2}\right. \\
& \left.+2\left(\left\|\gamma_{\xi}\right\|_{\infty}\left\|\gamma_{y}\right\|_{\infty}\left\|h_{\xi}\right\|_{\infty}\left\|h_{y}\right\|_{\infty}-\left\|\gamma_{\xi}^{*} \gamma_{y}\right\|_{\infty}\left\|h_{\xi}^{*} h_{y}\right\|_{\infty}\right)\right] \nu_{2} \\
\equiv- & c_{2} \nu_{2}^{2}-c_{0}+c_{1} \nu_{2} .
\end{aligned}
$$

Thus,

$$
c_{2} \leq\left\|h_{\xi}\right\|_{\infty}^{2}, \quad c_{0} \leq\left\|\gamma_{y}\right\|_{\infty}^{2}, \quad c_{1} \geq 1-\left\|\gamma_{\xi}\right\|_{\infty}^{2}-\left\|h_{y}\right\|_{\infty}^{2} .
$$

Hence, it suffices to require:

$$
0<-\left\|h_{\xi}\right\|_{\infty}^{2} \nu_{2}^{2}-\left\|\gamma_{y}\right\|_{\infty}^{2}+\left(1-\left\|\gamma_{\xi}\right\|_{\infty}^{2}-\left\|h_{y}\right\|_{\infty}^{2}\right) \nu_{2} \equiv f_{\gamma h}\left(\nu_{2}\right)>0,
$$

for some $\nu_{2}>0$. By (6.52), we have

$$
\Delta_{\gamma h} \triangleq\left(1-\left\|\gamma_{\xi}\right\|_{\infty}^{2}-\left\|h_{y}\right\|_{\infty}^{2}\right)^{2}-4\left\|h_{\xi}\right\|_{\infty}^{2}\left\|\gamma_{y}\right\|_{\infty}^{2}>0 .
$$


Making use of Lemma 6.3, we have

$$
\left\{f_{\gamma h}>0\right\}=\left(\mu_{-}^{\gamma h}, \mu_{+}^{\gamma h}\right),
$$

with

$$
\left\{\begin{aligned}
\mu_{-}^{\gamma h} & =\frac{2\left\|\gamma_{y}\right\|_{\infty}^{2}}{1-\left\|\gamma_{\xi}\right\|_{\infty}^{2}-\left\|h_{y}\right\|_{\infty}^{2}-\sqrt{\Delta_{\gamma h}}}, \\
\mu_{+}^{\gamma h} & = \begin{cases}\frac{1-\left\|\gamma_{\xi}\right\|_{\infty}^{2}-\left\|h_{y}\right\|_{\infty}^{2}+\sqrt{\Delta_{\gamma h}}}{2\left\|h_{\xi}\right\|_{\infty}^{2}}, & \text { if }\left\|h_{\xi}\right\|_{\infty}>0, \\
\infty, & \text { if }\left\|h_{\xi}\right\|_{\infty}=0 .\end{cases}
\end{aligned}\right.
$$

Next, to ensure (6.60), it suffices to require

$$
\left(\begin{array}{ccc}
\left(\left\|\sigma_{x}\right\|_{\infty}^{2}-\lambda_{b}^{0}\right) I & b_{y}+\sigma_{x}^{T} \sigma_{y}-\nu_{2} g_{x}^{T} & b_{z}+\sigma_{x}^{T} \sigma_{z} \\
b_{y}^{T}+\sigma_{y}^{T} \sigma_{x}-\nu_{2} g_{x} & \left(\left\|\sigma_{y}\right\|_{\infty}^{2}-\nu_{2} \lambda_{g}^{0}\right) I & \sigma_{y}^{T} \sigma_{z}-\nu_{2} g_{z} \\
b_{z}^{T}+\sigma_{z}^{T} \sigma_{x} & \sigma_{z}^{T} \sigma_{y}-\nu_{2} g_{z}^{T} & \left(\left\|\sigma_{z}\right\|_{\infty}^{2}-\nu_{2}\right) I
\end{array}\right) \ll 0 .
$$

Note that by 6.53), we can choose a $\nu_{2} \in\left(\left\|\sigma_{z}\right\|_{\infty}^{2} \vee \mu_{-}^{\gamma h}, \mu_{+}^{\gamma h}\right)$. For such a $\nu_{2}$, we can choose $\lambda_{0}>0$ large enough so that the above holds. Then we have constructed a diagonal type (III) Lyapunove operator.

To conclude this subsection, let us present the following result, which refines Proposition 6.6 when some further conditions are satisfied.

Proposition 6.7. Let $(b, \sigma, g, \gamma, h) \in \mathcal{G}^{p}$ such that

$$
\left\|\gamma_{\xi}\right\|_{\infty}=\left\|h_{y}\right\|_{\infty}=\left\|b_{z}\right\|_{\infty}=\left\|\sigma_{z}\right\|_{\infty}=\left\|g_{z}\right\|_{\infty}=0
$$

and

$$
\left\|\gamma_{y}\right\|_{\infty}^{2}\left\|h_{\xi}\right\|_{\infty}^{2}<1
$$

Let $\lambda_{b \sigma}, \lambda_{g}>0$ be defined by (6.41) and (6.27). Let

$$
\Delta \triangleq \lambda_{b \sigma}\left(\lambda_{b \sigma} \lambda_{g}^{2}-4 \lambda_{g}\left\|g_{x}\right\|_{\infty}\left\|b_{y}+\sigma_{x}^{T} \sigma_{y}\right\|_{\infty}-4\left\|g_{x}\right\|_{\infty}^{2}\left\|\sigma_{y}\right\|_{\infty}^{2}\right)>0
$$

and

$$
\rho_{-} \vee\left\|\gamma_{y}\right\|_{\infty}^{2}<\rho_{+} \wedge \frac{1}{\left\|h_{\xi}\right\|_{\infty}^{2}} \quad\left(\frac{1}{0}=\infty\right)
$$

where

$$
\left\{\begin{array}{l}
\rho_{-}=\frac{2\left(\lambda_{b \sigma}\left\|\sigma_{y}\right\|_{\infty}^{2}+\left\|b_{y}+\sigma_{x}^{T} \sigma_{y}\right\|_{\infty}^{2}\right)}{\lambda_{b \sigma} \lambda_{g}-2\left\|g_{x}\right\|_{\infty}\left\|b_{y}+\sigma_{x}^{T} \sigma_{y}\right\|_{\infty}+\sqrt{\Delta}}, \\
\rho_{+}= \begin{cases}\frac{\lambda_{b \sigma} \lambda_{g}-2\left\|g_{x}\right\|_{\infty}\left\|b_{y}+\sigma_{x}^{T} \sigma_{y}\right\|_{\infty}+\sqrt{\Delta}}{2\left\|g_{x}\right\|_{\infty}^{2}}, & \left\|g_{x}\right\|_{\infty}>0 \\
\infty, & \left\|g_{x}\right\|_{\infty}=0 .\end{cases}
\end{array}\right.
$$

Then generator $(b, \sigma, g, \gamma, h)$ admits a diagonal type (III) Lyapunov operator $P(\cdot)$.

Proof. By taking $\kappa_{1}, \kappa_{2}>0$ and $\nu_{1}=1$, we see that a diagonal type (III) Lyapunov operator exists if

$$
\left(\begin{array}{cc}
I-\nu_{2} h_{\xi}^{*} h_{\xi} & 0 \\
0 & \nu_{2} I-\gamma_{y}^{T} \gamma_{y}
\end{array}\right) \gg 0
$$


and

$$
\left(\begin{array}{ccc}
b_{x}+b_{x}^{T}+\sigma_{x}^{T} \sigma_{x} & b_{y}+\sigma_{x}^{T} \sigma_{y}-\nu_{2} g_{x}^{T} & 0 \\
b_{y}^{T}+\sigma_{y}^{T} \sigma_{x}-\nu_{2} g_{x} & \sigma_{y}^{T} \sigma_{y}-\nu_{2}\left(g_{y}+g_{y}^{T}\right) & 0 \\
0 & 0 & -\nu_{2} I
\end{array}\right) \ll 0,
$$

for some $\nu_{2}>0$.

Note that (6.67) is implied by the following:

$$
1-\nu_{2}\left\|h_{\xi}\right\|_{\infty}^{2}>0, \quad \nu_{2}-\left\|\gamma_{y}\right\|_{\infty}^{2}>0
$$

for some $\nu_{2}>0$, which is equivalent to the following:

$$
\begin{aligned}
0 & <\left(1-\nu_{2}\left\|h_{\xi}\right\|_{\infty}^{2}\right)\left(\nu_{2}-\left\|\gamma_{y}\right\|_{\infty}^{2}\right) \\
& =-\left\|h_{\xi}\right\|_{\infty}^{2} \nu_{2}^{2}+\left(1-\left\|h_{\xi}\right\|_{\infty}^{2}\left\|\gamma_{y}\right\|_{\infty}^{2}\right) \nu_{2}-\left\|\gamma_{y}\right\|_{\infty}^{2} \equiv f_{\gamma h}\left(\nu_{2}\right),
\end{aligned}
$$

for some $\nu_{2}>\left\|\gamma_{y}\right\|_{\infty}^{2}$. Clearly, the above can be guaranteed by (6.63) with

$$
\left\{\nu_{2}>\left\|\gamma_{y}\right\|_{\infty}^{2} \mid f_{\gamma h}\left(\nu_{2}\right)>0\right\}=\left(\left\|\gamma_{y}\right\|_{\infty}^{2}, \frac{1}{\left\|h_{\xi}\right\|_{\infty}^{2}}\right), \quad\left(\frac{1}{0} \triangleq \infty\right) .
$$

Next, we look at (6.68). To ensure that, it suffices to require

$$
\left(\begin{array}{cc}
b_{x}+b_{x}^{T}+\sigma_{x}^{T} \sigma_{x} & b_{y}+\sigma_{x}^{T} \sigma_{y}-\nu_{2} g_{x}^{T} \\
b_{y}^{T}+\sigma_{y}^{T} \sigma_{x}-\rho g_{x} & \sigma_{y}^{T} \sigma_{y}-\nu_{2}\left(g_{y}+g_{y}^{T}\right)
\end{array}\right) \ll 0,
$$

for some $\nu_{2}>0$. Thus, we need

$$
\lambda_{b \sigma}>0, \quad \nu_{2} \lambda_{g}-\left\|\sigma_{y}\right\|_{\infty}^{2}>0,
$$

and

$$
\begin{gathered}
0<\lambda_{b \sigma}\left(\nu_{2} \lambda_{g}-\left\|\sigma_{y}\right\|_{\infty}^{2}\right)-\left(\left\|b_{y}+\sigma_{x}^{T} \sigma_{y}\right\|_{\infty}+\nu_{2}\left\|g_{x}\right\|_{\infty}\right)^{2} \\
=-\left\|g_{x}\right\|_{\infty}^{2} \nu_{2}^{2}+\left(\lambda_{b \sigma} \lambda_{g}-2\left\|g_{x}\right\|_{\infty}\left\|b_{y}+\sigma_{x}^{T} \sigma_{y}\right\|_{\infty}\right) \nu_{2} \\
-\left(\lambda_{b \sigma}\left\|\sigma_{y}\right\|_{\infty}^{2}+\left\|b_{y}+\sigma_{x}^{T} \sigma_{y}\right\|_{\infty}^{2}\right) \equiv f\left(\nu_{2}\right) .
\end{gathered}
$$

Note that (6.64) implies

$$
\lambda_{b \sigma} \lambda_{g}-2\left\|g_{x}\right\|_{\infty}\left\|b_{y}+\sigma_{x}^{T} \sigma_{y}\right\|_{\infty}>0
$$

and

$$
\begin{aligned}
& \left(\lambda_{b \sigma} \lambda_{g}-2\left\|g_{x}\right\|_{\infty}\left\|b_{y}+\sigma_{x}^{T} \sigma_{y}\right\|_{\infty}\right)^{2}-4\left\|g_{x}\right\|_{\infty}^{2}\left(\lambda_{b \sigma}\left\|\sigma_{y}\right\|_{\infty}^{2}+\left\|b_{y}+\sigma_{x}^{T} \sigma_{y}\right\|_{\infty}^{2}\right) \\
& =\lambda_{b \sigma}^{2} \lambda_{g}^{2}-4 \lambda_{b \sigma} \lambda_{g}\left\|g_{x}\right\|_{\infty}\left\|b_{y}+\sigma_{x}^{T} \sigma_{y}\right\|_{\infty}-4 \lambda_{b \sigma}\left\|g_{x}\right\|_{\infty}^{2}\left\|\sigma_{y}\right\|_{\infty}^{2} \\
& =\lambda_{b \sigma}\left(\lambda_{b \sigma} \lambda_{g}^{2}-4 \lambda_{g}\left\|g_{x}\right\|_{\infty}\left\|b_{y}+\sigma_{x}^{T} \sigma_{y}\right\|_{\infty}-4\left\|g_{x}\right\|_{\infty}^{2}\left\|\sigma_{y}\right\|_{\infty}^{2}\right) \equiv \Delta>0 .
\end{aligned}
$$

Then by Lemma 6.3, we obtain the existence of a diagonal type (III) Lyapunov operator.

We see that the type (III) Lyapunov operator under the conditions of Proposition 6.7 is essentially of the form $\left(\begin{array}{cc}I & 0 \\ 0 & -\nu_{2} I\end{array}\right)$. 


\section{AN EXAMPLE AND CONCLUDING REMARKS}

In this section, we would like to look at the following FBSDE:

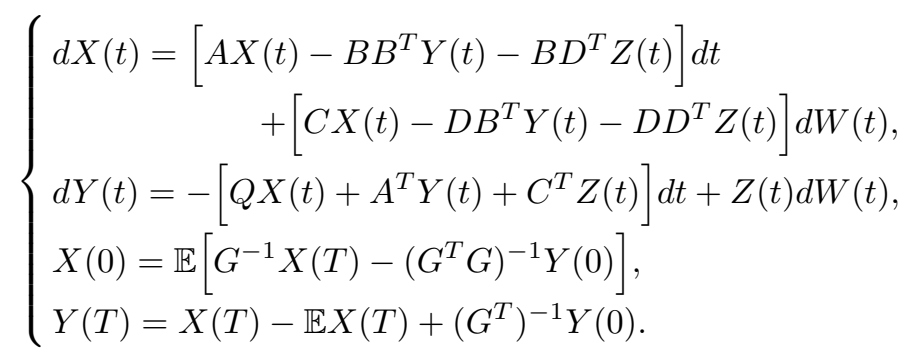

This is a special case of (2.3) for which

$$
b(t, x)=A x, \quad \sigma(t, x)=C x, \quad Q(t, x)=\frac{1}{2}\langle Q x, x\rangle .
$$

For simplicity, we assume that all the coefficients are constants and $m=n$. We have the following result.

Proposition 7.1. Suppose

$$
Q \geq 0, \quad e^{\lambda T}\left|\left(G G^{T}\right)^{-1}\right|<1, \quad e^{\lambda T}\left|\left(G^{T} G\right)^{-1}\right|<1,
$$

where

$$
\lambda=\max \Lambda\left(A+A^{T}+C^{T} C\right) .
$$

Then FBSDE (7.1) admits a unique adapted solution.

Proof. For this case, we have

$$
\gamma(\xi, y)=\mathbb{E}\left[G^{-1} \xi\right]-\left(G^{T} G\right)^{-1} y, \quad h(\xi, y)=\xi-\mathbb{E} \xi+\left(G^{T}\right)^{-1} y .
$$

Thus,

$$
\left\{\begin{array}{l}
\gamma_{\xi}=\mathbb{E} G^{-1}, \quad \gamma_{y}=-\left(G^{T} G\right)^{-1} \\
h_{\xi}=I_{\mathcal{X}^{2}}-\mathbb{E}, \quad h_{y}=I_{\mathcal{X}^{2}}\left(G^{T}\right)^{-1}
\end{array}\right.
$$

leading to

$$
\left\{\begin{array}{l}
\gamma_{\xi}^{*}=I_{\mathcal{X}^{2}}\left(G^{T}\right)^{-1}=h_{y}, \quad \gamma_{y}^{T}=-\left(G^{T} G\right)^{-1}=\gamma_{y}, \\
h_{\xi}^{*}=I_{\mathcal{X}^{2}}-\mathbb{E}=h_{\xi}, \quad h_{y}^{*}=\mathbb{E} G^{-1}=\gamma_{\xi} .
\end{array}\right.
$$

Hence, taking $\Psi=I$, we have

$$
\left(\begin{array}{cc}
\Psi^{T} h_{\xi}+h_{\xi}^{*} \Psi & \Psi^{T} h_{y}-\gamma_{\xi}^{*} \Psi^{T} \\
h_{y}^{*} \Psi-\Psi \gamma_{\xi} & -\Psi \gamma_{y}-\gamma_{y}^{T} \Psi^{T}
\end{array}\right)=\left(\begin{array}{cc}
2\left(I_{\mathcal{X}^{2}}-\mathbb{E}\right) & 0 \\
0 & 2\left(G^{T} G\right)^{-1}
\end{array}\right) \geq 0
$$

and

$$
\left(\begin{array}{ccc}
\Psi^{T} g_{x}+g_{x}^{T} \Psi & b_{x}^{T} \Psi^{T}+\Psi^{T} g_{y} & \Psi^{T} g_{z}+\sigma_{x}^{T} \Psi^{T} \\
\Psi b_{x}+g_{y}^{T} \Psi & \Psi b_{y}+b_{y}^{T} \Psi^{T} & \Psi b_{z}+\sigma_{y}^{T} \Psi^{T} \\
g_{z}^{T} \Psi+\Psi \sigma_{x} & b_{z}^{T} \Psi^{T}+\Psi \sigma_{y} & \sigma_{z}^{T} \Psi^{T}+\Psi \sigma_{z}
\end{array}\right)=\left(\begin{array}{ccc}
-2 Q & 0 & 0 \\
0 & -2 B B^{T} & -2 B D^{T} \\
0 & -2 D B^{T} & -2 D D^{T}
\end{array}\right) \leq 0 .
$$

Consequently, (5.12) becomes

$$
\left(\begin{array}{cc}
\nu_{1}\left[I-e^{\kappa_{1} T}\left(G G^{T}\right)^{-1} \mathbb{E}\right]+\left(2-\nu_{2} e^{\kappa_{2} T}\right)(I-\mathbb{E}) & \nu_{1} e^{\kappa_{1} T}\left(G^{T} G G^{T}\right)^{-1} \\
\nu_{1} e^{\kappa_{1} T}\left(G G^{T} G\right)^{-1} & \nu_{2}\left[I-e^{\kappa_{2} T}\left(G^{T} G\right)^{-1} \mathbb{E}\right]+\left[2 I-\nu_{1} e^{\kappa_{1} T}\left(G^{T} G\right)^{-1}\right]\left(G^{T} G\right)^{-1}
\end{array}\right) \gg 0,
$$


which is true if

$$
\begin{cases}e^{\kappa_{1} T}\left|\left(G G^{T}\right)^{-1}\right|<1, & \nu_{2} e^{\kappa_{2} T} \leq 2 \\ e^{\kappa_{2} T}\left|\left(G^{T} G\right)^{-1}\right| \leq 1, & \nu_{1} e^{\kappa_{1} T}\left|\left(G^{T} G\right)^{-1}\right| \leq 2\end{cases}
$$

and

$$
\left(\begin{array}{cc}
\nu_{1}\left[I-\left(G G^{T}\right)^{-1} \mathbb{E}\right]+\left(2-\nu_{2}\right)(I-\mathbb{E}) & \nu_{1}\left(G^{T} G G^{T}\right)^{-1} \\
\nu_{1}\left(G G^{T} G\right)^{-1} & \nu_{2}\left[I-\left(G^{T} G\right)^{-1} \mathbb{E}\right]-\nu_{1}\left(G^{T} G\right)^{-2}+2\left(G^{T} G\right)^{-1}
\end{array}\right) \gg 0,
$$

which is implied by

$$
\left(\begin{array}{cc}
\nu_{1}\left[I-\left(G G^{T}\right)^{-1} \mathbb{E}\right] & \nu_{1}\left(G^{T} G G^{T}\right)^{-1} \\
\nu_{1}\left(G G^{T} G\right)^{-1} & {\left[2-\nu_{1}\left(G^{T} G\right)^{-1}\right]\left(G^{T} G\right)^{-1}}
\end{array}\right) \gg 0 .
$$

This is true if $\nu_{1}>0$ is small enough.

On the other hand, (5.13) becomes (take $\alpha>0$ )

$$
\begin{aligned}
& \left(\begin{array}{ccc}
-\nu_{1} e^{\kappa_{1}(T-t)}\left[\left(\kappa_{1}-\alpha\right) I-A-A^{T}-C^{T} C\right] & \nu_{2} e^{\kappa_{2} t} Q & 0 \\
\nu_{2} e^{\kappa_{2} t} Q & -\nu_{2} e^{\kappa_{2} t}\left(\kappa_{2} I-A-A^{T}\right) & \nu_{2} e^{\kappa_{2} t} C^{T} \\
0 & \nu_{2} e^{\kappa_{2} t} C & -\nu_{2} e^{\kappa_{2} t} I
\end{array}\right) \\
& +\left(\begin{array}{ccc}
-\alpha \nu_{1} e^{\kappa_{1}(T-t)} I-2 Q & -\nu_{1} e^{\kappa_{1}(T-t)}\left(B+C^{T} D\right) B^{T} & -\nu_{1} e^{\kappa_{1}(T-t)}\left(B+C^{T} D\right) D^{T} \\
-\nu_{1} e^{\kappa_{1}(T-t)} B\left(B^{T}+D^{T} C\right) & -B\left[2 I-\nu_{1} e^{\kappa_{1}(T-t)} D^{T} D\right] B^{T} & -B\left[2 I-\nu_{1} e^{\kappa_{1}(T-t)} D^{T} D\right] D^{T} \\
-\nu_{1} e^{\kappa_{1}(T-t)} D\left(B^{T}+D^{T} C\right) & -D\left[2 I-\nu_{1} e^{\kappa_{1}(T-t)} D^{T} D\right] B^{T} & -D\left[2 I-\nu_{1} e^{\kappa_{1}(T-t)} D^{T} D\right] D^{T}
\end{array}\right) \ll 0 .
\end{aligned}
$$

We require

$$
\left(\begin{array}{ccc}
-\alpha \nu_{1} e^{\kappa_{1}(T-t)} I-2 Q & -\nu_{1} e^{\kappa_{1}(T-t)}\left(B+C^{T} D\right) B^{T} & -\nu_{1} e^{\kappa_{1}(T-t)}\left(B+C^{T} D\right) D^{T} \\
-\nu_{1} e^{\kappa_{1}(T-t)} B\left(B^{T}+D^{T} C\right) & -B\left[2 I-\nu_{1} e^{\kappa_{1}(T-t)} D^{T} D\right] B^{T} & -B\left[2 I-\nu_{1} e^{\kappa_{1}(T-t)} D^{T} D\right] D^{T} \\
-\nu_{1} e^{\kappa_{1}(T-t)} D\left(B^{T}+D^{T} C\right) & -D\left[2 I-\nu_{1} e^{\kappa_{1}(T-t)} D^{T} D\right] B^{T} & -D\left[2 I-\nu_{1} e^{\kappa_{1}(T-t)} D^{T} D\right] D^{T}
\end{array}\right) \leq 0,
$$

which is implied by

$$
\left(\begin{array}{ccc}
-\alpha \nu_{1} e^{\kappa_{1}(T-t)} I & -\nu_{1} e^{\kappa_{1}(T-t)}\left(B+C^{T} D\right) B^{T} & -\nu_{1} e^{\kappa_{1}(T-t)}\left(B+C^{T} D\right) D^{T} \\
-\nu_{1} e^{\kappa_{1}(T-t)} B\left(B^{T}+D^{T} C\right) & -B\left[2 I-\nu_{1} e^{\kappa_{1}(T-t)} D^{T} D\right] B^{T} & -B\left[2 I-\nu_{1} e^{\kappa_{1}(T-t)} D^{T} D\right] D^{T} \\
-\nu_{1} e^{\kappa_{1}(T-t)} D\left(B^{T}+D^{T} C\right) & -D\left[2 I-\nu_{1} e^{\kappa_{1}(T-t)} D^{T} D\right] B^{T} & -D\left[2 I-\nu_{1} e^{\kappa_{1}(T-t)} D^{T} D\right] D^{T}
\end{array}\right) \leq 0 .
$$

This is equivalent to the following: $\alpha>0$, and

$$
\begin{aligned}
& \left(\begin{array}{ll}
-B\left[2 I-\nu_{1} e^{\kappa_{1}(T-t)} D^{T} D\right] B^{T} & -B\left[2 I-\nu_{1} e^{\kappa_{1}(T-t)} D^{T} D\right] D^{T} \\
-D\left[2 I-\nu_{1} e^{\kappa_{1}(T-t)} D^{T} D\right] B^{T} & -D\left[2 I-\nu_{1} e^{\kappa_{1}(T-t)} D^{T} D\right] D^{T}
\end{array}\right) \\
& +\frac{\nu_{1} e^{\kappa_{1}(T-t)}}{\alpha}\left(\begin{array}{l}
B\left(B^{T}+D^{T} C\right) \\
D\left(B^{T}+D^{T} C\right)
\end{array}\right)\left(\left(B+C^{T} D\right) B^{T},\left(B+C^{T} D\right) D^{T}\right) \\
& =-\left(\begin{array}{c}
B \\
D
\end{array}\right)\left\{2 I-\nu_{1} e^{\kappa_{1}(T-t)}\left[D^{T} D+\frac{1}{\alpha}\left(B+C^{T} D\right)^{T}\left(B+C^{T} D\right)\right]\right\}\left(\begin{array}{c}
B \\
D
\end{array}\right)^{T} \leq 0 .
\end{aligned}
$$

Hence, for any given $\alpha, \kappa_{1}>0$, by choosing $\nu_{1}>0$ small enough, we can achieve the above. Next, we require

$$
\left(\begin{array}{ccc}
-\nu_{1} e^{\kappa_{1}(T-t)}\left[\left(\kappa_{1}-\alpha\right) I-A-A^{T}-C^{T} C\right] & \nu_{2} e^{\kappa_{2} t} Q & 0 \\
\nu_{2} e^{\kappa_{2} t} Q & -\nu_{2} e^{\kappa_{2} t}\left(\kappa_{2} I-A-A^{T}\right) & \nu_{2} e^{\kappa_{2} t} C^{T} \\
0 & \nu_{2} e^{\kappa_{2} t} C & -\nu_{2} e^{\kappa_{2} t} I
\end{array}\right) \ll 0,
$$

which is equivalent to the following:

$$
\left(\begin{array}{ccc}
-\frac{\nu_{1} e^{\kappa_{1}(T-t)}}{\nu_{2} e^{\kappa_{2} t}}\left[\left(\kappa_{1}-\alpha\right) I-A-A^{T}-C^{T} C\right] & Q & 0 \\
Q & -\kappa_{2} I-A-A^{T} & C^{T} \\
0 & C & -I
\end{array}\right) \ll 0 .
$$


This is equivalent to the following:

$$
\left(\kappa_{1}-\alpha\right) I-A-A^{T}-C^{T} C \gg 0
$$

and

$$
\left(\begin{array}{cc}
-\left(\kappa_{2} I-A-A^{T}\right)+\frac{\nu_{2} \kappa^{\kappa_{2} t}}{\nu_{1} e^{\kappa_{1}(T-t)}} Q\left[\left(\kappa_{1}-\alpha\right) I-A-A^{T}-C^{T} C\right]^{-1} Q & C^{T} \\
C & -I
\end{array}\right) \ll 0,
$$

which is further equivalent to the following:

$$
-\left(\kappa_{2} I-A-A^{T}\right)+\frac{\nu_{2} e^{\kappa_{2} t}}{\nu_{1} e^{\kappa_{1}(T-t)}} Q\left[\left(\kappa_{1}-\alpha\right) I-A-A^{T}-C^{T} C\right]^{-1} Q+C^{T} C \ll 0 .
$$

This is implied by

$$
-\left(\kappa_{2} I-A-A^{T}-C^{T} C\right)+\frac{\nu_{2} e^{\kappa_{2} T}}{\nu_{1}} Q\left[\left(\kappa_{1}-\alpha\right) I-A-A^{T}-C^{T} C\right]^{-1} Q \ll 0 .
$$

Now, we first choose $\kappa_{1}>\alpha>0$ such that

$$
\kappa_{1}-\alpha>\max \Lambda\left(A+A^{T}+C^{T} C\right) .
$$

Then choose $\nu_{1}>0$ small so that

$$
\nu_{1} e^{\kappa_{1} T}\left|D^{T} D+\frac{1}{\alpha}\left(B+C^{T} D\right)^{T}\left(B+C^{T} D\right)\right| \leq 2 .
$$

Next, choose $\kappa_{2}>0$ such that

$$
\kappa_{2}>\max \Lambda\left(A+A^{T}+C^{T} C\right)+\varepsilon\left|Q\left[\left(\kappa_{1}-\alpha\right) I-A-A^{T}-C^{T} C\right]^{-1} Q\right| .
$$

Finally, we choose

$$
\nu_{2}=\varepsilon \nu_{1} e^{-\kappa_{2} T} .
$$

Note that for $\varepsilon, \alpha>0$ small enough, we can have $\kappa_{1}, \kappa_{2}>\lambda$ such that (7.5) holds. Hence, the generator of FBSDE (7.1) admits a type (III) Lyapunov operator $P(\cdot)$ of the form (5.14) with $\Psi=I$. Hence, (7.1) admits a unique adapted solution.

Note that the left hand side of (7.3) is merely positive semi-definite and the left hand side of (7.4) is merely negative semi-definite. Hence, $P=\left(\begin{array}{ll}0 & I \\ I & 0\end{array}\right)$ cannot serve as a Lyapunov operator for the generator. We have seen how we choose the diagonal elements to have a type (III) Lyapunov operator leading to the well-posedness of the FBSDE.

To conclude this paper, we would like to make several remarks.

(i) For simplicity of presentation, we only considered the case of one-dimensional Brownian motion. The higher-dimensional Brownian motion case should have a similar theory.

(ii) We did not present examples for which either type (I) or type (II) Lyapunov operators exist but type (III) Lyapunov operators do not exist. We believe that such examples do exist.

(iii) We have not looked into the construction of general Lyapunov operators $P(t)=\left(\begin{array}{cc}P_{1}(t) & \Psi(t)^{T} \\ \Psi(t) & P_{2}(t)\end{array}\right)$. In particular, the author believes that even the form $P(t)=\left(\begin{array}{cc}\nu_{1} e^{\kappa_{1}(T-t)} I & \Psi(t)^{T} \\ \Psi(t) & -\nu_{2} e^{\kappa_{2} t} I\end{array}\right)$ (allowing $\Psi$ to be time-varying) will lead to the well-posedness of a larger class of FBSDEs. 
(iv) It is possible to define stochastic Lyapunov operators; i.e., one may allow $P(\cdot)$ to be an $\mathbb{F}$-adapted stochastic process. Then the well-posedness of a much wider class of FBSDEs might be established. We hope to present some interesting results along this line in the future.

\section{ACKNowledgement}

The author would like to thank the anonymous referee for his/her suggested comments which led to the current improved version.

\section{ApPENDix}

Consider a controlled SDE:

$d X(t)=[b(t, X(t))+B(t) u(t)] d t+[\sigma(t, X(t))+D(t) u(t)] d W(t), \quad t \in[0, T]$,

with the following cost functional:

$$
J(u(\cdot))=\mathbb{E}\left\{\int_{0}^{T}\left[Q(t, X(t))+\frac{1}{2}|u(t)|^{2}\right] d t+\frac{1}{2}|X(T)-G X(0)|^{2}\right\} .
$$

Suppose $(\bar{X}(\cdot), \bar{u}(\cdot))$ is an optimal pair. Then take any control $u(\cdot)$, initial state $X_{0} \in \mathbb{R}^{n}$, and $\varepsilon>0$. Letting $X^{\varepsilon}(\cdot)$ be the state corresponding to $u^{\varepsilon}(\cdot)=\bar{u}(\cdot)+\varepsilon u(\cdot)$ with the initial state $X_{0}+\varepsilon \bar{X}(0)$, we have

$$
\lim _{\varepsilon \rightarrow 0} \frac{X^{\varepsilon}(t)-\bar{X}(t)}{\varepsilon}=X(t), \quad t \in[0, T]
$$

with

$$
\left\{\begin{array}{l}
d X(t)=\left[b_{x}(t, \bar{X}(t)) X(t)+B(t) u(t)\right] d t+\left[\sigma_{x}(t, \bar{X}(t)) X(t)+D(t) u(t)\right] d W(t), \\
X(0)=X_{0},
\end{array}\right.
$$

and

$$
\begin{aligned}
0 \leq & \lim _{\varepsilon \rightarrow 0} \frac{J\left(u^{\varepsilon}(\cdot)\right)-J(\bar{u}(\cdot))}{\varepsilon}=\mathbb{E}\left\{\int_{0}^{T}\left[\left\langle Q_{x}(t, \bar{X}(t)), X(t)\right\rangle+\langle\bar{u}(t), u(t)\rangle\right] d t\right. \\
& \left.+\langle\bar{X}(T)-G \bar{X}(0), X(T)\rangle-\left\langle G^{T}[\bar{X}(T)-G \bar{X}(0)], X(0)\right\rangle\right\} .
\end{aligned}
$$

Now, introduce BSDE:

$$
d Y(t)=\varphi(t) d t+Z(t) d W(t)
$$

with $\varphi(\cdot)$ undetermined. By Itô's formula, we have (suppressing $t$ and $\bar{X}$ )

$$
\begin{gathered}
d\langle X, Y\rangle=\left[\left\langle b_{x} X+B u, Y\right\rangle+\langle X, \varphi\rangle+\left\langle\sigma_{x} X+D u, Z\right\rangle\right] d t \\
+\left[\left\langle\sigma_{x} X+D u, Y\right\rangle+\langle X, Z\rangle\right] d W
\end{gathered}
$$

Hence,

$$
\begin{aligned}
& \mathbb{E}[\langle X(T), Y(T)\rangle-\langle X(0), Y(0)\rangle] \\
& =\mathbb{E} \int_{0}^{T}\left[\left\langle b_{x} X+B u, Y\right\rangle+\langle X, \varphi\rangle+\left\langle\sigma_{x} X+D u, Z\right\rangle\right] d t .
\end{aligned}
$$


Consequently,

$$
\begin{aligned}
& 0 \leq \mathbb{E}\left\{\int_{0}^{T}\left[\left\langle Q_{x}, X\right\rangle+\langle\bar{u}, u\rangle+\left\langle b_{x} X+B u, Y\right\rangle+\langle X, \varphi\rangle+\left\langle\sigma_{x} X+D u, Z\right\rangle\right] d t\right. \\
& +\langle\bar{X}(T)-G \bar{X}(0), X(T)\rangle-\left\langle G^{T}[\bar{X}(T)-G \bar{X}(0)], X(0)\right\rangle \\
& -(\langle X(T), Y(T)\rangle-\langle X(0), Y(0)\rangle)\} \\
& =\mathbb{E}\left\{\int_{0}^{T}\left[\left\langle Q_{x}+b_{x}^{T} Y+\sigma_{x}^{T} Z+\varphi, X\right\rangle+\left\langle\bar{u}+B^{T} Y+D^{T} Z, u\right\rangle\right] d t\right. \\
& +\langle\bar{X}(T)-G \bar{X}(0)-Y(T), X(T)\rangle \\
& \left.-\left\langle G^{T}[\bar{X}(T)-G \bar{X}(0)]-Y(0), X(0)\right\rangle\right\} .
\end{aligned}
$$

Hence, we must have

$$
\left\{\begin{array}{l}
Y(0)=\mathbb{E} G^{T}[\bar{X}(T)-G \bar{X}(0)], \quad Y(T)=\bar{X}(T)-G \bar{X}(0), \\
\varphi=-b_{x}^{T} Y-\sigma_{x}^{T} Z-Q_{x}, \quad \bar{u}=-B^{T} Y-D^{T} Z
\end{array}\right.
$$

Thus,

$$
\bar{X}(0)=\left(G^{T} G\right)^{-1}\left[\mathbb{E} G^{T} \bar{X}(T)-Y(0)\right]=\mathbb{E}\left[G^{-1} \bar{X}(T)-\left(G^{T} G\right)^{-1} Y(0)\right]
$$

and

$$
\begin{aligned}
Y(T) & =\bar{X}(T)-G \mathbb{E}\left[G^{-1} \bar{X}(T)-\left(G^{T} G\right)^{-1} Y(0)\right] \\
& =\bar{X}(T)-\mathbb{E} \bar{X}(T)+\left(G^{T}\right)^{-1} Y(0) .
\end{aligned}
$$

Therefore, the optimality system takes the following form (dropping bars):

$$
\left\{\begin{aligned}
& d X(t)= {\left[b(t, X(t))-B(t) B(t)^{T} Y(t)-B(t) D(t)^{T} Z(t)\right] d t } \\
&+\left[\sigma(t, X(t))-D(t) B(t)^{T} Y(t)-D(t) D(t)^{T} Z(t)\right] d W(t), \\
& d Y(t)=-\left[b_{x}(t, X(t))^{T} Y(t)+\sigma_{x}(t, X(t))^{T} Z(t)+Q_{x}(t, X(t))\right] d t \\
& X(0)=\mathbb{E}\left[G^{-1} \bar{X}(T)-\left(G^{T} G\right)^{-1} Y(0)\right] \\
& Y(T)= X(T)-\mathbb{E} X(T)+\left(G^{T}\right)^{-1} Y(0) .
\end{aligned}\right.
$$

\section{REFERENCES}

1. M. Allais, Le comportement de l'homme rationnel devant le risque: Critique des postulats et axiomes de l'école Américaine, Econometrica, 21 (1953), 503-546. MR0058952 (15:455c)

2. F. Antonelli, Backward-forward stochastic differential equations, Ann. Appl. Probab., 3 (1993), 777-793. MR.1233625 (95a:60079)

3. F. Antonelli, E. Barucci, and M. E. Mancino, Asset pricing with a forward-backward stochastic differential utility, Economics Letters, 72 (2001), 151-157. MR.1840721 (2002c:91052)

4. L. D. Berkovitz, Optimal Control Theory, Springer-Verlag, New York, 1974. MR0372707 $(51: 8914)$

5. Z. Chen and L. Epstein, Ambiguity, risk, and asset returns in continuous time. Econometrica 70 (2002), 1403-1443. MR1929974 (2003i:91052)

6. C. De Coster and P. Habets, Two-Point Boundary Value Problems: Lower and Upper Solutions, Elsevier, Amsterdam, 2006. MR.2225284 (2007k:34001)

7. F. Delarue, On the existence and uniqueness of solutions to FBSDEs in a non-degenerate case, Stochastic Process. Appl., 99 (2002), 209-286. MR.1901154 (2003c:60108)

8. F. Delarue and G. Guatteri, Weak existence and uniqueness for forward-backward SDEs, Stochastic Process. Appl., 116 (2006), 1712-1742. MR2307056 (2008b:60125) 
9. D. Duffie and L. Epstein, Stochastic differential utility, Econometrica, 60 (1992), 353-394. MR 1162620 (93e:90020)

10. N. El Karoui, S. Peng, and M.-C. Quenez, Backward stochastic differential equations in finance, Mathematical Finance, 7 (1997), 1-71. MR1434407 (98d:90030)

11. D. Ellsberg, Risk, Ambiguity and the Savage Axioms, Quarterly Journal of Economics, 75 (1961), 643-669.

12. M. Fuhrman and G. Tessitore, Existence of optimal stochastic controls and global solutions of forward-backward stochastic differential equations, SIAM J. Control Optim., 43 (2004), 813-830. MR2114377 (2005i:93091)

13. Y. Hu, On the solution of forward-backward SDEs with monotone and continuous coefficients, Nonlinear Anal., 42 (2000), 1-12. MR1769248 (2001h:60109)

14. Y. Hu and S. Peng, Solution of forward-backward stochastic differential equations, Probab. Theory Related Fields, 103 (1995), 273-283. MR1355060 (96j:60102)

15. Y. Hu and J. Yong, Forward-backward stochastic differential equations with nonsmooth coefficients, Stoch. Proc. Appl., 87 (2000), 93-106. MR1751166 (2001c:60096)

16. A. Lazrak, Generalized stochastic differential utility and preference for information. Ann. Appl. Probab., 14 (2004), 2149-2175. MR2100387(2005i:60110)

17. A. Lazrak and M.-C. Quenez, A generalized stochastic differential utility, Math. Oper. Res., 28 (2003), 154-180. MR1961272 (2004c:60176)

18. J. Ma, P. Protter, and J. Yong, Solving forward-backward stochastic differential equations explicitly - a four step scheme, Probab. Theory Related Fields, 98 (1994), 339-359. MR:1262970 (94m:60118)

19. J. Ma and J. Yong, Solvability of forward-backward SDEs and the nodal set of HamiltonJacobi-Bellman equations, Chinese Ann. Math. Ser. B, 16 (1995), 279-298. MR.1370779 (96i:35144)

20. J. Ma and J. Yong, Forward-Backward Stochastic Differential Equations and Their Applications, Springer-Verlag, Berlin, 1999. MR 1704232 (2000k:60118)

21. E. Pardoux and S. Tang, Forward-backward stochastic differential equations and quasilinear parabolic PDEs, Probab. Theory Related Fields, 114 (1999), 123-150. MR1701517 (2000f:60088)

22. S. Peng, Backward SDE and related g-expectation, Backward stochastic differential equations (Paris, 1995-1996), 141-159, Pitman Res. Notes Math. Ser., 364, Longman, Harlow, 1997. MR 1752680

23. S. Peng and Z. Wu, Fully coupled forward-backward stochastic differential equations and applications to optimal control, SIAM J. Control Optim., 37 (1999), 825-843. MR1675098 (99m:49043)

24. M. Ronto and A. M. Samoilenko, Numerical-Analytic Methods in the Theory of BoundaryValue Problems, World Scientific, Singapore, 2000. MR.1781545 (2001h:34021)

25. M. Schroder and C. Skiadas, Optimal consumption and portfolio selection with stochastic differential utility, J. Economic Theory, 89 (1999), 68-126. MR1724377 (2001c:91040)

26. M. Schroder and C. Skiadas, Optimal lifetime consumption-portfolio strategies under trading constraints and generalized recursive preferences, Stoch. Proc. Appl., 108 (2003), 155-202. MR2016971 (2004i:91097)

27. J. von Neumann and O. Morgenstern, Theory of Games and Economic Behavior, Princeton Univ. Press, Princeton, New Jersey, 1944. MR0011937(6:235k)

28. J. Yong, Finding adapted solutions of forward-backward stochastic differential equations: method of continuation, Probab. Theory Related Fields, 107 (1997), 537-572. MR 1440146 (98e:60100)

29. J. Yong, A linear quadratic optimal control problem with generalized expectation, Stoch. Anal. Appl., to appear.

30. J. Yong and X. Y. Zhou, Stochastic Controls: Hamiltonian Systems and HJB Equations, Springer-Verlag, New York, 1999. MR 1696772(2001d:93004)

31. J. Zhang, The well posedness of FBSDEs, Discrete Contin. Dyn. Syst. B6 (2006), 927-940. MR 2223916 (2007h:60058)

Department of Mathematics, University of Central Florida, Orlando, Florida 32816

E-mail address: jyong@mail.ucf.edu 